\title{
X-ray Absorption Spectroscopy and X-ray Raman Scattering of Water and Ice; An Experimental View
}

A. Nilsson ${ }^{1,2^{*}}$, D. Nordlund ${ }^{1}$, I. Waluyo ${ }^{1}$, N. Huang ${ }^{1}$, H. Ogasawara ${ }^{1}$, S. Kaya ${ }^{1}$, U. Bergmann $^{1}$, L.-Å. Näslund ${ }^{1}$, H. Öström ${ }^{2}$, Ph. Wernet ${ }^{3}$, K. J. Andersson ${ }^{1,2}$, T. Schiros $^{1,2}$ and L. G. M. Pettersson ${ }^{2}$

${ }^{1}$ Stanford Synchrotron Radiation Lightsource, SLAC National Accelerator Laboratory, Menlo Park, CA 94025, USA

${ }^{2}$ FYSIKUM, Stockholm University, AlbaNova University Center, S-106 91 Stockholm, Sweden ${ }^{3}$ Helmholtz-Zentrum Berlin, Albert-Einstein-Str. 15, D-12489 Berlin, Germany

\begin{abstract}
Here we present a review of x-ray absorption spectroscopy and x-ray Raman scattering with the perspective to understand the spectra of water including changes with temperature, mass of the water molecule and presence of monovalent ions. The different detection schemes are discussed and it is concluded that transmission x-ray absorption measurements, using a small area where the thickness is uniform, and x-ray Raman scattering give the most reliable spectra. Different model systems are discussed such as the surface and bulk of ice and various adsorbed monolayer structures on metal surfaces.
\end{abstract}

* Corresponding author: nilsson@slac.stanford.edu 


\section{Introduction}

Water is the key compound for our existence on this planet and it is involved in many important physical, chemical, biological and geological processes. Its thermodynamic properties such as compressibility, density variation and heat capacity show many anomalies which are extreme in the supercooled region but are also present at ambient conditions. The structure of water has been debated for over 100 years since the early work by Röntgen in 1892 (1) suggesting a two-component system and the work of Bernal and Fowler in 1933 proposing a disordered quartz-like structure (2). The most established picture of water that exists in textbooks today is in terms of a homogeneous distribution of near-tetrahedral hydrogen-bonded (H-bonded) structures.

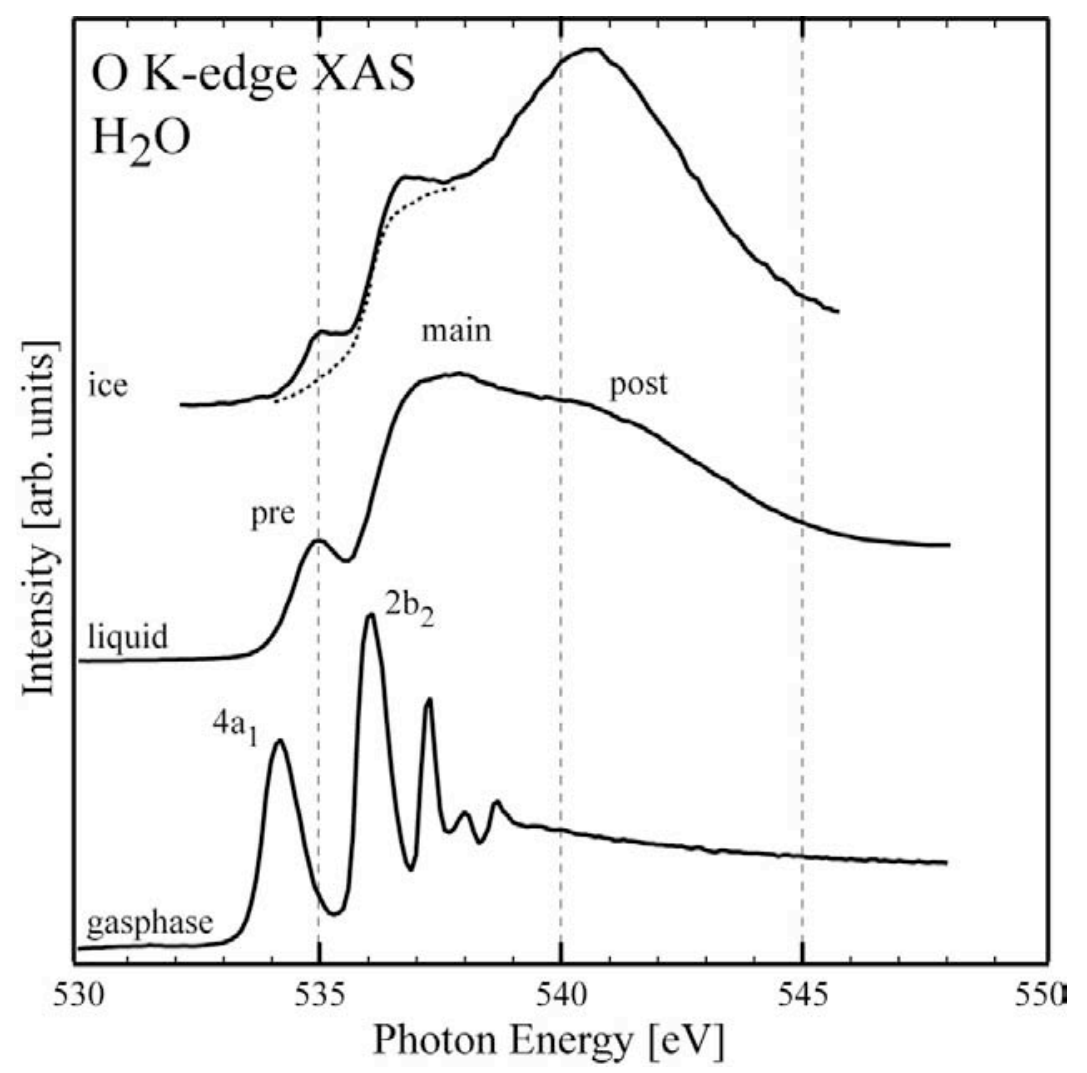

Fig.1. XAS spectra of water in the gas phase, liquid and ice $(3 ; 18 ; 30)$. The intensity above the dotted line in the ice spectrum is related to mixtures of various amorphous phases (see section V.1).

In 2004 an intense debate started after the publication of the Wernet et al. study (3) regarding the local structure of water based on X-ray Absorption Spectroscopy (XAS) and X-ray Raman Scattering (XRS) together with Density Functional Theory (DFT) spectrum calculations where it was proposed that the dominating species in the liquid is a single-donor (SD) species with one strong and one weak or broken donor H-bond. This species has also been denoted asymmetrically $\mathrm{H}$-bonded due to the difference in the H-bonding of the two $\mathrm{OH}$ groups in the water molecule (4-7). The conclusions drawn from the Wernet et al. study contradict the 
existing textbook picture of water structure and furthermore indicate that the structures obtained from molecular dynamics (MD) simulations, where the dominating species are double-donors (DD) or symmetrical structures, are in disagreement with the x-ray spectroscopic results (3). This has generated a strong controversy in the literature and many questions have been raised regarding whether we truly understand XAS applied to water and aqueous solutions $(4 ; 6 ; 8-27)$. Most of the discussion has been focused on the ability to reliably calculate theoretical XAS or XRS spectra for H-bonded systems and less on the actual experimental data. In the current special issue there is a separate article on theoretical simulations of XAS (28); here we will focus on the information contained in the experimental data and what can be learned from model experiments.

Let us first discuss the experimental data that has caused the debate. Fig. 1 shows $\mathrm{O} \mathrm{K}-$ edge XAS spectra of water in its three aggregation states indicating that the electronic transitions in the condensed forms are significantly different from those in the gas phase $(3 ; 18)$. The liquid water spectrum shows a strong pre-edge peak at $535 \mathrm{eV}$, a main-edge peak at $537.5 \mathrm{eV}$, and a post-edge peak around $541 \mathrm{eV}$ (3). The suggested interpretation in Wernet et al. is the following: The weakly coordinated $\mathrm{OH}$ in molecules with asymmetric donating $\mathrm{H}$-bonds gives rise to the strong pre-edge and main-edge features observed for liquid water, while the more strongly $\mathrm{H}$ bonded $\mathrm{OH}$ contributes to the post-edge peak $(3 ; 11 ; 18)$. In tetrahedral configurations, as in ice, both $\mathrm{OH}$ groups are coordinated with neighboring water molecules giving rise to DD species. Thereby the contribution to the intensity at the post-edge will be nearly twice as large compared to from an SD configuration. In this sense, part of the intensity of the post-edge is transferred to the pre-edge and main-edge upon breaking one donor $\mathrm{H}$-bond forming SD from DD configurations.

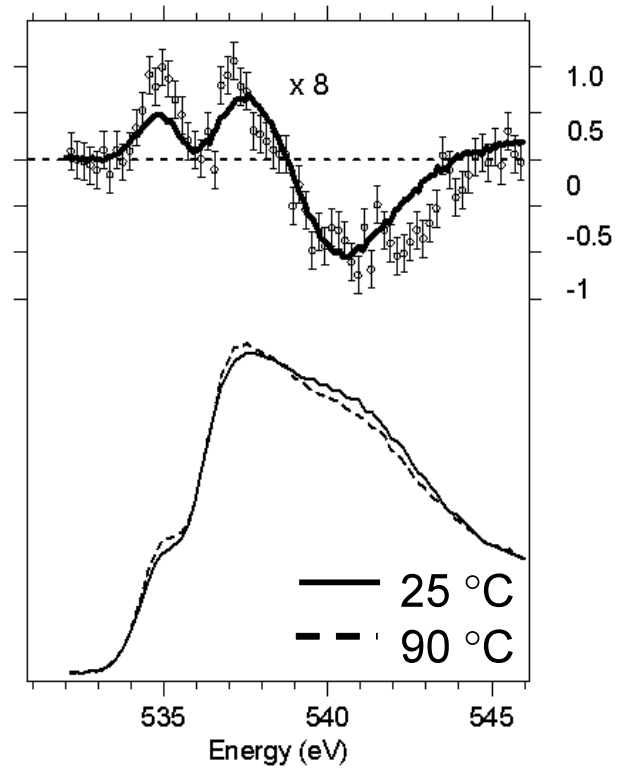

Fig. 2 XRS spectra of liquid water at $25^{\circ} \mathrm{C}$ (solid line) and $90^{\circ} \mathrm{C}$ (dashed line) (normalized to the same area) and difference spectra: $25^{\circ} \mathrm{C}$ water minus bulk ice (solid curve) and $90^{\circ} \mathrm{C}$ water minus $25^{\circ} \mathrm{C}$ water (circles with error bars, curve has been multiplied by factor 8$)(3)$.

Fig. 2 shows a comparison between XRS spectra of room temperature water $\left(25^{\circ} \mathrm{C}\right)$ and hot water $\left(90^{\circ} \mathrm{C}\right)(3)$. The changes are small compared to the changes observed between ice and the liquid indicating that room temperature and hot water have very similar local structures. 
Intensities in the pre- and main-edge regions increase while post-edge intensities decrease upon heating. Configurations with one uncoordinated or weakly $\mathrm{H}$-bonded $\mathrm{O}-\mathrm{H}$ group replace tetrahedral ones in the ice-liquid phase transition, and heating liquid water causes very similar types of spectral changes, but of $\sim 8$ times smaller magnitude. This was interpreted as conversion of DD species to SD with increasing temperature. Based on model experiments on bulk and surface of ice (see below) as well as theoretical calculations and modeling of ice spectra from the bulk and the surface it was suggested that $60-95 \%$ of the structures in the liquid are of SD character and the rest DD or non-donor (ND) species. The estimated error bar was quite large since the exact line shape of the spectra from the different species is not known.

Smith et al. studied supercooled and room temperature water using a liquid jet (24) with XAS and indicated a larger temperature dependence compared to the results in fig. 2. It was suggested, based on a temperature-dependent Boltzmann distribution analysis similar to previous $\mathrm{OH}$ stretch Raman studies (29), that the conventional homogeneous near-tetrahedral water structure model is consistent with the energetics derived from the large spectral changes (24). However, Nilsson et al. showed in a Comment (19) that the latter study is inconsistent with previously published spectral changes (3), that it appears to be victim of self-absorption (often denoted "saturation effects") resulting in experimental artifacts and that the spectral analysis was furthermore based on incorrect assumptions of spectral intensities (19).

The interpretation in Wernet et al. was also supported based on the difference between spectra of the bulk and surface of ice, where in the latter case free dangling $\mathrm{OH}$ groups generate SD species with a small post-edge and strong pre-edge $(3 ; 30)$. Upon adsorption of ammonia to saturate the dangling $\mathrm{OH}$ the surface spectrum becomes more similar to that of bulk ice $(3 ; 30)$. A subtraction between bulk and surface spectra was carried out in order to enhance the surface contribution (3). The interesting observation was the similarity of the ice surface spectrum to that of bulk water in terms of the pre-edge peak and smaller intensity of the post-edge in comparison to bulk ice. The use of the surface of ice spectrum was criticized by Smith et al. (23) who pointed out that there is a major discrepancy between the surface ice spectrum in Wernet et al. (3) and the ion-yield spectrum of Parent et al. (31) where the latter shows a much stronger pre-edge. Smith et al. (23) also raised severe questions regarding large variations of the relative spectral intensities between the pre-edge and main-edge in the experimental studies in Myneni et al. (18), Bergmann et al. (32) and Wernet et al. (3). This was also brought up in a recent study by Tse et al. applying the XRS technique to various ice forms and liquid water (26). The latter study also claimed that the pre-edge feature is more a spectroscopic core-excitonic feature and not particularly sensitive to the local H-bonding environment (26).

The core-excitonic nature of the pre-edge features was previously demonstrated through resonant Auger measurements $(20 ; 33)$ where, after excitation at the pre-edge, the electron stays localized on the water molecule for longer than 20 femtoseconds whereas it delocalizes on a timescale of around 500 attoseconds for post-edge excitations (20). This was interpreted as the pre-edge excited orbital being strongly localized on the non-H-bonded $\mathrm{OH}$ group with a larger part of the wave function in the void outside the hydrogen atom where other molecules are not present. The wave function of the post-edge excited state is mainly located along the H-bonds connecting the various molecules which allows for fast delocalization.

The localization upon excitation to the pre-edge state was also observed in resonant Xray emission spectroscopy (XES) through a spectator shift $(25)$. It has been shown $(13 ; 25)$ that the lone pair $1 b_{1}$ peak in the XES spectrum of liquid water is split in two, $1 b_{1}{ }^{\prime}$ and $1 b_{1}{ }^{\prime \prime}$, where the high energy $1 b_{1}{ }^{\prime}$ XES peak is linked to the pre-edge spectral feature and the low energy $1 b_{1}{ }^{\prime}$ 
to the post-edge in XAS (25). The temperature dependence of both XAS and XES give the same experimental observations where, in XAS, the post-edge diminishes and the pre-edge increases (3) with increasing temperature exactly in the same manner as the $1 b_{1}{ }^{\prime}$ converts to $1 b_{1}{ }^{\prime \prime}$ in XES (25). Experimentally, each spectroscopic feature in XAS can be selectively accessed by the complementary XES, since the final state of XAS corresponds to the initial or intermediate states of XES $(3 ; 14 ; 34)$. This way the $1 b_{1}$ " in XES peak is linked to the pre-edge spectral feature and the $1 b_{1}{ }^{\prime}$ to the post-edge in XAS. Since the post-edge feature is clearly enhanced in ice $(3 ; 30)$ it is consistent with that the $1 b_{1}{ }^{\prime}$ is tetrahedral-like, whereas the pre-edge peak, in XES associated to the $1 b_{1}$ ", has been discussed in terms of distorted H-bonding configurations $(3 ; 21 ; 23)$. This result has been put forward as strong evidence for the existence of two distinct structural motifs in liquid water (25). An alternative interpretation regarding the two lone pair peaks in the XES data based on ultrafast dissociation has been proposed $(13 ; 35 ; 36)$ and arguments against this interpretation are given in another article in the current special issue (37). The interpretation in terms of two structures is furthermore directly supported by a recent study using Small-Angle Xray Scattering (SAXS) measurements where the two structural species are shown to be spatially separated in the liquid providing strong independent support of the assignment (38). It is also fully consistent with an analysis of x-ray and neutron diffraction experimental data (39).

Since several of the x-ray spectroscopic results are currently strongly debated it is extremely important to be clear on what we can learn from these experiments. We will in this article focus on general experimental aspects of XAS/XRS measurements and what information various model experiments provide. We will also go through some basics regarding XAS which could be useful to readers interested in water but less familiar with x-ray spectroscopy. For a more general description of XAS we recommend the book "NEXAFS spectroscopy” by Stöhr (40).

\section{Different Measurement Techniques}

The application of XAS/XRS to water has only been developed recently due to the necessity of advanced detection systems and intense x-ray sources with a continuous spectrum, which can be produced by, e.g., a third-generation synchrotron facility. In particular the ultra high vacuum (UHV) environment required at the synchrotron beam lines has rendered oxygen $K$ edge (O $1 s$ ) XAS of water problematic due to the high vapor pressure of the liquid. The first measurement of XAS on liquid water was carried out by Yang and Kirz in transmission through a thin water film between two $\mathrm{Si}_{3} \mathrm{~N}_{4}$-windows(41) in 1980 and later Bowron et al.(42) reported the first XRS measurements in 2000. Although these experiments demonstrated the feasibility of such measurements, no information about the H-bond structure in liquid water could be derived because of the limited spectral resolution. The sharp pre-edge peak structure and broad post-edge could not be resolved at this time. The first high-resolution measurement was by Myneni et al. in 2002 (18) with a resolution of better than $0.1 \mathrm{eV}$ using fluorescence detection where all the spectral features could be resolved for the first time; the first XRS study where the pre-edge could be resolved as a shoulder was that of Bergmann et al. in 2002 (32). The sharpness of the pre-edge peak in the XAS spectrum was immediately recognized as remarkable. Most other spectroscopies applied to water, such as infrared spectroscopy and Raman spectroscopy in the $\mathrm{OH}$ stretch region, give rather structure-less spectra. However, both the Myneni et al. and Bergmann et al. studies did include some artifacts with respect to the quantitative aspects of the spectral features. This was addressed in a study by Näslund et al. in 2005 (43) where a detailed comparison of XAS/XRS measured with different detection schemes was conducted with the 
conclusion that the relative intensities of pre-, main and post edges were incorrect in the first measurements. The same issue with respect to the relative intensities was also raised by Smith et al. in 2006 (23) and by Tse et al. (26) in 2008. There are in particular two issues in XAS/XRS experiments which are important to be aware of and that can affect the spectral intensities, namely self-absorption, which we denote as saturation effects, giving rise to a non-linear intensity scale for XAS and non-dipole contributions in XRS, which mainly affect the pre-edge intensity. We will below describe the different detection schemes for XAS and also how well XRS and XAS can be compared in terms of the spectral line shape. We will also discuss the problems with each detection scheme and how to correct or avoid a non-linear intensity scale or at least keep the deviation of the non-linear effect constant as other parameters are changed in the experiment and thereby obtain a correct relative intensity scale.

\section{II.1 Detection methods of x-ray absorption spectroscopy}
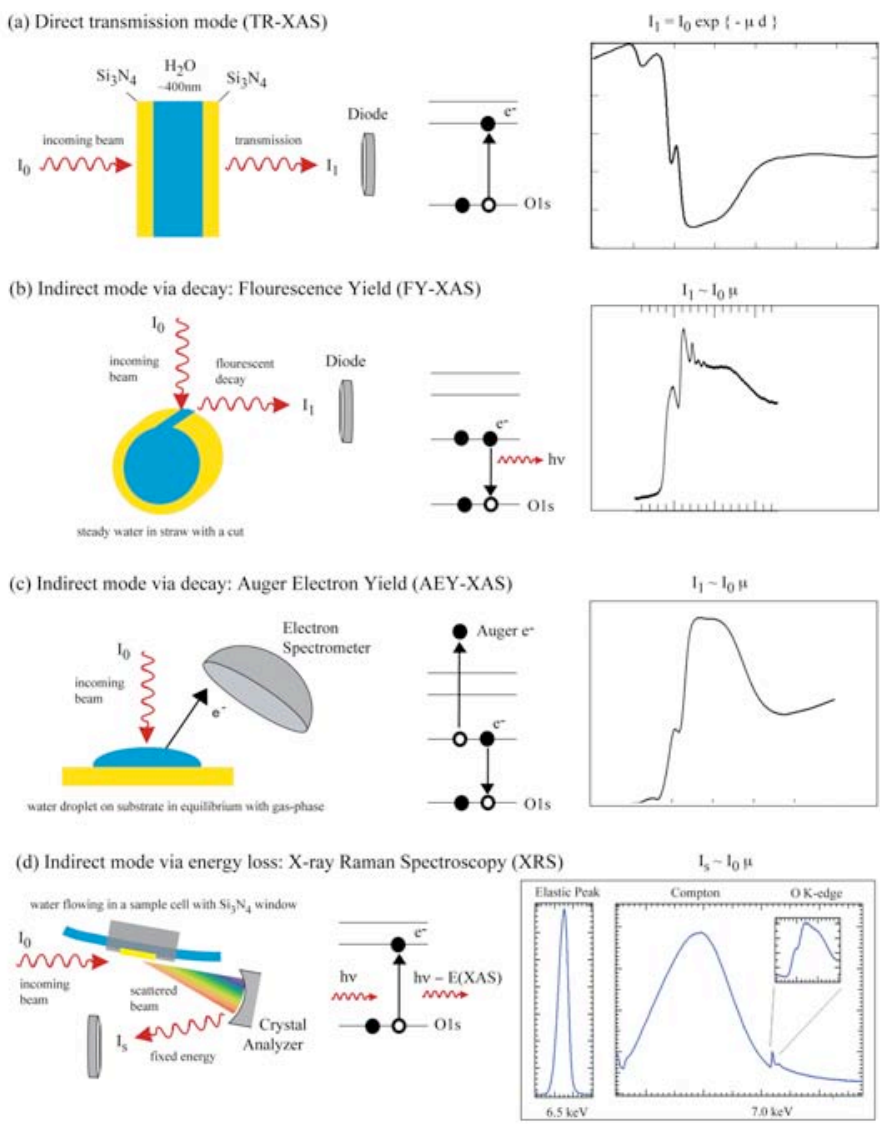

Fig. 3 The four different measurement methods of XAS and XRS of water shown in three columns: experimental setup, physical process and detected spectrum of liquid water for a) transmission, b) fluorescence yield, c) Auger yield and d) x-ray Raman as an inelastic process.

Fig. 3 shows the principles of measuring XAS using different detection schemes as either transmission or secondary yield techniques, using fluorescence or Auger detection, and of x-ray Raman scattering (XRS) (43). The most direct way to measure XAS is using transmission (fig. 3a) where the intensity is monitored after absorption in a thin cell and by using eq. (1) to determine the absorption coefficient $\mu(\omega)$ as the photon energy varies (43). 


$$
I_{1}(\omega)=I_{0} \exp (-\mu(\omega) x)
$$

The measurement is conducted by squeezing a small amount of water between two thin $\mathrm{Si}_{3} \mathrm{~N}_{4}$ membranes. The transmitted intensity $\left(\mathrm{I}_{1}\right)$ is measured using a diode behind the sample and the incoming intensity $\left(\mathrm{I}_{0}\right)$ needs to be determined using an empty cell. The obtained spectrum is then measured as transmitted intensity where increasing absorption causes a decrease in the transmission, see fig. $3 \mathrm{a}$. In order to determine the absorption coefficient $\mu(\omega)$ the logarithm of the transmission spectrum is taken after proper normalization has been obtained by dividing by $\mathrm{I}_{0}$. In order to obtain a spectrum where the most intense spectral features do not cause very low transmission and where the intensity of the continuum at higher energy can be determined with high accuracy it is important that the thickness of the water film is limited to between 300-500 $\mathrm{nm}$. The challenge is to produce such a thin sample and also to obtain a uniform thickness over the area sampled by the beam. In fig. 4 we show transmission spectra of water that have been obtained either using a beam with a reduced spot size as obtained with a pin-hole of $50 \mu \mathrm{m}$ or using a Scanning Transmission X-ray Microscope (STXM) with a focus of $50 \mathrm{~nm}$ as obtained from a zone-plate (44). As we can see there are deviations between the two transmission measurements which are due to the non-uniform thickness within the larger area sampled to obtain the normal transmission spectrum. In the STXM measurement the focus is much smaller than any length scale of the thickness variation which eliminates this uncertainty and provides the perfect conditions for transmission measurements. If several STXM spectra taken at different spots are summed together before the logarithm of the transmitted intensity is taken, a similar spectrum as from the normal transmission measurements is obtained as shown in fig. 4; the nonuniformity causes some saturation effects at the main-edge. Therefore, if perfectly flat and uniform samples cannot be generated, the most direct and reliable XAS transmission measurement of water is obtained when using the STXM set-up.

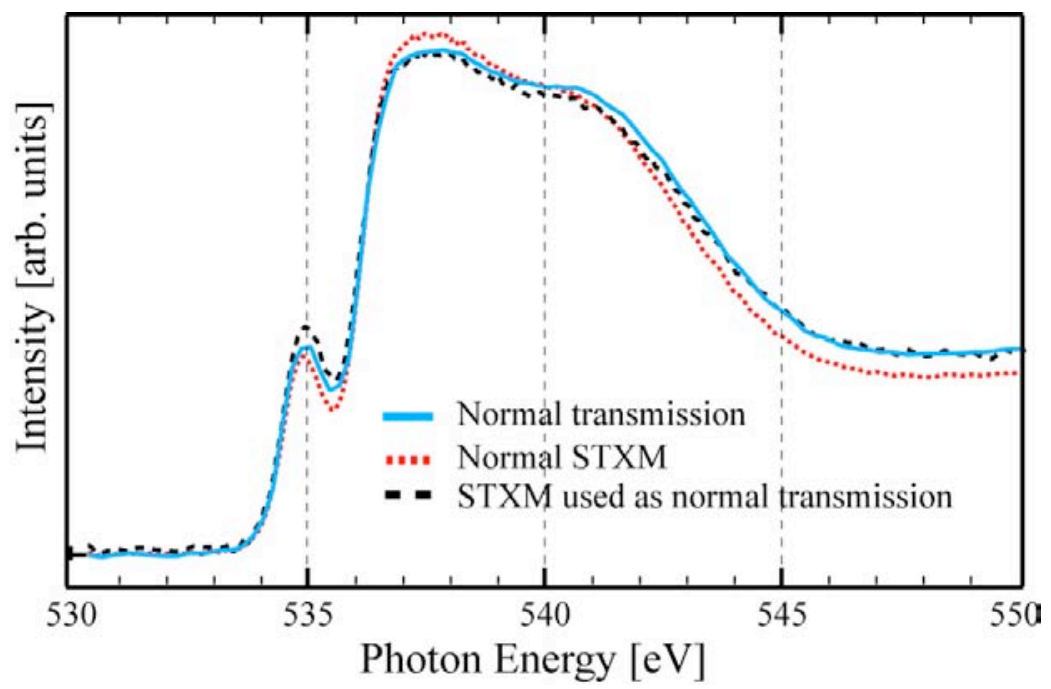

Fig. 4 Comparison of XAS transmission spectra of water measured with a $50 \mu \mathrm{m}$ pinhole (normal transmission, blue line), STXM (normal STXM, red line) and after summing several STXM transmission curves at different locations of the sample with different thicknesses before the logarithm is taken to obtain the XAS spectrum (STXM used as normal transmission, black line). 
The other detection modes of the XAS spectrum use an indirect method via the secondary process where the generated core hole decays via either radiative or non-radiative processes (40). The radiative detection scheme is often denoted fluorescence yield (FY) (fig. 3b) while the nonradiative mode (fig. 3c) can be divided into Auger-electron yield (AEY) and total electron yield (TEY) detection. Here we assume that the absorption within the detected layer is rather low allowing for a Taylor expansion of $\exp (-\mu(\omega) \mathrm{x})$ where the higher order terms can be neglected. This means that the detected signal in terms of core hole decay events is directly proportional to the absorption coefficient $\mu(\omega)$. If, on the other hand, the layer from which the secondary process is detected is rather thick, going beyond the linear regime, we could have saturation effects where the spectral intensities of the most intense features are suppressed (45-47). In the nonradiative process the core hole decays via the Auger process, giving rise to a high-kinetic energy Auger-electron which is detected via an energy dispersive electron analyzer to yield the AEY signal. Usually the penetration depths of soft x-rays are much longer than that for electrons. This would mean that the detected layer is much thicker in the case of FY than when using electron yield. Although the first high-resolution XAS of water was conducted with FY by detecting the emitted fluorescent photons at extremely grazing angle in order to decrease saturation effects by minimizing the thickness of the detected layer, the final spectrum still had severe saturation effects (18). It is, however, possible to correct for saturation effects if the attenuation of the emitted fluorescent light can be estimated $(48 ; 49)$. It was shown in Näslund et al. that the FY spectrum of water after correction for saturation effects becomes rather similar to the transmission XAS and XRS spectra (48).

For solid materials the most commonly used detection techniques are AEY and TEY since the surface sensitivity provides a thin detection region and thereby saturation effects can be avoided. It might therefore seem to be a most attractive alternative to the complex transmission set-up with ultrathin samples and to avoid the problem with corrections of saturation effects with FY. Typically for solid samples AEY gives the highest surface sensitivity in comparison to TEY since within the material the majority of the Auger-electrons are scattered, which gives rise to a scattering cascade of low-kinetic energy electrons with a longer mean free path. Using AEY detection the Auger electrons which have not undergone significant energy loss are detected using an energy dispersive electron spectrometer as shown in fig. 3c (50). It has been observed that the AEY water XAS spectrum is different in comparison to the other detection modes, showing more intensity in the post-edge region (43). It is not clear at this moment if this should be attributed to the high surface sensitivity and that the structure in the surface region is different from the bulk. In the case of TEY all electrons are detected and the surface sensitivity can vary to a large degree depending on the kinetic energy of the electrons. It has been determined in theoretical simulations of the Auger cascade process in water that each Auger electron gives rise to 20 secondary electrons with many inelastic scattering events (51). The detected electron signal can thereby have a very large contribution of low energy electrons. Fig. 5 shows a comparison of water XAS spectra measured in transmission using STXM, which do not have any saturation effects, and an electron yield spectrum measured in 1 atm of He gas. The lowest excitation energy in $\mathrm{He}$ is $21.2 \mathrm{eV}$ giving a long mean free path for electrons with energy below this value, while at higher kinetic energy the electrons are strongly scattered. The gas thus acts as a filter and the resulting spectrum has extremely large saturation effects where the whole main- and post-edge regions are suppressed indicating a depth of detection comparable to the depth of absorption. The long escape depth of the electrons is due to the existence of a band gap in water and electrons with a kinetic energy below that will, similar to the case of the He gas, have a very 
low scattering cross section. In the TEY detection we can therefore have saturation effects if there is a large fraction of low-energy electrons in the detection scheme. It was argued that this was the cause for the different temperature dependence in Smith et al. in comparison with XRS measurements (19). In order to change the temperature in the TEY liquid jet set-up, the measurement position with respect to the nozzle was varied. This can lead to variations in jet size, in local geometry with respect to the incident light and detected electrons and in local gas phase pressure. In particular the spectra of Smith et al. showed a small increase in the pre-edge and a large decrease in both the main- and post-edge with increasing temperature contrary to the temperature dependence in the XRS spectra and to the difference between the spectra of ice and water $(19 ; 24)$. The observed large decrease in the main- and post-edge spectral features follows exactly the expected dependence for a variation of saturation effects in the spectra (19). For TEY measurements of thin layers of ice on surfaces saturation effects can often be avoided by ensuring that the film thickness is small (below $100 \AA$ ), i.e. smaller than the penetration depth of the incident photons.

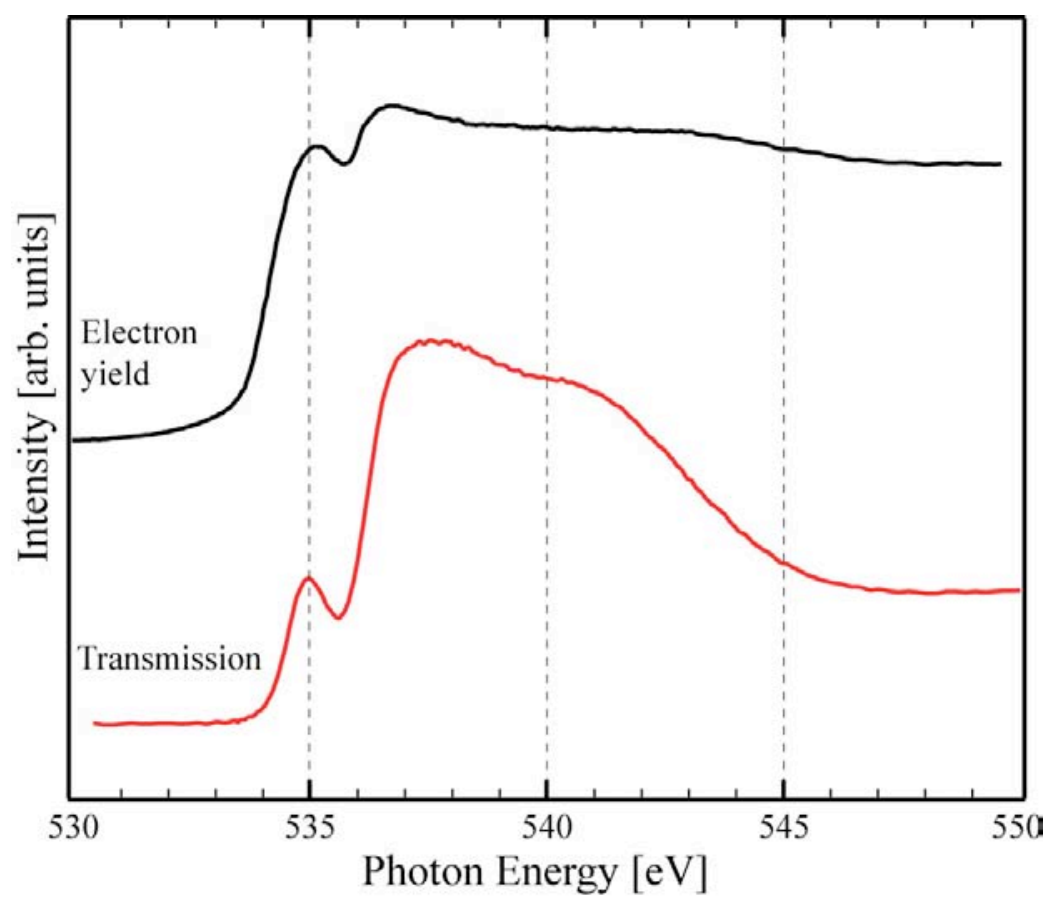

Fig. 5 Comparison of XAS transmission spectra of water measured with STXM (red) with XAS measured with total electron yield detection mode in $1 \mathrm{~atm}$ of He gas using a Au wire as electron detector with a bias of $+5 \mathrm{~V}$.

Energy calibration can usually be conducted using absorption edges from compounds that are well known or by using special techniques to measure differences in kinetic energies of photoemitted electrons excited by first and second order light coming out from the diffraction grating in the monochromator (52). The difference is the absolute photon energy. This requires an electron analyzer and has typically been done for the ice samples to obtain the energy scale (30). In the case of the energy calibration of the water spectrum in Myneni et al. the measurements were conducted in such a way that the gas phase spectrum was obtained on top of the liquid spectrum $(18 ; 48)$. The energy scale is then obtained relative to the gas phase. 


\section{II.2 Principle of X-ray Raman scattering}

The XRS technique has many advantages over the different direct XAS measurements due to the simple sample environments when using hard x-rays and the high reliability in the relative intensities of the spectral features. In an inelastic x-ray scattering experiment a small fraction of the incident photon energy is transferred to the sample. Besides its application to small energy transfers, as in vibrational (10-500 meV) and valence band excitations (1-10 eV), inelastic scattering of hard x-ray photons permits electronic excitations of core levels in the sample $(\sim 50-1000 \mathrm{eV})$. This scattering, usually referred to as XRS, allows the study of absorption spectra with edge energies lying in the soft x-ray regime by using hard x-rays. Thus the XRS technique has advantages over the different direct XAS measurements due to the much longer penetration depth of hard X-rays $(0.25-0.5 \mathrm{~mm}$ in water), which allows for more flexibility and less stringent requirements on both the sample and its immediate environment. In addition, because the XRS process is non-resonant, the scattering cross section varies very little across the near-edge region. This results in a very high reliability in the intensity scale and no saturation effects.

The close connection between XRS and XAS was established in the theoretical work by Mizuno and Ohmura (53) and experiments by Suzuki (54) in the late 1960s. In particular, the transition probability for XRS can be related to the same matrix element that determines x-ray absorption as long as the magnitude of the momentum transfer $\boldsymbol{q}$ of the inelastically scattered photon is small (vide infra). The main difficulty of XRS is the extremely small scattering cross section, and it was not until the emergence of powerful synchrotron sources, and the development of efficient spectrometers much later, that the advantages of XRS started to impact core hole spectroscopy beyond demonstration experiments (for two reviews on some of the earlier work see e.g. $(55 ; 56))$. In the last few years there has been a very large surge in the number of XRS studies (see e.g. the many references in (57)) including numerous studies on water, aqueous systems, and ice under ambient and extreme conditions $(3 ; 26 ; 32 ; 42 ; 43 ; 48 ; 57$ $64)$.

In XRS the scattered $\mathrm{x}$-rays are detected with a high-resolution perfect-crystal spectrometer (see schematics in fig 3d), and most of the current devices are based on Bragg reflections from multiple spherically curved analyzers in a backscattering Rowland geometry. Such instruments combine good energy resolution $(0.1-1 \mathrm{eV})$ with a large acceptance angle, yet the extremely low XRS cross section often requires a compromise in terms of resolution versus signal intensity. Because the energy resolution of XRS is determined by the convolution of both incident and scattered x-rays, many of the XRS experiments on water were performed with high flux Si (111) monochromators, hence an energy resolution limited to $\sim 1 \mathrm{eV}$. Some higher resolution studies have been reported $(26 ; 60)$ and recently we were able to obtain very high quality spectra of the temperature dependence in water with $\sim 0.5 \mathrm{eV}$ resolution using a 14crystal spectrometer that captures $0.9 \%$ of the $4 \pi$ sr solid angle (38). This allowed for the observation of temperature-dependent changes in the position and line shape of the pre-edge and main-edge spectral features (38) as will be discussed in section V.1.

Besides being complementary to XAS in terms of probing depth and sample environment, XRS has an additional spectroscopic property, namely the ability to probe the density of unoccupied states beyond the dipole limit that governs XAS. For a powder or amorphous system the double-differential cross section describing XRS is proportional to the dynamic structure factor 
$\mathrm{S}(q, \omega)=\sum\left\langle\left.\left\langle f\left|\mathrm{e}^{i q \boldsymbol{r}}\right| i\right\rangle\right|^{2} \delta\left(E_{f}-E_{i}+\hbar \omega\right)\right.$,

where $i$ and $f$ refer to the initial and final states, the sum is over the final states, $\hbar \omega$ is the energy loss, and $E_{i}, E_{f}$ are the incident and scattered photon energies, respectively. As compared to XAS, the x-ray polarization vector is replaced in XRS by the momentum transfer vector $\boldsymbol{q}=\boldsymbol{k}_{\boldsymbol{i}}$ $\boldsymbol{k}_{\boldsymbol{f}}$. Here $\boldsymbol{k}_{\boldsymbol{i}}$ and $\boldsymbol{k}_{\boldsymbol{f}}$ are the wave vectors of the incident and scattered x-rays respectively and the magnitude of $\boldsymbol{q}$ is given by $q=\left(k_{i}+k_{f}\right) \sin (\theta / 2)$, where $\theta$ is the scattering angle. The fact that $q$ can be large requires the expansion of the exponential in the matrix element beyond the dipole contributions when the condition $\boldsymbol{q r}<<1$ is not valid. These effects have been first studied on $\mathrm{Li}$ by Krisch et al. (65), and numerous recent studies have focused on investigating non-dipole contributions both experimentally and theoretically, including the work on water and ice by Fister et al. (57). For $1 s$ electrons the approximate magnitude of $\boldsymbol{r}$ is given by the spatial extension of the core hole wave function $r \sim a / Z$, where $\mathrm{a}=0.53 \AA$ is the Bohr radius and $Z$ the charge of the absorbing atom. In the first XRS study of water with sufficient resolution to resolve the pre-edge (32), the spectra were taken integrated over a range of $0.36<q r<0.57$, which was later found not to be in the strict dipole limit (3). The non-dipole contributions in the O K-edge of water mainly result in an enhancement of the pre-edge due to $s \rightarrow s$ transitions as will be further discussed in section III.5.

Taking XRS spectra at large $q$ might help to enhance small spectral changes (in particular in the pre-edge region) and there is an important practical advantage: the strength of the XRS signal is approximately proportional to $q^{2}$. This can be varied by either changing $\theta$ or the magnitude of $\boldsymbol{k}_{\boldsymbol{i}}$ and $\boldsymbol{k}_{\boldsymbol{f}}$ by way of the incident x-ray energy $E_{i}$. Based on such simple considerations going to higher $E_{i}$ might seem to be the obvious choice; it even further enhances the XRS signal by way of a larger scattering volume. However, there is a severe experimental limitation because current perfect-crystal XRS spectrometers become both less efficient and poorer in energy resolution at higher energies. In addition, the Compton background becomes larger, changing the signal to noise unfavorably. Another practical consideration for the best choice for $\theta$ is given by the fact that synchrotron radiation is linearly polarized in the horizontal direction and there is a $\cos ^{2} \theta$ polarization dependence of the scattered intensity in a horizontal XRS scattering geometry. Therefore, ideally, XRS scattering is performed in a vertical scattering plane where the polarization factor becomes one. However, due to practical reasons related to the horizontal beam size, energy resolution and sample alignment, XRS experiments are sometimes carried out in a horizontal scattering geometry.

In order to determine the choice of the optimal incident energy as well as scattering angle several parameters have to be considered, in particular a) whether or not the XRS is to be directly compared to XAS, b) what is the scattering geometry, c) what is the required energy resolution, d) what is the required penetration through the sample environment, e) how large is the sample volume, and f) what is the concentration and type of the solvent (in case of aqueous solutions). To date all reported XRS experiments on water and aqueous solutions have been carried out at incident energies in the $7-10 \mathrm{keV}$ range and with a resolution between $0.5-2 \mathrm{eV}$.

\section{II.3 Comparing x-ray absorption and x-ray Raman measurement methods}

In order to summarize the discussion of the experimental aspects of XAS/XRS we conclude that the most reliable methods for measuring XAS are either by transmission mode on a homogeneous sample or by using STXM to ensure measured regions of the sample with a 


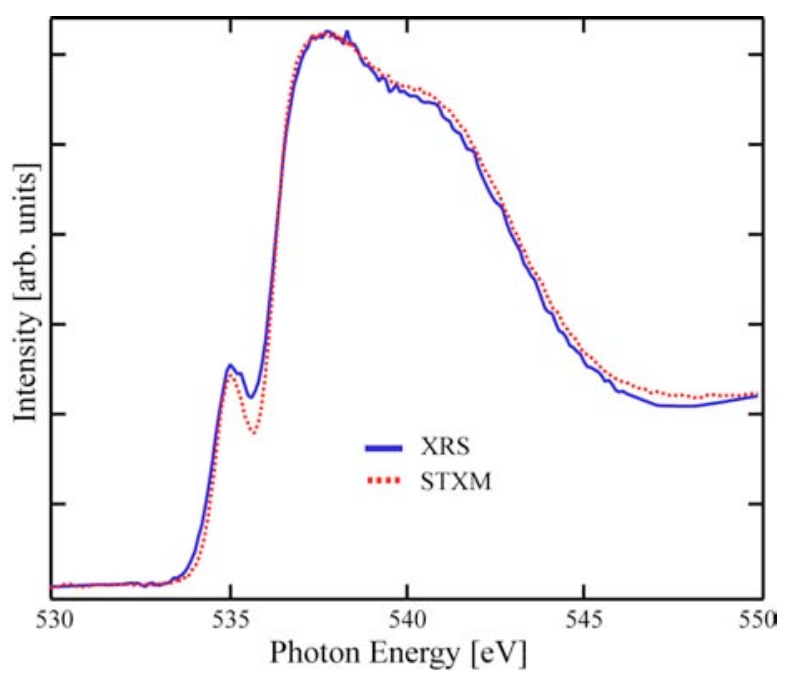

Fig. 6 Comparison of XAS transmission spectra of water measured with STXM (red) with an energy resolution of $0.1 \mathrm{eV}$ and XRS (blue) with a total resolution of $0.5 \mathrm{eV}$.

perfectly uniform thickness of around 300-400nm or XRS at low $q$-transfer. Fig. 6 compares high-resolution XRS and transmission XAS with STXM spectra showing in general good agreement. We can note the better resolved pre-edge feature in the XAS spectrum which is attributed to the higher resolution of around $0.1 \mathrm{eV}$. However, we also observe a somewhat higher intensity of the pre-edge feature in the XRS spectrum, which could be due to a small nondipole contribution even at the low momentum transfer condition. In the case of studies involving other detection schemes with the objective to follow relative spectral changes induced by temperature, pressure and various concentrations of solutes it is extremely essential that the experimental spectra are fully reproducible. Fig. 7 shows transmission spectra of pure water measured in 3 different runs separated by several months and where the experimental chamber has been moved in and out at the beam line at each occasion. As the figure shows, the data are fully reproducible although some weak saturation effect is present due to the non-uniform thickness of the water film. This level of reproducibility was only achieved after inserting a pinhole with diameter ranging from $10-50 \mu \mathrm{m}$ in front of the sample in order to measure regions of the sample where the thickness is uniform.

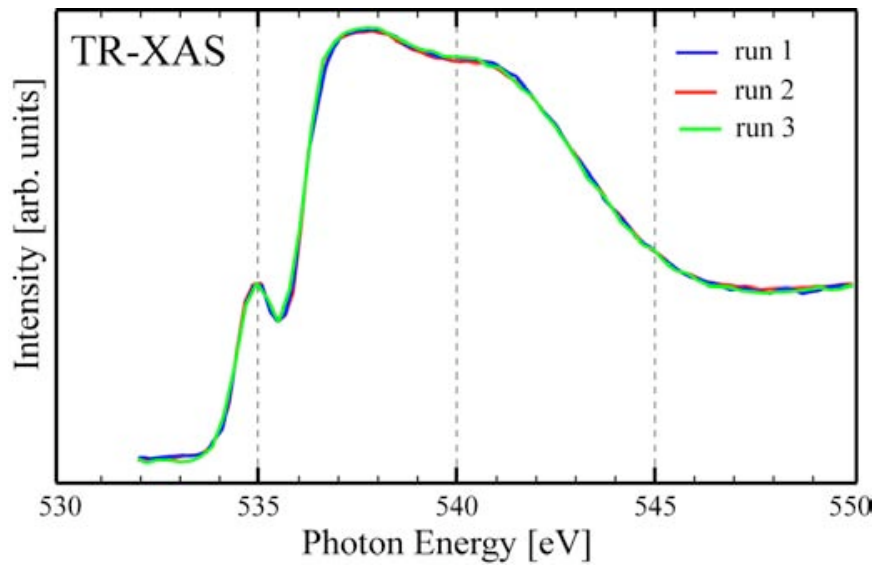

Fig. 7 XAS spectra of water measured in transmission mode from three separate beamtimes, showing reproducibility (red: December 2008, $50 \mu \mathrm{m}$ pinhole; blue: May 2009, $10 \mu \mathrm{m}$ pinhole; green: July 2009, $25 \mu \mathrm{m}$ pinhole). All spectra were normalized by area from $532-550 \mathrm{eV}$. 


\section{Fundamentals of the Spectroscopy of Importance to Water}

Let us first go through some basic principles regarding $\mathrm{x}$-ray spectroscopy in general with an emphasis on aspects that are important for applications to water. The different concepts will be important for our understanding of the XAS/XRS spectra of water, adsorbed layers and various ices that will be discussed in sections IV and V.

\section{1 Basic principles of $x$-ray absorption spectroscopy}

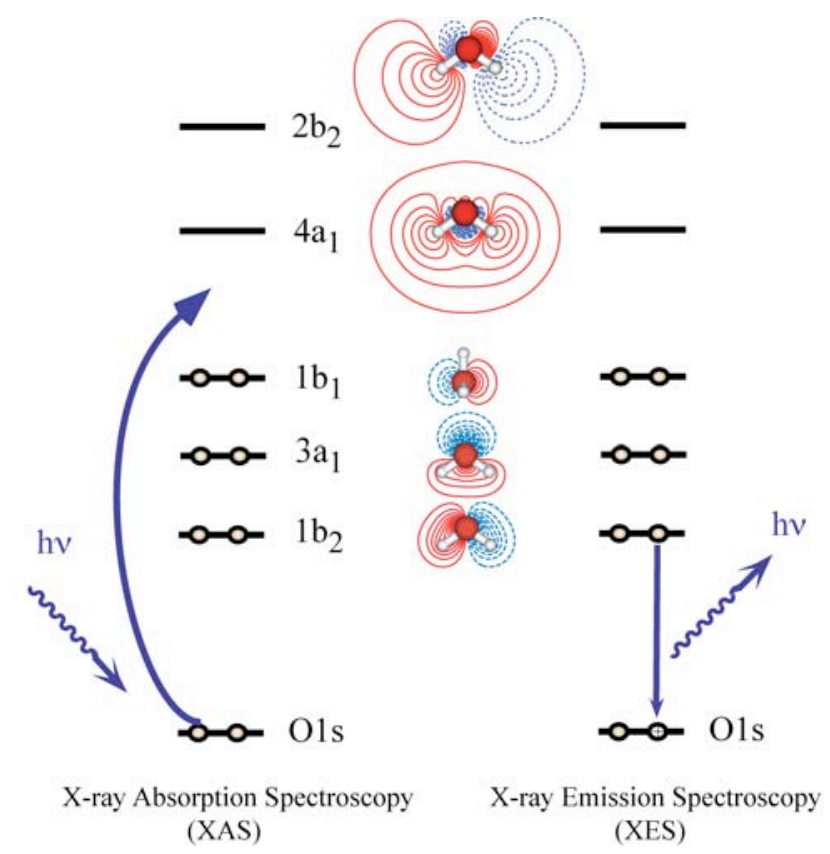

Fig. 8 Schematic orbital diagram of a free water molecule illustrating the principles for $\mathrm{x}$-ray absorption spectroscopy and $\mathrm{x}$-ray emission spectroscopy.

Fig. 8 shows a molecular orbital diagram of the free water molecule relevant for our discussion of XAS/XRS and XES. The three outermost occupied orbitals are the bonding $1 b_{2}$ and $3 a_{1}$ and the non-bonding $1 b_{1}$ lone-pair. To the two bonding orbitals corresponds a set of orthogonal orbitals of antibonding character which are denoted $4 a_{1}$ and $2 b_{2}$ and which are unoccupied in the ground state. Fig. 8 illustrates the two x-ray spectroscopies through a process where an electron is either excited from the $\mathrm{O} 1 s$ core orbital to the unoccupied orbitals or an electron decays into the $\mathrm{O} 1 s$ core hole from the occupied orbitals. The former is x-ray absorption where a photon is absorbed. The same excitation can occur through x-ray Raman scattering in which instead a high energy photon undergoes an energy loss where the energy is given to the excitation process from the $\mathrm{O} 1 s$ to the unoccupied states. The decay of the excited state illustrated in fig. 8 is the x-ray emission process where a photon is emitted with energy corresponding to that released from the decay process. One thing that we immediately note in fig. 8 is the large difference in size between the occupied and unoccupied orbitals. The occupied orbitals are more confined in close proximity to the molecule whereas the unoccupied orbitals have larger spatial extent, in particular around the hydrogen atoms. This results in a large difference in sensitivity to H-bonding between the two spectroscopies. As will be discussed in section III.4 the XAS spectrum changes dramatically due to H-bonding when other molecules come close and overlap between the unoccupied orbitals causes rehybridizations and energy 
shifts. On the other hand the occupied orbitals will be much less sensitive to H-bonding since the overlap with other molecules is much smaller. This is also observed in the x-ray emission spectra (XES) of water $(14 ; 25)$ and also in the corresponding photoelectron spectra where the valence electrons are directly ionized $(66 ; 67)$. In the XES spectra there is an interesting split in the $1 b_{1}$ orbital spectra which is mainly due to a core level shift of the $\mathrm{O} 1 s$ which will affect the energy of the decay (25).

The primary focus of this article is on the XAS and XRS at the dipole limit but many aspects are the same for XES. The most essential aspect of the spectroscopic process is that it provides an atom-specific probing of the electronic structure around a specific site coupled with the dipole selection rule. This comes from the unique property to probe the valence electrons close to the core electron shell where the matrix element in the transition becomes atomic-like. It forms the basis for the "one-center approximation". Let us illustrate in a simple manner this principle for the carbon monoxide $(\mathrm{CO})$ molecule. First we need to consider the transition matrix element for x-ray absorption.

$$
I \propto\left|\left\langle F\left|\vec{D} \bullet \overrightarrow{E^{\prime}}\right| I\right\rangle\right|^{2}
$$

The initial and final state wave functions contain all electrons in the whole system. We may attempt to approximate the matrix element over the determinantal wave functions $|F\rangle$ and $\mid I>$, representing the final and initial states, in such a way that we separate the one-electron core $\phi_{c}$ and the excited orbital, $\phi_{v}$, that are directly involved in the excitation process from the rest of the system, $\Psi_{\mathrm{f}}$ and $\Psi_{\mathrm{m}}$. We distinguish the one-electron part from the many-body part of the matrix element in the following way:

$$
I \propto\left|\left\langle\phi_{v}\left|\vec{D} \cdot \vec{E}^{\prime}\right| \phi_{c}\right\rangle\left\langle\psi_{f} \mid \psi_{m}\right\rangle\right|^{2}
$$

The term $\left\langle\Psi_{\mathrm{f}}\right| \Psi_{\mathrm{m}}>$ is the codeterminant of orbital overlaps obtained by eliminating the orbitals $\phi_{v}$ and $\phi_{\mathrm{c}}$ from the full determinant. In the case that the remaining orbitals are only slightly perturbed by the transition then the codeterminant will be approximately unity and the cross section can be approximated by the single term shown in eq. 4 . If we assume that this is the case we can furthermore neglect the many-body overlap term and the matrix element is reduced to an overlap between the core and valence orbitals weighted with the dipole operator.

Fig. 9 shows the $1 s$ and $2 p$ wave functions for the $\mathrm{C}$ and $\mathrm{O}$ atoms in a $\mathrm{CO}$ molecule (34), which are the orbitals involved in the determination of the transition moments in eq. 4 . The atoms are positioned relative to each other at $1.15 \AA$ corresponding to the bond distance in CO. We observe the strong overlap of the $\mathrm{O} 2 p$ and $\mathrm{C} 2 p$ wave functions which will lead to the formation of molecular orbitals. The energy positions of the resulting molecular orbitals will depend on the nature of this overlap. If we inspect where in the atoms the $1 s$ levels overlap with the valence orbitals we see that it takes place deep inside the atom in the region of the core electrons. This region will make the largest contribution to the matrix element in eq 4 . There are no significant interatomic contributions between the $\mathrm{O} 1 s$ and $\mathrm{C} 2 p$ or $\mathrm{C} 1 s$ and $\mathrm{O} 2 p$ orbitals. The contribution to the one-electron matrix element comes only from the particular center on which the core electron is located; the magnitude of off-center $2 p$ orbital contributions is determined 


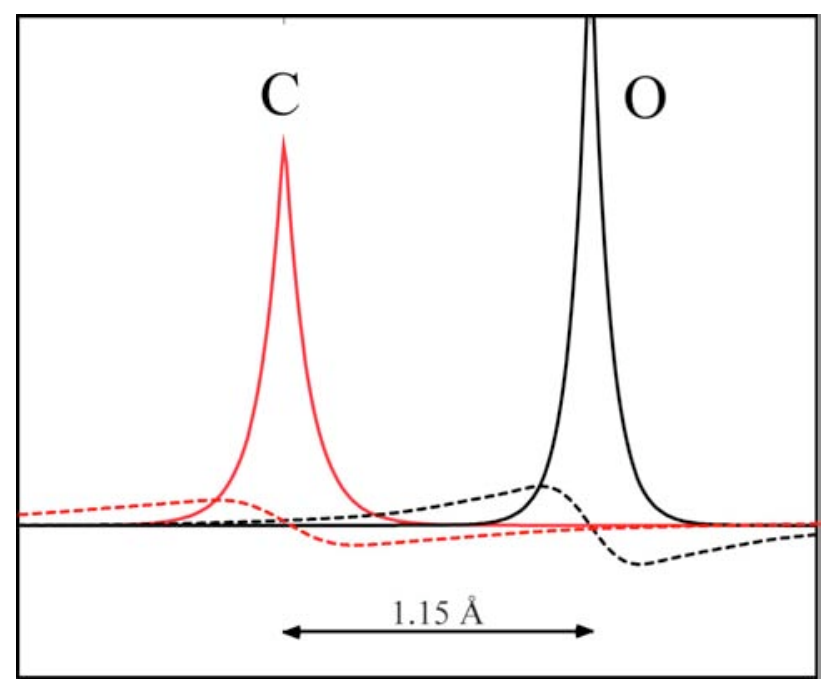

Fig. 9 Orbital amplitudes along the bond axis of the atomic (dashed lines) $\mathrm{C} 2 p, \mathrm{O} 2 p$, and (solid lines) $\mathrm{C} 1 s$ and $\mathrm{O}$ $1 s$ wave functions with $\mathrm{C}$ and $\mathrm{O}$ atoms at the interatomic distance, $1.15 \AA$, of the CO molecule (34). Note that the amplitudes are normalized to give the correct atomic orbital normalization over volume.

through the amplitude over the core region and this is extremely small. We note then that the transition moment is related to the atomic part of the molecular orbitals whereas the energy of the transition will be more connected to the extended part of the molecular orbital where overlap between different atomic centers takes place. The intensity of the x-ray absorption transition is thus directly related to the partial population of atomic $2 p$ character in the different unoccupied molecular orbitals and we can then apply a simple atomic picture of the dipole selection rule where the change in angular momentum in the x-ray transition is given by:

$$
\Delta l= \pm 1
$$

From $s$ core electron absorption we probe unoccupied local $p$ character in the molecular orbitals; that is, we measure the projection of the atomic $p$ orbital contribution to the molecular orbitals through the intensity of the transition as seen in the spectrum.

\section{2 The water gas phase spectrum}

Let us now take a look at the gas phase XAS spectrum of the water molecule, $\mathrm{H}_{2} \mathrm{O}$, as shown in fig. 10 (18). The two lowest transitions correspond to excitations into the $4 a_{1}$ and $2 b_{2}$ molecular orbitals (40). The intensity of the transitions reflects the amount of $\mathrm{O} 2 p$ character in these orbitals. Since all the occupied orbitals in the water molecule are strongly polarized towards the oxygen atom due to the difference in electronegativity, then, due to orthogonality, the antibonding unoccupied orbitals $4 a_{1}$ and $2 b_{2}$ are dominated by contributions from the hydrogen atoms. An inspection of fig. 8 shows that the orbitals extend far outside the water molecule on the hydrogen atom sides. This comes also from the antibonding character which pushes the charge distribution in the orbitals away from the $\mathrm{OH}$ bonds. The intensities of the excitations into the first molecular orbitals in the water molecule are much lower in comparison to transitions into molecular orbitals of a homonuclear molecule such as the $\pi$ excitation in $\mathrm{O}_{2}$ (40) due to the greater $\mathrm{O} 2 p$ orbital population in water leaving less unoccupied $2 p$ character. 


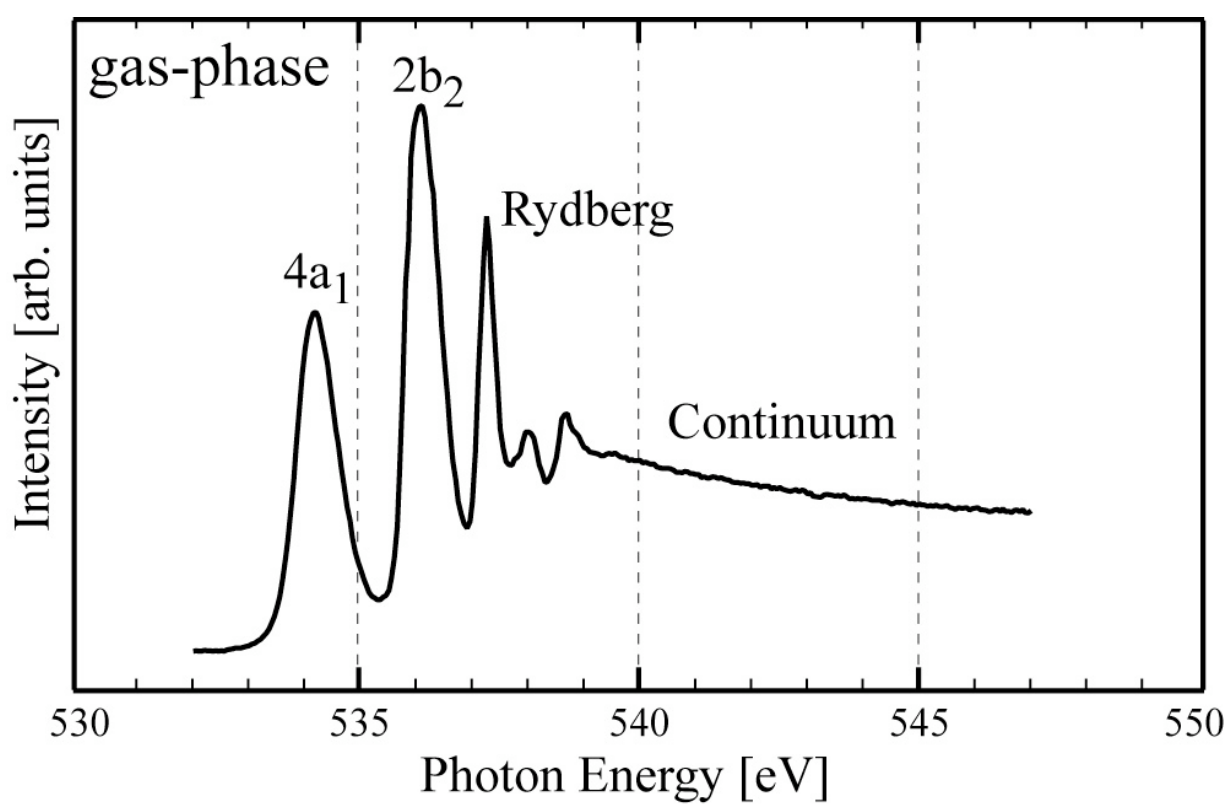

Fig. 10 XAS spectrum of water in the gas phase measured with fluorescence detection (18).

At higher energies we see a series of very sharp peaks which come from excitations into Rydberg orbitals. These are mainly composed of $\mathrm{n} p$ orbitals from higher shells such as $3 p, 4 p$ etc. Since the wave functions of the higher shells are located further out from the core they will give rise to smaller overlap with the $1 s$ orbital and thereby give lower intensities. These Rydberg excitations converge towards the water molecule ionization potential at $539.6 \mathrm{eV}$ (68). At energies above the ionization energy only a straight line is observed in the spectrum which corresponds to excitations into the continuum with an outgoing free electron wave. The intensity decreases to higher energies since the wave length of the outgoing wave decreases causing smaller overlap with the core. In some molecular systems such as $\mathrm{N}_{2}, \mathrm{CO}$ and $\mathrm{O}_{2}$ the antibonding $\sigma$ orbitals are pushed up in energy and appear above the ionization threshold (40). This is no longer a bound state and is instead viewed as a resonance where the excited electron rattles around within the centrifugal barrier of the molecular potential before it escapes as a free electron above the vacuum level (40). These states are therefore often denoted shape resonances. The free water molecule has no such resonances but upon H-bonding there are states pushed up in energy and these have properties similar to the shape resonance seen for orbitals in small molecules. This will be discussed in section III.7.

\section{3 Vibrational excitations}

The time scale of the excitation process is very short compared to nuclear motions. The interaction between two states separated by an energy $\Delta \mathrm{E}$ evolves over a characteristic time $\mathrm{t}$ of an optical cycle $\mathrm{t} \Delta \mathrm{E} \sim \mathrm{h}$, where $\mathrm{h}=4.136 \mathrm{fs} \mathrm{eV}$. For an $\mathrm{O} 1 s$ excitation process with $\Delta \mathrm{E} \sim 530 \mathrm{eV}$, the excitation time scale becomes $\mathrm{t} \sim 8$ as (20). This is much shorter than any nuclear motions and we can therefore regard the nuclear positions as frozen during the excitation process. This is the basis of the Franck-Condon principle which is illustrated in fig. 11. This often leads to an $\mathrm{x}-$ ray spectroscopic process being accompanied by excitation of vibrations: If after core-excitation equilibrium interatomic distances and/or the force constants change, vibrational motion is excited in the final state. The ionization is usually treated within the Born-Oppenheimer approximation, 
which implies that the nuclear motion can be decoupled from the electronic motion. This allows the total wave function to be factorized into one nuclear and one electronic part, which are solutions to separate equations.

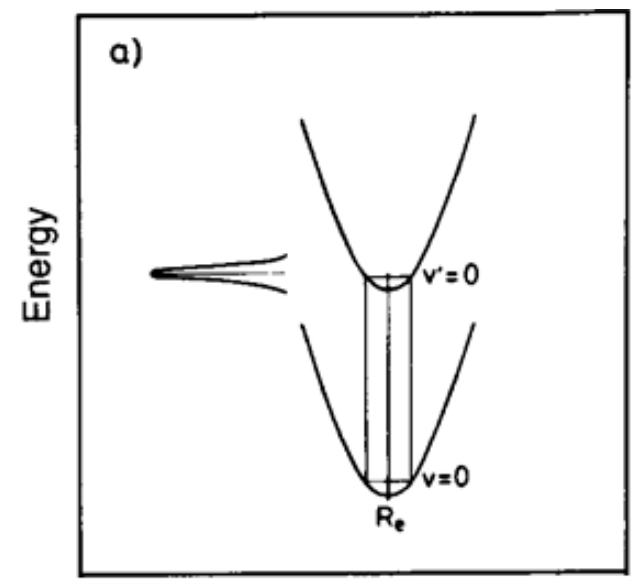

Bond distance

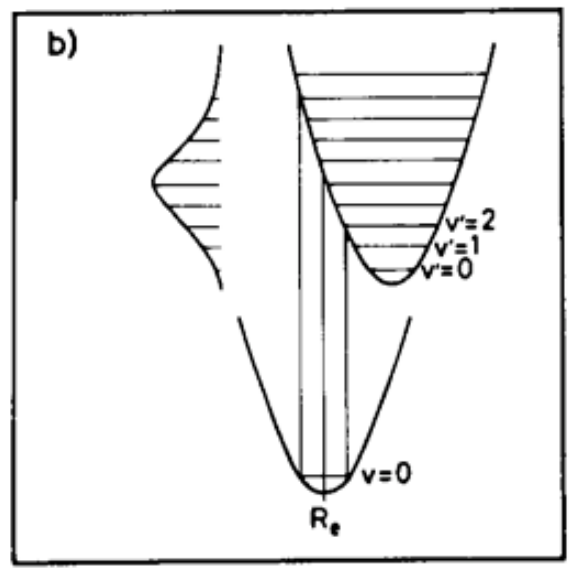

Bond distance

Fig. 11 Initial and final state potential energy curves describing x-ray absorption in two different cases: a) corresponds to a small and b) to a large geometry change due to the excitation process, giving rise to small and large line widths, respectively.

Fig. 11 shows the potential energy curves for the nuclear motion for two different cases. The lower curve in each figure corresponds to the initial state and the upper curve to the final state of a particular electronic transition, such as a core-ionization process. The vibrational states are characterized by quantum numbers $v$ and $v^{\prime}$ for the initial and final states, respectively. In fig. 11 it is assumed that only the $\mathrm{v}=0$ vibrational state is populated in the initial state. For the transition probabilities to the various vibrational states the Franck-Condon principle is generally applicable and states that the relative transition probabilities for the same electronic transition are given by the square of the overlap between the initial and final state nuclear wave functions. The situation in fig. 11a refers to a situation where the initial and final state potential energy curves are very similar. In this case only the $\mathrm{v}=0$ to $\mathrm{v}^{\prime}=0$ overlap is non-zero and the peak is very sharp as is the case for the Rydberg state at $537.2 \mathrm{eV}$ excitation energy in gas phase water. In fig. $11 \mathrm{~b}$ the potential energy curves are quite different and the $\mathrm{v}=0$ wave function is non-orthogonal to a whole series of final state wave functions with $v^{\prime}=0,1,2$ etc. This leads to the excitation of a number of different vibrational final states leading to broad spectral lines as seen in the $4 \mathrm{a}_{1}$ and $2 b_{2}$ peaks. Excitation into antibonding orbitals leads to much larger deviations of the final state equilibrium geometries which is seen as greater broadening of the spectral features in comparison to excitations into non-bonding orbitals such as Rydberg states. Furthermore, excitation into the $4 \mathrm{a}_{1}$ antibonding state leads to a final state potential energy curve which is dissociative where the hydrogen atom will be repelled with a kinetic energy depending on the initial nuclear position at the time of ionization (69-71).

The $2 b_{2}$ state on the other hand shows vibrational fine structure in the XAS spectrum as shown in fig. 12 (72). There are two different vibrational progressions corresponding to excitations of the $\mathrm{OH}$ stretch and bending modes. The equilibrium geometry of the core excited $2 \mathrm{~b}_{2}$ state is where the $\mathrm{O}-\mathrm{H}$ bond is elongated by $0.25 \AA$ and the $\mathrm{H}-\mathrm{O}-\mathrm{H}$ angle has become $10^{\circ}$ 
smaller. Fig. 12 shows the different $2 b_{2}$ line shapes of the $\mathrm{H}_{2} \mathrm{O}$ and $\mathrm{D}_{2} \mathrm{O}$ molecules due to the smaller vibrational energy in the heavier $\mathrm{D}_{2} \mathrm{O}$ molecule.

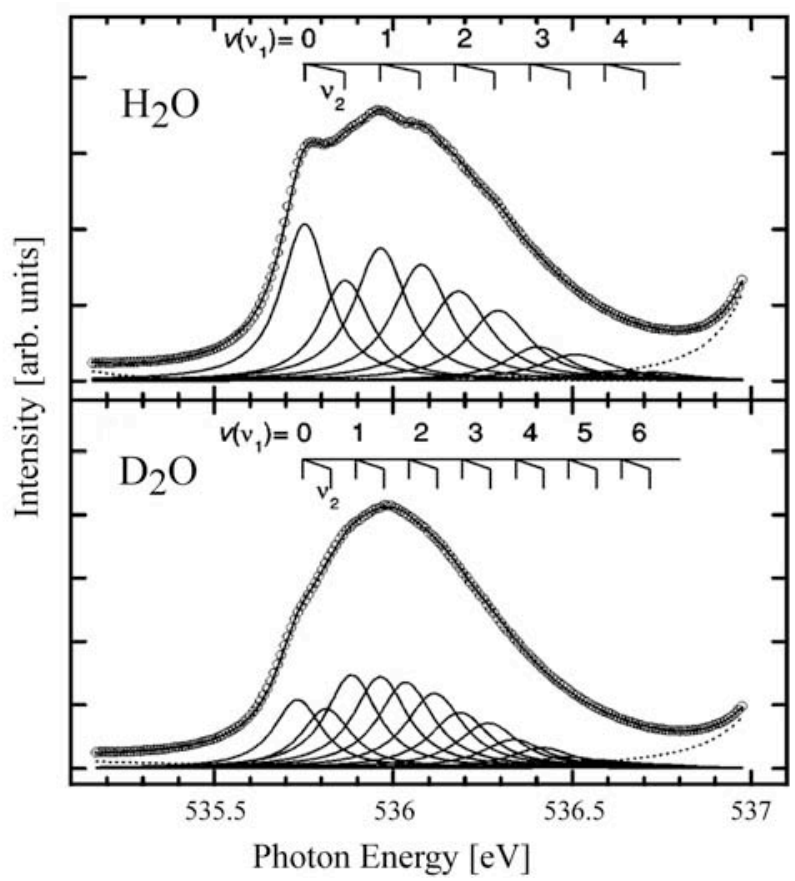

Fig. 12 High resolution gas phase spectra of the $\mathrm{H}_{2} \mathrm{O}$ and $\mathrm{D}_{2} \mathrm{O}$ molecular $2 \mathrm{~b}_{2}$ band (72). The solid curves are the results of a least-squares peak fit.

The importance of the Franck-Condon principle for XAS on liquid water is that all water molecules are frozen on the time-scale of the spectroscopic process. This means that the spectral information reflects a spatial average of frozen geometries of the molecules in the liquid with, in addition, an average in time corresponding to an average of snapshots of configurations at different times during the measurement. This is different in comparison to $\mathrm{OH}$ stretch vibrational spectroscopy where the time-scale of the excitation process is similar to the dynamical motion in the liquid complicating the interpretation (73). In spite of the ultrashort time-scale of the x-ray absorption process, vibrations still affect the line shape of the liquid water spectrum in the form of vibrational excitations of the internal bonds similar to the gas phase spectrum. We will discuss some of these in connection to the difference in spectral line shape between liquid $\mathrm{H}_{2} \mathrm{O}$ and $\mathrm{D}_{2} \mathrm{O}$ in section V.2.

\section{4 Spectral changes due to the formation of condensed phase}

We will next consider changes in the XAS spectra due to the formation of a condensed phase such as ice. Let us compare spectral changes upon condensation of water with those of a molecule such as methane $\left(\mathrm{CH}_{4}\right)$ which is similar to water in terms of its electronic structure. In the top part of fig. 13 the XAS spectrum of gas phase methane is shown (74). The spectrum shows a lot of resemblance with the gas phase water spectrum but with a richer, more resolved vibrational fine-structure. When we condense a thick layer of methane into ice on a $\operatorname{Pt}(111)$ surface at $30 \mathrm{~K}$ we observe rather small changes in the spectrum (75). There are some small additional broadenings, but most of the vibrational fine-structure is preserved. However, when we condense water into ice there are very large spectral changes as shown in the bottom part of 
fig. 13. The discrete spectral features of the $4 a_{1}$ and $2 b_{2}$ orbitals are nearly gone and instead a strong broad resonance, denoted the post-edge, develops at $5 \mathrm{eV}$ higher excitation energy than the $4 a_{1}$ state of the free molecule. The observed large spectral change upon water vapor condensation must be related to the formation of $\mathrm{H}$-bonds. The short intermolecular distance in $\mathrm{H}$-bonds must perturb the electronic structure of the water molecule leading to the formation of a completely different set of unoccupied molecular orbitals or, since we are dealing with a condensed phase, band structure.

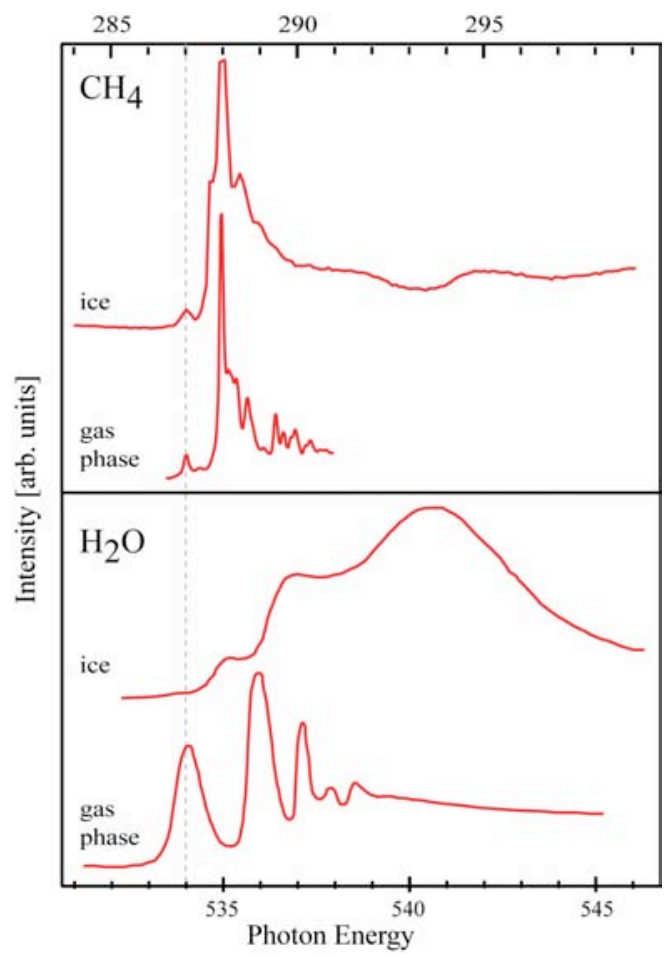

Fig. 13 XAS spectra of gas and ice phase water and methane $(30 ; 74)$. Note the two energy scales below and above the spectra for the $\mathrm{O} 1 s$ and $\mathrm{C} 1 s$ regions, respectively.

Although the vibrational fine-structure is still seen in the methane ice spectrum for the first few excited states no fine-structure is seen for the Rydberg orbitals around $289 \mathrm{eV}$ and above. Instead broad resonances appear in the spectra. The much larger radius of the Rydberg orbitals causes these to overlap with the surrounding molecules in the condensed phase lattice and thereby shifting their energy positions to form broadened resonances more resembling a band structure picture. In a simplified picture the larger C-C distance of $4.16 \AA$ (76) between the methane molecules in methane ice in comparison to the O-O distance of $2.75 \AA$ in water ice requires excited states involving orbitals with larger radius that can overlap with those of the surrounding molecules for spectral changes to occur. This is then exactly the case for Rydberg orbitals in methane whereas the same type of change is seen already for the more compact molecular orbitals in water. This is a clear manifestation of the difference in nearest-neighbor distance between methane and water ices in the respective XAS spectra. If we imagine that we could move water molecules to the same distance as in methane ice we would expect to observe intact and discrete excited states for the $4 a_{1}$ and $2 b_{2}$ orbitals in water ice as seen in the spectrum of methane ice. We can from this already conclude that for transformation back to the more discrete molecular orbitals seen as the pre-edge and main-edge features in the liquid phase water 
spectrum in comparison to ice a substantial change in geometry causing much smaller overlap in the excited state with the surrounding molecules is required.

Another important aspect which is different in the water and methane ices in comparison to the gas phase spectra is the lack of a clear onset of the ionization continuum. In the gas phase spectra we observe the Rydberg series converging to the ionization limit. However, in the condensed phases all the electronic states form a band structure according to the crystal potential. No vacuum level exists inside the condensed phase and an excited electron will only adopt a free electron wave nature when it has escaped the solid. This is typically what is monitored in photoelectron spectroscopy and described as a three-step model with excitation, transport to the surface and escape into vacuum (77). In XAS we only deal with the first step and therefore the ionization potential limit is not appropriate for understanding a condensed phase XAS spectrum. This situation could, however, be different on the surface where a molecule is both part of the condensed phase and connected to the outside vacuum.

\section{5 Symmetry properties of excited states in ice and water}

There is one particularly unique property of importance for XAS applied to water which is related to the dipole selection rule and thereby to the amount of $\mathrm{O} 2 p$ character in the excited orbitals. The hybridization between $\mathrm{O} 2 s$ and $\mathrm{O} 2 p$ depends strongly on the local symmetry. Let us first review the spectral intensities of the first excited states in the series of small molecules of the $\mathrm{C}, \mathrm{N}, \mathrm{O}$ and $\mathrm{F}$ hydride series where the molecular symmetry changes from 3-dimensional tetrahedral to linear.

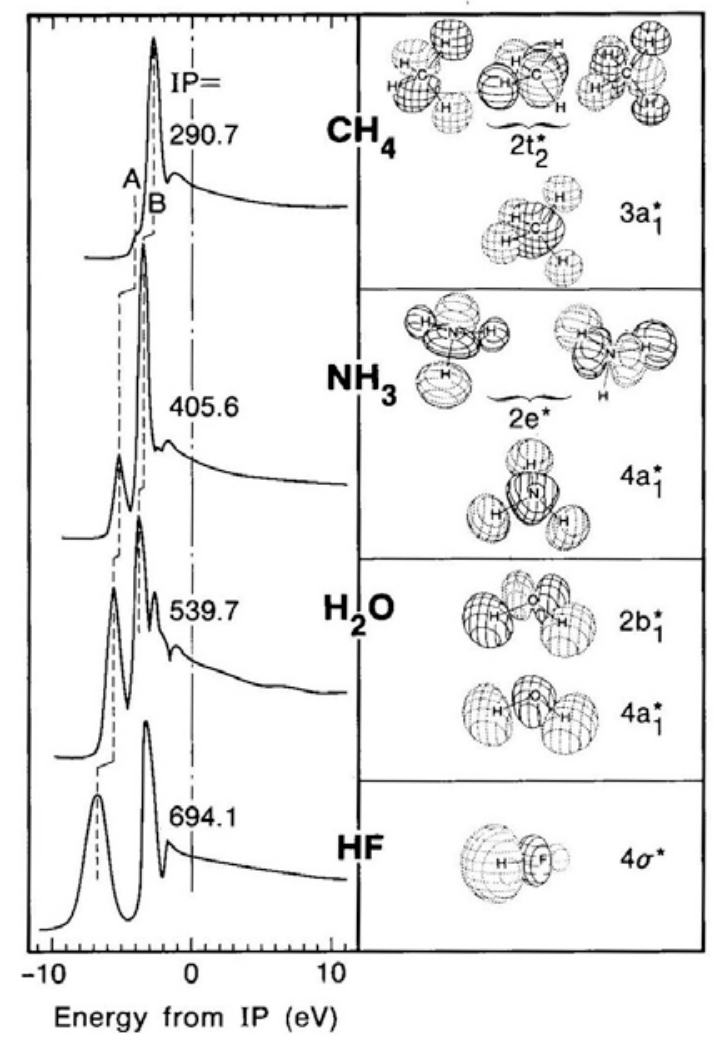

Fig. 14 Spectra of the isoelectronic molecules methane, ammonia, water and hydrogen fluoride together with the two first unoccupied molecular orbitals (40). The spectra have been aligned according to the ionization potential (IP). 
Fig. 14 shows spectra of the isoelectronic gas phase molecules methane, ammonia, water and hydrogen fluoride (40). The two lowest unoccupied molecular orbitals (LUMO) are rather similar to the $4 a_{1}$ and $2 b_{2}$ in water but with some most interesting trends with respect to the atomic hybridization as shown in the right part of fig. 14 (40). The $\mathrm{CH}_{4}$ molecule has tetrahedral $\mathrm{T}_{\mathrm{d}}$ symmetry which causes the carbon contribution to the LUMO $3 a_{1}$ orbital to be of pure C $2 s$ character whereas the contributions to the three degenerate LUMO+1 $2 \mathrm{t}_{2}$ orbitals are only of $\mathrm{C} 2 p_{\mathrm{x}}, \mathrm{C} 2 p_{\mathrm{y}}$ and $\mathrm{C} 2 p_{\mathrm{z}}$ characters. Since the dipole selection rule with an excitation from the $\mathrm{C} 1 s$ level only gives significant intensity for promotion of the electron to orbitals of carbon $2 p$ character we expect no intensity for the LUMO orbital but high intensity for the LUMO+1 orbitals. If we inspect the methane spectrum in fig 14 we only see a weak shoulder for excitation into the LUMO $3 a_{1}$ orbital and an intense peak for the LUMO $+12 \mathrm{t}_{2}$ orbitals. This follows perfectly the dipole selection rule as expected. If we look at the high resolution gas phase spectrum of methane in fig. 13 (74) we can still observe some very weak intensity related to excitations into the $3 a_{1}$ orbital. The reason for this is related to the Franck-Condon principle where the instantaneous geometry is seen as frozen on the time-scale of the excitation process and the molecular deformation along the coordinate of asymmetrical $\mathrm{C}-\mathrm{H}$ vibrations dynamically breaks the molecular tetrahedral $\mathrm{T}_{\mathrm{d}}$ symmetry. When a hydrogen atom is removed, as in $\mathrm{NH}_{3}$, the tetrahedral symmetry is broken and $2 p$ components are now allowed in the $a_{1}$ orbital providing intensity in the XAS spectrum. This trend is further strengthened as we remove one more hydrogen atom as in $\mathrm{H}_{2} \mathrm{O}$ and then one more for $\mathrm{HF}$. It looks like we have a most interesting sensitivity to detect tetrahedral symmetry through the atomic hybridization in the LUMO state. Could this also be applicable to ice where we form a semi-tetrahedral structure through $\mathrm{H}$ bonding?

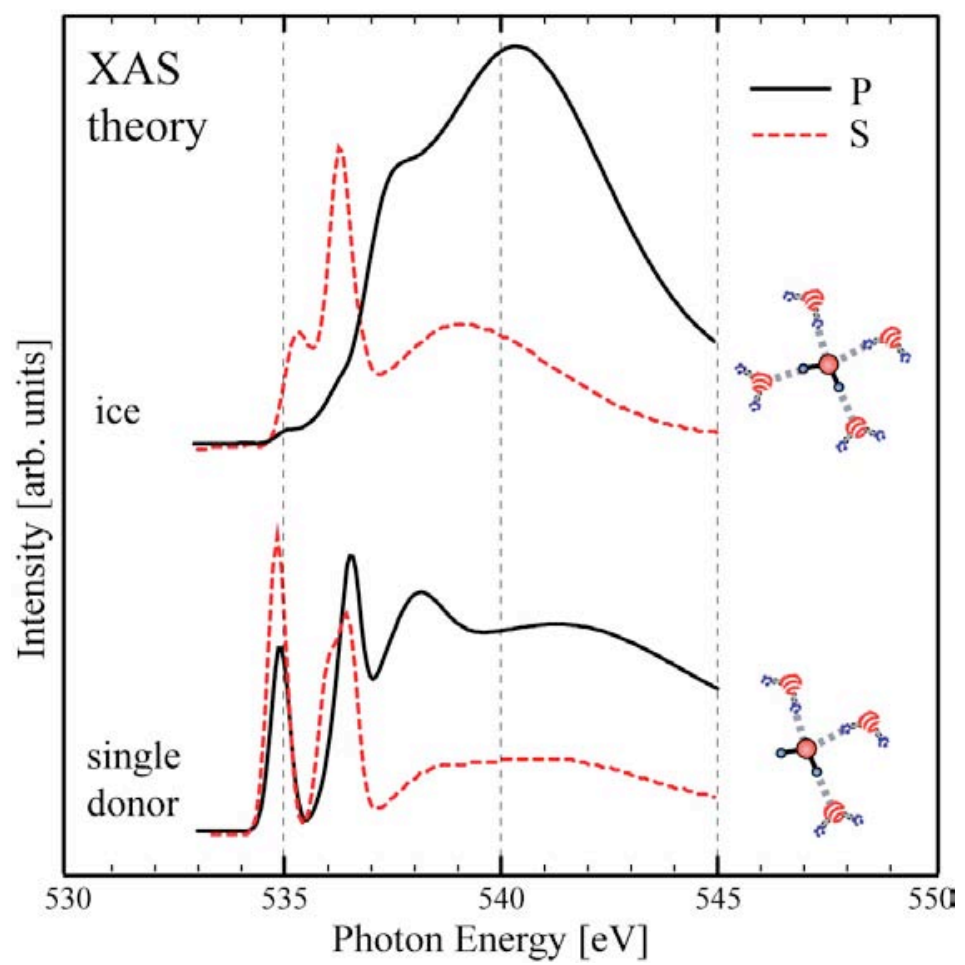

Fig. 15 Computed core excitation processes for bulk ice and a single-donor (SD) species from a liquid simulation (11). Separate contributions from $p$ character and location of $s$ states are indicated. Note that the intensity scale between $p$ and $s$ states is arbitrary. 
Such an hypothesis can be tested using theoretical calculations of the XAS spectra with the dipole operator to obtain the $p$-character and a simple, spherically symmetric $\mathrm{r}^{2}$ operator to detect $s$-character (11). The top part of fig. 15 shows the computed core excitation process using the two different transition matrix elements applied to a model of ice $\mathrm{I}_{\mathrm{h}}$ (11). We note that the electronic states with $s$ character reside close to the onset of the unoccupied states which we can denote LUMO. In this spectral region the $p$ character is extremely low but it is instead dominating in the post-edge region around $540 \mathrm{eV}$. This electronic structure situation resembles the methane molecule where the $s$ and $p$ atomic contributions are clearly separated into different excited states as a consequence of the tetrahedral symmetry. If we now start to deviate from the tetrahedral symmetry in ice by breaking $\mathrm{H}$-bonds we expect to see similar changes in the spectrum as between $\mathrm{CH}_{4}$ and $\mathrm{NH}_{3}$. The bottom part of fig. 15 shows the $s$ and $p$ contributions to the core-excited electronic structure for a particular SD species taken from an MD simulation(11). In this case we have broken the tetrahedral symmetry and now $p$ states can hybridize with the $s$ states. The lowest unoccupied states are now visible in the computed XAS spectrum. This results in the important pre-edge feature as seen in the water spectrum and the $p$ hybridization provides the intensity. The pre-edge feature will mainly be of $s$ character and the more distorted the local geometry is through weakening one of the donating H-bonds the more $p$ character can be involved in the hybridization.

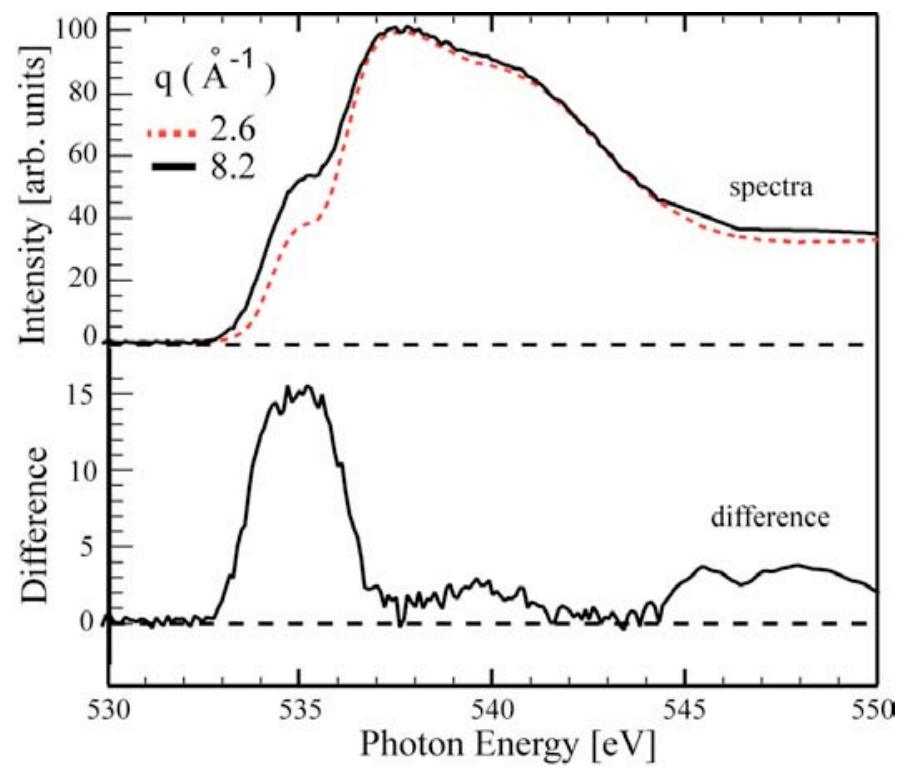

Fig. 16 (top) XRS spectra of water at different $q$-transfer and (bottom) the difference between the two spectra.

With XRS we have the ability to change the selection rule using different $q$-transfer and thereby probe also the $s$-character through non-dipole contributions. Fig. 16 shows XRS spectra for room temperature water at two different $q$-transfers. The spectra indicate that non-dipole contributions at the $\mathrm{O} \mathrm{K}$-edge of water can be observed for $q>4 \AA^{-1}$ (corresponding to $q r>$ $0.27)$ through an enhancement of the pre-edge intensity. Thus comparisons between XRS and XAS have to take these potential contributions into account. (Note that the XRS spectrum shown in Fig. 6 was measured with a momentum transfer $\left.q=2.6 \pm 1 \AA^{-1}\right)$. Also shown in Fig. 16 is the difference spectrum between the highest and lowest measured $q$ values in water and we clearly 
see a peak at the pre-edge. The difference spectrum should be dominated by the $s$ contribution in the pre-edge feature. This is an experimental confirmation of the symmetry properties of the preedge peak that it is dominated by $s$-character and breaking the semi-tetrahedral symmetry allows mixing with more $p$-character. We should however, note that using extremely high $q$-transfer would make the pre-edge feature less sensitive to the surrounding network since it will then both probe $s$ and $p$ character and would thereby not give rise to spectral intensity modifications through small changes in the $s p$ hybridization of the excited state.

\section{6 Electron localization in the core-excited states}

We can access the various resonances in the XAS spectra using selective excitation and then follow the subsequent core hole decay process. This can give information about how the excited state evolves in time due to electron rearrangements and changes in the nuclear coordinates. As discussed in section III.3 the excitation process occurs on an ultrafast time-scale of a few as and the $\mathrm{O} 1 s$ core hole has a life time of around 3.6 $f_{s}(78)$ which is determined by the rates of the two decay channels, Auger emission and fluorescence. If electron or nuclear rearrangements occur during the lifetime of the core hole state it will show up as a signature in the decay spectra. With the knowledge of the core hole life time, the time scales of the electron or nuclear rearrangements due to the core excitation can be determined $(20 ; 33 ; 79-88)$. In the following we will concentrate on the electron localization-delocalization processes in water as measured by using resonant excitation into the pre-edge and post-edge regions.

Fig. 17 shows the Auger decay process upon resonant excitation into either a specific resonance in the XAS spectrum or at high excitation energy. The latter results in a free-electronlike state which delocalizes extremely fast away from the excited atom; eventually, if the electron is transferred to the surface without losses, it escapes resulting in ionization. This is denoted non-resonant excitation and is similar to excitation in the gas phase well above the ionization threshold. The following Auger decay shown in fig. 17 as A-C is therefore called "normal Auger decay" and results in a two-valence-hole final state. The core electron can alternatively be excited to a specific resonance in the XAS spectrum, denoted resonant excitation. If the excited state is spatially localized at the excited molecule there is a chance that the electron is captured for longer times. This can be observed through large differences in the Auger spectra if the excited electron is still present or already delocalized when the core-hole decays. If the excited electron delocalizes faster than the core-hole decay process, it gives the same final state as normal Auger decay as shown in fig. 17 as B-C. On the other hand, an excited electron that remains localized during the core-hole decay process affects the kinetic energy of the Auger electron causing what is called a spectator shift; this decay channel is denoted "spectator Auger decay" and is shown in fig 17 B-D.

Here we consider the O KLL Auger decay, where L corresponds to molecular orbitals of O2p character $\left(1 b_{1}, 3 a_{1}\right.$ and $1 b_{2}$ in the water molecule). Fig. 17E illustrates the kinetic energy shift of the outgoing Auger electron as given by two extreme cases in simulated spectra (20) for a water tetramer with two electrons removed from the L shell: One spectrum with the excited electron completely removed (normal Auger decay) and one with the excited electron localized in the LUMO orbital (spectator Auger decay). The spectator Auger spectrum is shifted approximately 5 $\mathrm{eV}$ towards higher kinetic energy relative to the normal Auger spectrum, reflecting a general spectral trend of spectator decay towards higher kinetic energy due to the screening of the final state by the localized (spectator) electron. From the intensity ratio of the two final states and the life-time of the core hole the electron delocalization rate can be determined (79). 

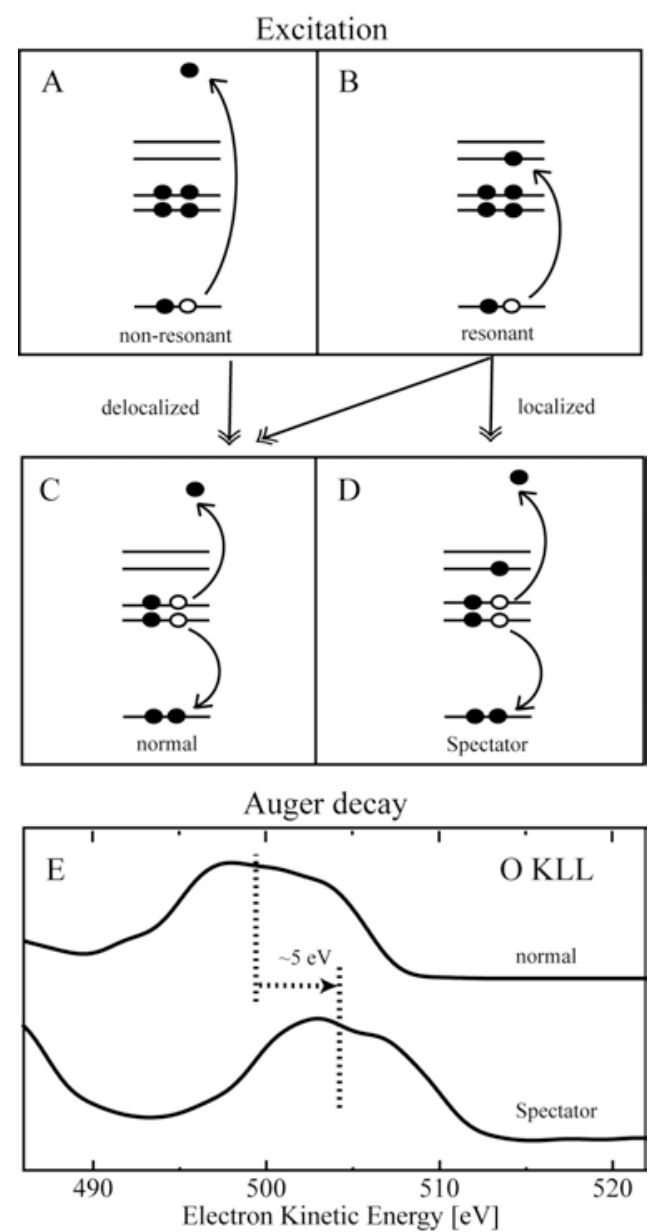

Fig. 17 Schematic representation of the core excitation and deexcitation processes. An O1s electron is (A) ionized and (B) pumped to an unoccupied molecular orbital producing the excited electron and the core hole. (middle) (C) During the life-time of the core hole there is a probability for the excited electron to be transferred to another atomic site through a delocalization process. (bottom) With a decay time of $3.6 f s$, the Auger process fills the core-hole with one electron from the valence band and a second electron takes up the excess energy and is emitted. Two different final states can be observed, (D) normal Auger where the excited electron is missing on the decaying atom and (E) spectator Auger where the excited electron is still present, i.e. the excited electron remains localized. In the spectator case (E) the ejected electron will have different kinetic energy due to the screening of the final state by the additional localized electron.

In fig. 18, the measured O KLL Auger decay spectra for selected excitation energies in liquid water are shown (20). The inset shows the corresponding XAS spectrum with arrows indicating excitation energies. Upon excitation at the pre-edge $(535 \mathrm{eV})$, the spectator Auger intensity (center around $506 \mathrm{eV}$ ) is pronounced, whereas the normal Auger intensity (centered around 501 $\mathrm{eV}$ ) is very small. Upon excitation to the post-edge, it is the other way around, with normal Auger intensity dominating the spectra. Thus, for core excitations into the pre-edge state, associated with the weakly $\mathrm{H}$-bonded $\mathrm{OH}$ groups in $\mathrm{SD}$ species in the liquid, the electron remains localized far longer than the core-hole life-time. For excitations into the post-edge state, associated with $\mathrm{OH}$ groups involved in strong $\mathrm{H}$ bonds, the delocalization rate is instead much faster than the core-hole life-time. The delocalization rate, $\tau_{\mathrm{CT}}$, was determined from the experiment by scaled subtraction of the relative intensity of normal Auger decay $\left(f_{\text {aug }}\right)$ using the 


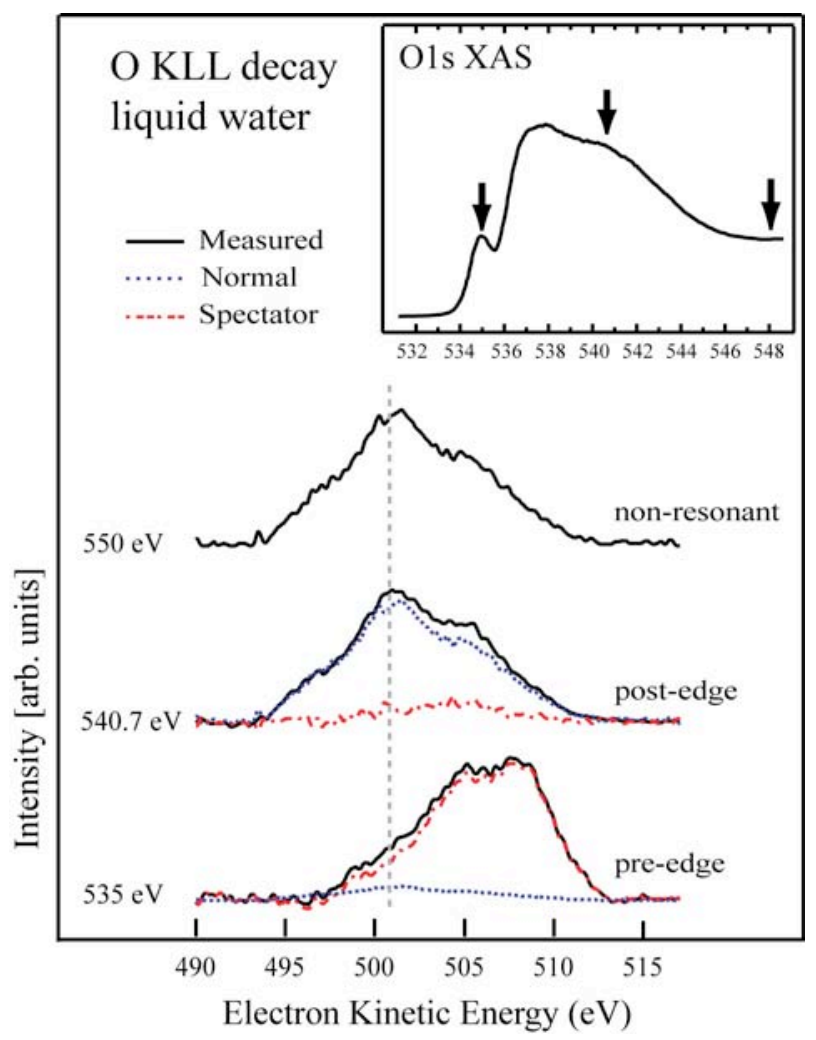

Fig. 18. Resonant Auger spectra of water with selective excitation as either non-resonant $(550 \mathrm{eV})$ or to the pre-edge and post-edge resonances in the XAS spectrum (insert) (20). Using the decay from non-resonant excitation as model spectrum for normal Auger decay, the normal Auger fraction $\mathrm{f}_{\text {aug }}$ is quantified for the excitation at the post-edge and at the pre-edge. The difference between the measured spectra (line) and the normal Auger fraction (dotted) is associated with the spectator decay spectra (dashed), shifted to $\sim 5 \mathrm{eV}$ higher kinetic energy in agreement with theory (see fig. 17). All spectra were area normalized in the region $495-510 \mathrm{eV}$ prior to the quantification.

measured non-resonant Auger spectrum at $550 \mathrm{eV}$ to describe the normal Auger spectral shape (dotted in fig. 18). Applying the relation $\tau_{\mathrm{CT}}=\tau_{\mathrm{c}} *\left(\mathrm{f}_{\text {aug }}{ }^{-1}-1\right)$, where $\tau_{\mathrm{c}}$ is the $\mathrm{O} 1 s$ core-hole lifetime, $\tau_{\mathrm{CT}}$ is determined to be faster than 500 as for post-edge excitation (normal Auger fraction $\mathrm{f}_{\text {aug }}>$ 0.88 ), and slower than $20 f_{s}$ for pre-edge excitation $\left(f_{\text {aug }}<0.15\right)$. Similar results have also been obtained for ice where the post-edge is connected to excited states in the bulk and the pre-edge to the surface (20).

We can also use the other decay channel involving fluorescence which is also denoted x-ray emission (XES) $(13 ; 14 ; 25 ; 34 ; 37 ; 89)$. It has been shown by Tokushima et al. and Fuchs et al. that the lone pair $1 b_{1}$ peak is split into two components, which Tokushima et al. interpreted (25; $37)$ as due to tetrahedral $\left(1 b_{1}^{\prime}\right)$ and $H$-bond distorted $\left(1 b_{1} "\right)$ structures in the liquid which coexist in a fluctuating H-bonding network (38). We will discuss the implications of the two peaks for the emerging picture of water structures in section V.1. Similar to the case of resonant Auger above we can follow how the XES spectra change as we excite into specific resonances in the XAS spectrum. It has been shown that upon post-edge excitation there is no shift of the energy positions of the spectral features $(25 ; 37)$ with respect to non-resonant excitation at $550 \mathrm{eV}$, which is fully consistent with the resonant Auger results in fig. 18 (20). However, upon postedge excitation the intensity of the $1 b_{1}{ }^{\prime}$ peak, connected to tetrahedral structures, is enhanced 
reflecting that this resonance is connected to strong H-bonding. Fig. 19 shows the evolution of the $1 b_{1}$ XES spectra for $\mathrm{D}_{2} \mathrm{O}$ at excitation energies close to the XAS pre-edge $(25 ; 37)$. We note that the $1 b_{1}$ " shifts towards lower energies as we decrease the excitation energy towards the preedge whereas the $1 b_{1}{ }^{\prime}$ is at constant energy and diminishes in intensity. This is fully consistent with the assignments of both the $1 b_{1}$ " and pre-edge spectral features as being H-bond distorted or even as SD species. We also observe the spectator shift of the $1 b_{1}$ " upon pre-edge excitation in full agreement with the resonant Auger results in fig. 18. However, in the case of XES the spectator shift is towards lower emission energies whereas in the Auger case it is towards higher kinetic energies. This can be understood by considering the number of electrons involved in the respective decay process. The spectator shift in the Auger decay arises due to the more efficient screening by the spectator electron of the two valence holes in comparison to screening the single core hole state which lowers the final state energy and thereby increases the energy released in the decay process. In the x-ray emission process both the initial state and final state contain only a single hole and the screening of the spectator electron is more energy efficient for the deeper-lying core hole than for the valence hole causing greater stabilization of the initial state and thereby a decrease of the emission energy.

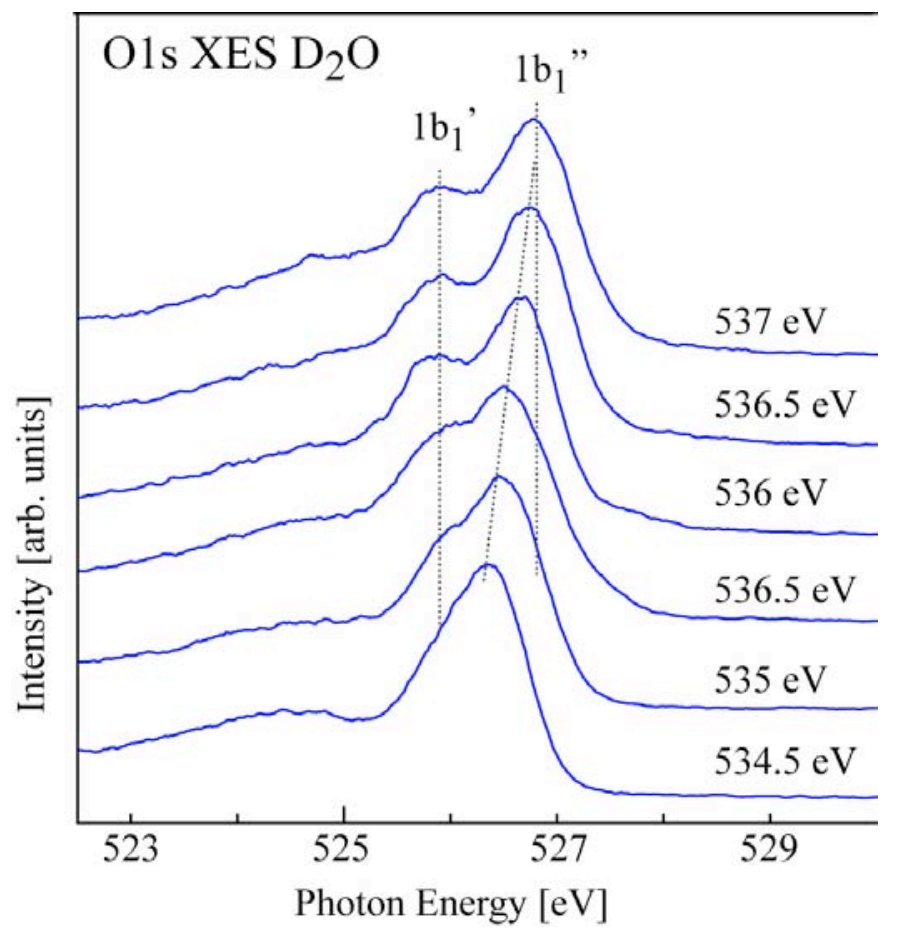

Fig. 19 Excitation energy dependence of $\mathrm{D}_{2} \mathrm{O}$ XES spectra from 537 to $534.5 \mathrm{eV}$ demonstrating the Raman shift of the $1 b_{1}$ " peak (25).

Can we understand why the pre-edge is a strongly localized state whereas the post-edge is delocalized? One obvious difference is the higher energy of the post-edge which resides in the energy regime above the gas phase ionization potential whereas the pre-edge is at threshold and could have significant core excitonic character. Let us turn to some theoretical calculations of the wave functions of these two O1s excited states. In fig. 20, we show representative orbital plots for typical pre-edge and post-edge excited state wave functions, computed using DFT (28) on 
cluster models from an MD snap-shot (20). The post-edge (fig. 20a) is dominated by states delocalized along the H-bonds over many atomic centers. This is consistent with the large fraction of normal Auger decay at this excitation energy, showing that the excited electron is propagated into the H-bonded network at the time of the core-hole decay. In contrast to this, the orbital corresponding to the pre-edge excitation for a typical SD configuration (fig. 20b) is localized along the internal non-H-bonded $\mathrm{O}-\mathrm{H}$ bond (3). The confinement of the wave function causes a large difference in localization character of the pre-edge excited state in the liquid compared to the conduction band in both water and ice.

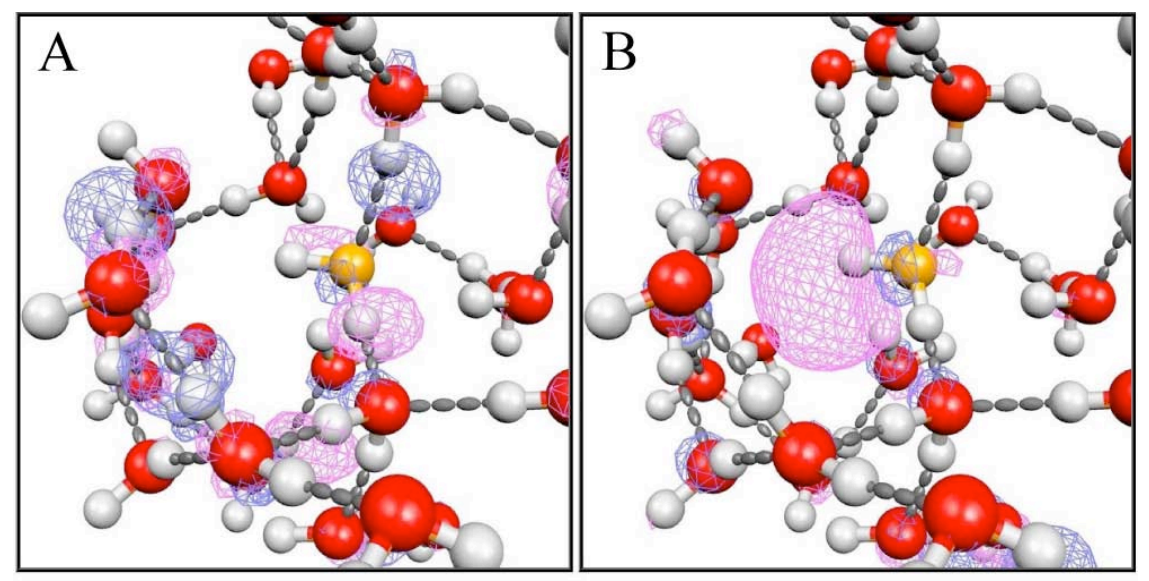

Fig. 20 Orbital plots of core-excited states in (a) the post-edge region and (b) the pre-edge regions with the core hole on an SD species with the structure taken from a snap-shot of an MD simulation (20).

These results are fully consistent with the interpretation that the post-edge spectral feature is directly connected to strong $\mathrm{H}$-bonding whereas the pre-edge feature is connected to structures that create a cavity around the $\mathrm{OH}$ where the excited electron can become fully localized without any significant overlap with the surrounding. If we then want to call the pre-edge a localized Frenkel excitation and the post-edge a delocalized Wannier excitation, as in Tse et al. (26), is only a question of semantics.

\section{7 Shape resonances and bond length with a ruler}

There is an important concept in XAS denoted "bond-length-with-a-ruler" which was developed by Stöhr and coworkers regarding shape resonances which appear in the ionization continuum in spectra of free and adsorbed molecules $(40 ; 90-92)$ as discussed in section III.2. As shown in fig. 10 there is no real shape resonance in the continuum in the free water molecule spectrum whereas many molecules such as $\mathrm{N}_{2}, \mathrm{O}_{2}, \mathrm{CO}, \mathrm{C}_{2} \mathrm{H}_{2}, \mathrm{C}_{2} \mathrm{H}_{4}$ etc. show a clear resonance (40). These resonances are often of $\sigma^{*}$ symmetry with antibonding character between the connecting atoms. Excitations into the antibonding $\mathrm{OH}$ orbitals, denoted $4 \mathrm{a}_{1}$ and $2 \mathrm{~b}_{2}$, appear in the case of the free water molecule in the XAS spectrum in fig. 10 as bound states below the ionization potential. The question is if the post-edge resonance in condensed phase could mimic in some ways the shape resonance in small molecules. 

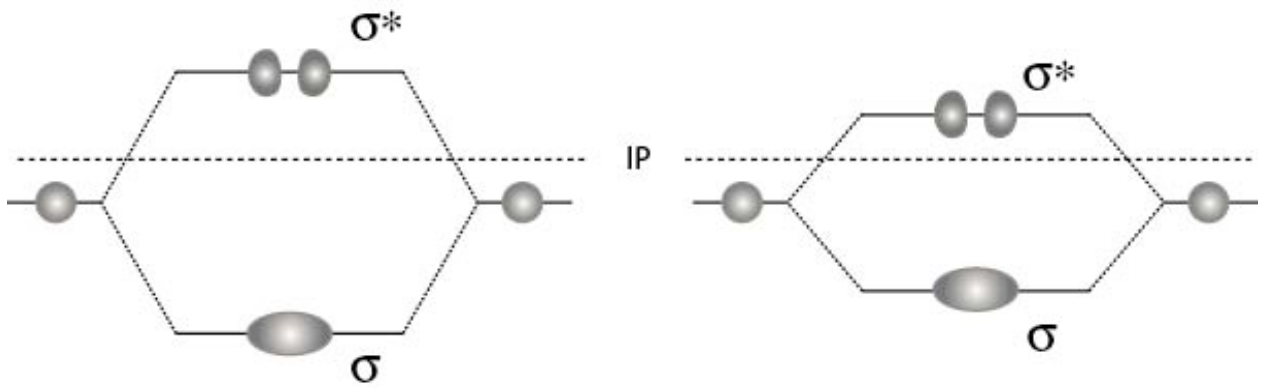

Fig. 21 Illustration of the relationship between the bonding-antibonding splitting and the energy position of a $\sigma^{*}$ peak relative to the ionization potential (IP). The left figure depicts shorter bond length compared to the right figure (larger splitting), which places the $\sigma^{*}$ orbital higher in energy relative to the IP.

The $\sigma^{*}$ shape resonance above or near the ionization potential (IP) appears when the excited core electron is briefly trapped in a molecular potential barrier before leaving the system. A molecular orbital picture of this phenomenon describes a process in which the electron is excited into an unoccupied antibonding molecular orbital above the ionization potential before it is ejected as a photoelectron. The energy position of the $\sigma^{*}$ resonance is, therefore, dependent on the splitting between bonding and antibonding orbitals, as depicted in fig. 21 , which, in turn, is correlated with the bond length, since the $\sigma^{*}$ orbital is located along the intermolecular axis. A smaller splitting, which results from a longer bond length, positions the $\sigma^{*}$ orbital at a lower energy relative to the IP, while a larger splitting (shorter bond length) results in the $\sigma^{*}$ position at a higher energy. Sette et al. empirically found a linear correlation between $\sigma^{*}$ resonance position relative to the IP and the bond length that can be used to predict unknown bond lengths with a high precision (90).
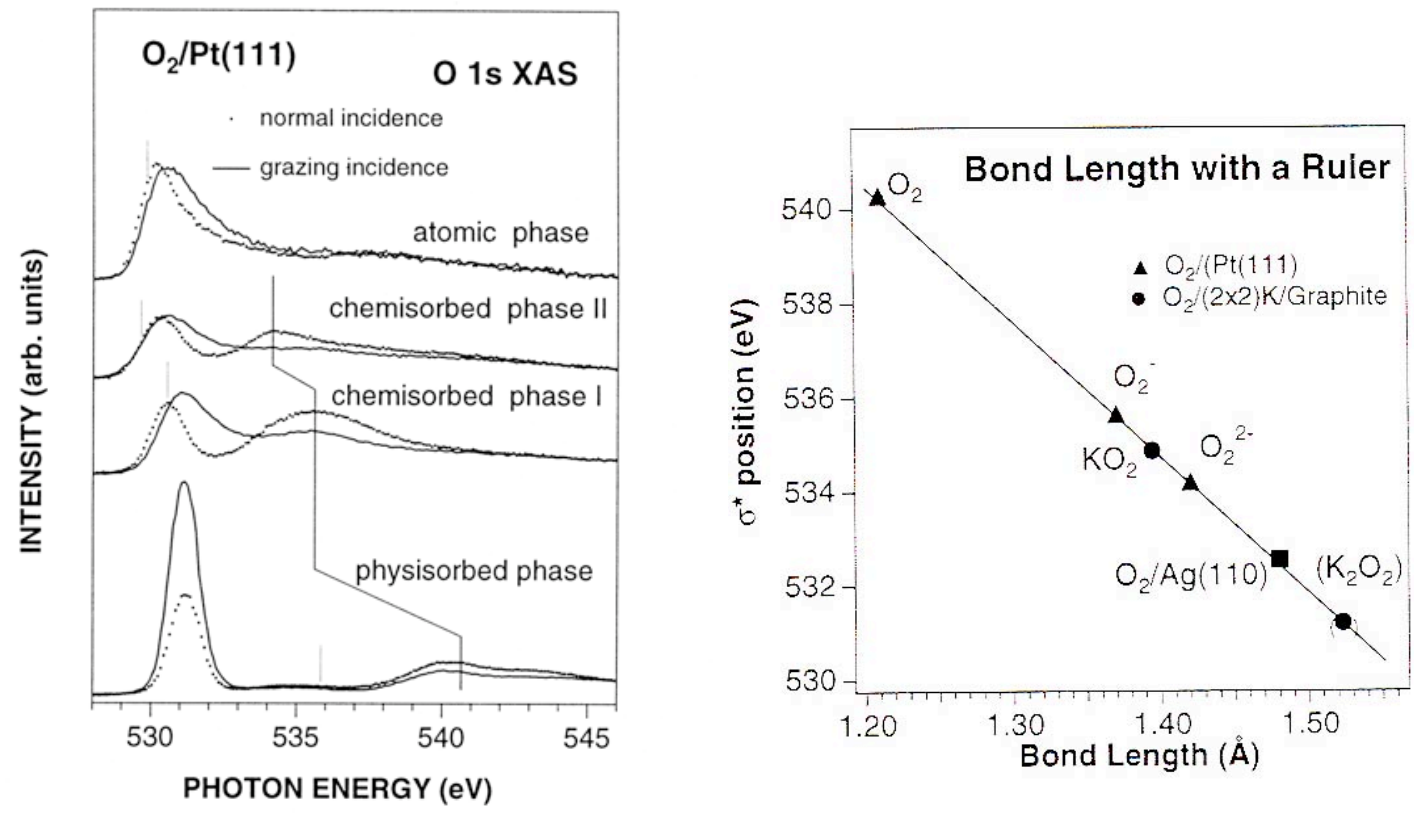

Fig. 22 . Left: $\mathrm{O} 1 s$ XAS spectra of $\mathrm{O}_{2}$ on $\mathrm{Pt}(111)$ in the different adsorption phases: physisorbed (25K), first chemisorbed phase $(90 \mathrm{~K})$, second chemisorbed phase $(135 \mathrm{~K})$ and dissociated atomic $(2 \times 2)$ ordered phase (room temperature) (94). Right: $\sigma^{*}$ position for the different adsorption species of $\mathrm{O}_{2}$ on $\operatorname{Pt}(111)$ (triangular markers) and other substrates as a function of bond-length showing linear correlation (93). 
The bond-length-with-a-ruler model has been used to characterize the different species of many molecular adsorbates on surfaces (40) but here we demonstrate the principle for the oxygen molecule $\left(\mathrm{O}_{2}\right)$ adsorbed on $\mathrm{Pt}(111)$ (93). Fig. 22 (left) $(93 ; 94)$ shows the O1s XAS spectra of $\mathrm{O}_{2}$ on $\mathrm{Pt}(111)$ at different substrate temperatures. As the substrate temperature is increased from $25 \mathrm{~K}$ (physisorbed phase) to $90 \mathrm{~K}$ (first chemisorbed phase) and to $135 \mathrm{~K}$ (second chemisorbed phase), the $\sigma^{*}$ resonance peak shows a downward shift as indicated by the vertical lines, suggesting that the $\mathrm{O}-\mathrm{O}$ bond is progressively lengthened as the temperature is raised. It can be concluded that the oxygen molecules are in the superoxo $\left(\mathrm{O}_{2}^{-}\right)$and peroxo $\left(\mathrm{O}_{2}{ }^{2-}\right)$ configurations in the first and second chemisorbed phases, respectively (93; 94). In fig. 22 (right) (93), the triangular markers show that the position of $\sigma^{*}$ resonance and bond length are linearly correlated, confirming the conclusion of Sette et al. (90)

In the case of water, the post-edge can be assigned to a shape resonance analogous to adsorbed $\mathrm{O}_{2}$ on $\mathrm{Pt}(111)$. It has been discussed in previous sections that the post-edge peak of water and ice arises from strong hydrogen-bonding between two water molecules, which is described as a result of orbital mixing in addition to strong dipole-dipole interaction (94). Therefore, the energy position of this peak depends on the H-bond distance when a core electron is excited into this high-lying, unoccupied mixed orbital. Furthermore, fig. 20 shows the computed wave function corresponding to a post-edge excited electron where it is clearly of antibonding character between the water molecules. Fig. 23 shows simulated XAS spectra of ice clusters as function of donating H-bond (oxygen-oxygen) distance (6). The energy position of the post-edge peak is shown to be dependent on $\mathrm{O}-\mathrm{O}$ distance in a roughly linear correlation with a factor of $8 \mathrm{eV} / \AA$ (6).

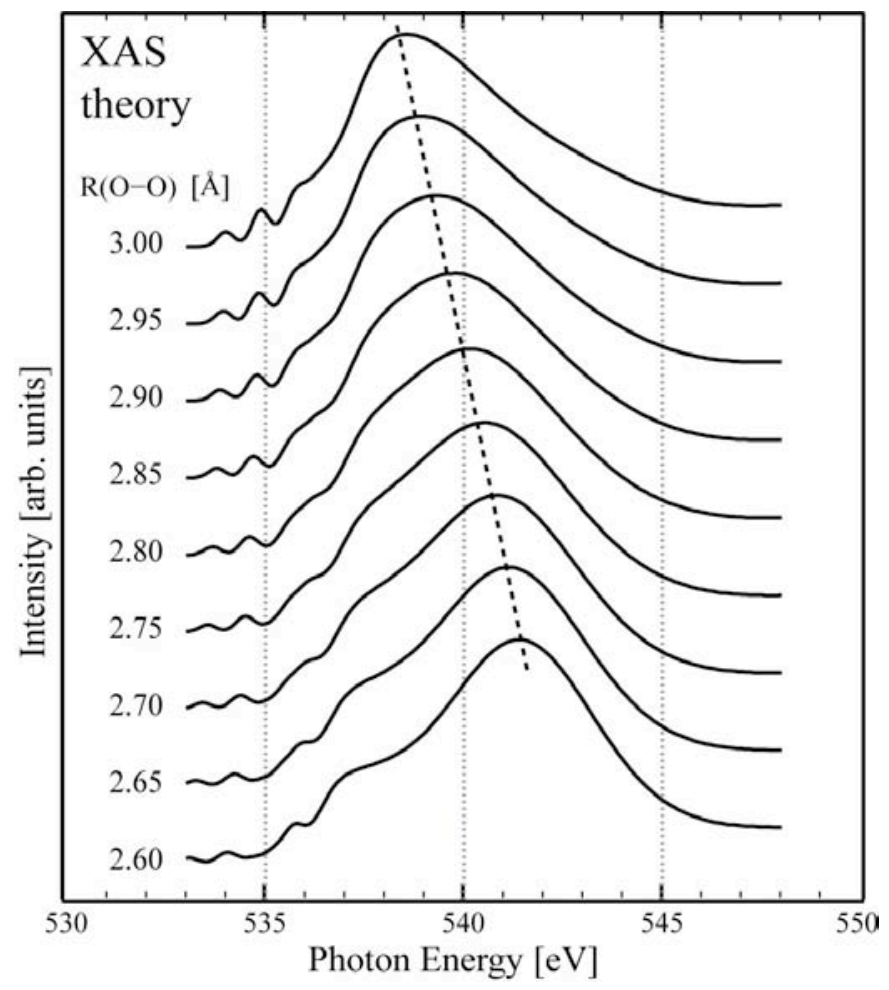

Fig. 23. Simulated XAS spectra for 17 molecule cluster (ice structure) as a function of donating H-bond (O-O) distance for both bonds (6). 
Experimental evidence for the bond-length-with-a-ruler concept in liquid water is presented in a study by Cavalleri et al. where the XAS spectra of $\mathrm{HCl}$ aqueous solutions at various concentrations were measured using FY (96). Fig. 24 shows the O $1 s$ FY-XAS spectra of $0.1,1$, 4 , and $6 \mathrm{M} \mathrm{HCl}$ solutions compared with the spectrum of pure liquid water, all normalized at 550 $\mathrm{eV}$ (96). The pre- and main-edge peaks increase at low $\mathrm{HCl}$ concentration $(0.1$ and $1 \mathrm{M})$ due to a decrease in the average number of donating H-bonds per molecule compared to pure liquid water; the post-edge, however, is relatively unchanged. At high concentrations, the opposite change is seen in the pre- and main-edge, which are decreased compared to pure liquid water, while the post-edge region increases and the post-edge peak shifts in energy position from 540$541 \mathrm{eV}$ for $0.1 \mathrm{M}$ to $541-542 \mathrm{eV}$ for $6 \mathrm{M}$.

The spectral differences between low and high concentrations of $\mathrm{HCl}$ in water can be described in terms of varying fractions of the Eigen $\left(\mathrm{H}_{3} \mathrm{O}^{+}\right)$and $\mathrm{Zundel}\left(\mathrm{H}_{5} \mathrm{O}_{2}{ }^{+}\right)$structural motifs of protonated water. Analysis of the post-edge difference spectra for 1, 4, and $6 \mathrm{M} \mathrm{HCl}$ solutions (see Ref. (96) for details) has revealed that the Zundel species dominates at low concentrations while at high concentrations the Eigen form dominates. Within the framework of the bondlength-with-a-ruler model, the Eigen species, which has a shorter H-bond distance compared to the Zundel species (1.55 $\AA$ and $1.7 \AA$, respectively) is characterized by a higher energy of the post-edge, which is exactly what we observe in fig. 24 (96). Theoretical simulations also show an upward shift of $1 \mathrm{eV}$ of the post-edge for the Eigen species compared to the Zundel species and bulk water (96).

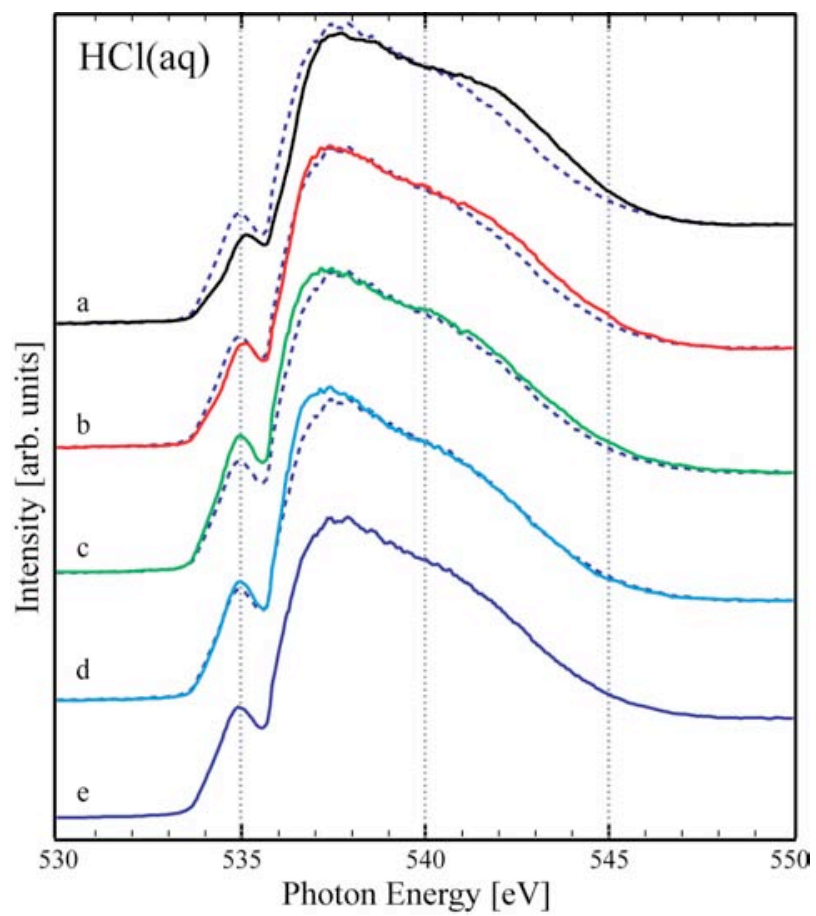

Fig. 24. O $1 s$ XAS spectra (fluorescence yield) of (a) $6 \mathrm{M} \mathrm{HCl(aq),} \mathrm{(b)} 4 \mathrm{M} \mathrm{HCl(aq),} \mathrm{(c)} 1 \mathrm{M} \mathrm{HCl(aq),} \mathrm{(d)} 0.1 \mathrm{M}$ $\mathrm{HCl}(\mathrm{aq})$, and (e) pure water (96). Dashed lines in (a-d) show the spectrum of pure water for comparison purposes. All spectra are intensity normalized at $550 \mathrm{eV}$.

The sensitivity of the post-edge position to H-bond distance can, therefore, be a powerful tool to investigate certain effects that modify the H-bonding network of water, such as ion hydration or temperature change. We would therefore expect for water models that maintain the local 
tetrahedral coordination and predict on average a small increase in H-bonding distances, to observe a shift in the post-edge towards lower energies when the temperature is increased. This is also seen in calculated XAS spectra of structures obtained from various MD simulations and will be discussed in section VI (6). This is in contrast to the measured XAS spectra as a function of temperature (fig. 2 and fig. 34).

\section{Various Ices and Adsorbed Overlayers}

Although the focus here is to understand the liquid water spectrum it is essential to derive an understanding of the spectral sensitivity to H-bond structures in general. In this section we will discuss various model systems such as bulk and surface of ice, water adsorbed on metal surfaces and the amino group in glycine adsorbed on $\mathrm{Cu}(110)$.

\section{IV.1 Surface and bulk ice}

Let us now take a closer look at the spectra of ice. We will first discuss ice prepared by condensation from the vapor phase onto metal single-crystal surfaces. It is known that ice forms a very corrugated structure where the first adsorbed monolayer remains exposed even for ice film thicknesses up to 50 layers (97-99). At low temperatures (below 130K) it is assumed that ice grows in an amorphous structure whereas at higher temperatures, close to the sublimation point, it is assumed that ice is in a crystalline form and resembles cubic ice $I_{c}(97 ; 98 ; 100)$. The amorphous ice film grows much more in a layer-by-layer fashion $(97 ; 98)$.

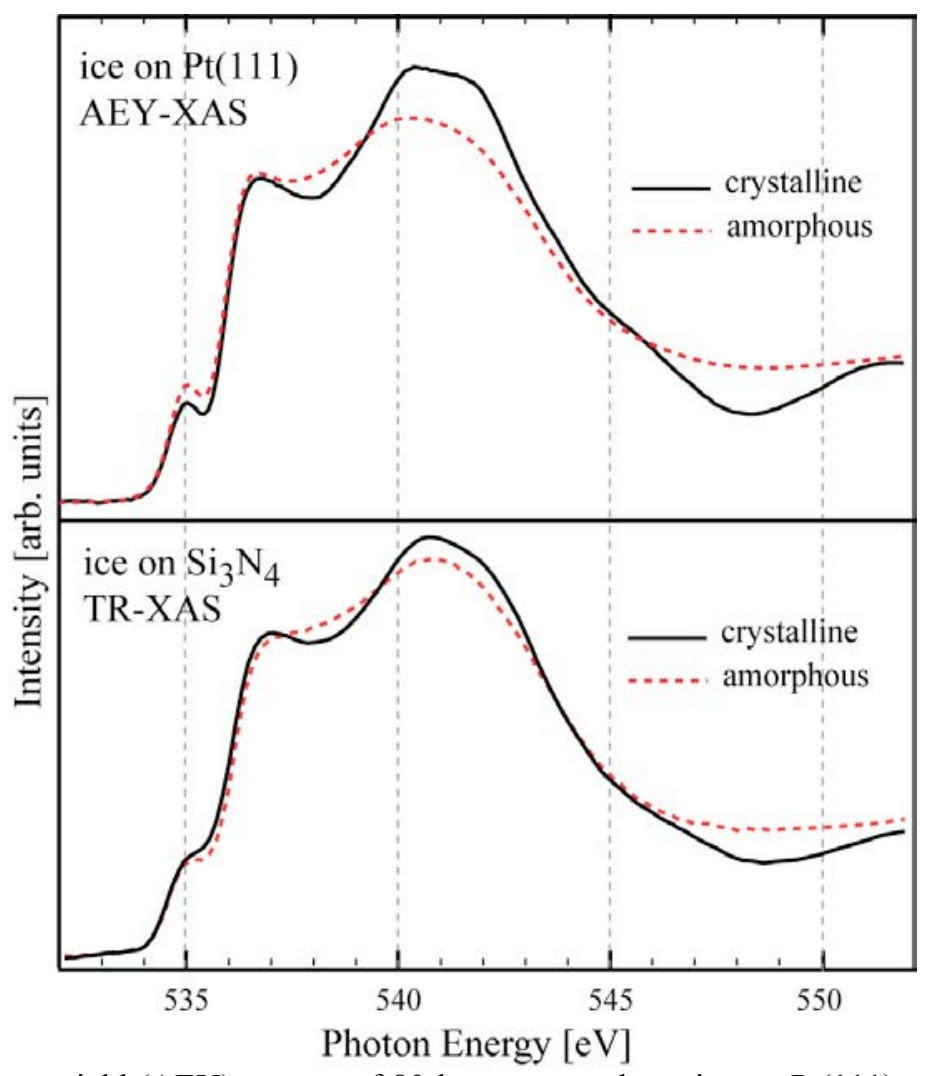

Fig. 25 Left: Auger electron yield (AEY) spectra of 80 layers amorphous ice on $\operatorname{Pt}(111)$ grown at $\sim 100 \mathrm{~K}$ (red) and 48 layers crystalline ice prepared by isothermal heating of amorphous ice at $\sim 150 \mathrm{~K}$ (black). Right: Transmission spectra of amorphous ice (red) and crystalline ice (black) on $\mathrm{Si}_{3} \mathrm{~N}_{4}$ membranes. All spectra have been normalized to the same area from 532 to $550 \mathrm{eV}$. 
Fig. 25 compares the spectrum of ice as prepared on $\operatorname{Pt}(111)$ at $80 \mathrm{~K}$ with the spectrum after the sample has been heated to the so-called crystallization temperature of $150 \mathrm{~K}(97 ; 98 ; 101$; 102); both spectra were measured with AEY with a detection depth of $20 \AA$ (30). The experimental details are described in (103). These spectra are rather similar to what has been observed previously with AEY $(31 ; 104)$. They are also similar to the amorphous and crystalline ice spectra measured in transmission mode shown in fig. 25 (105).

We note some major differences between the spectra of the two ice forms, however. The post-edge of crystalline ice is higher in intensity, more structured, and slightly shifted to higher energy. In addition, there is more fine-structure beyond the post-edge for crystalline ice which has been attributed to multiple-scattering resonances due to long-range order $(28 ; 31 ; 57)$. We can note that the pre-edge feature is more intense for the amorphous film in comparison to the crystalline ice $(97 ; 98)$. The broader post-edge in the low-temperature film is a direct consequence of disorder giving rise to a broader distribution of H-bond distances. Based on the concept of the "bond length with a ruler" (see section III.7) we would then assume a distribution of post-edge energies reflecting the distribution of $\mathrm{H}$-bond distances. The post-edge is furthermore broadened due to the internal $\mathrm{OH}$ stretch vibrational motion since the post-edge energy depends strongly on the internal $\mathrm{OH}$ distance (28) according to the Franck-Condon principle (see section III.3). However, the vibrational broadening from the internal modes will affect the two ice films in a similar fashion. We note that in spite of intense research the ice samples are poorly understood and we will discuss further below that these films can contain different mixtures of local amorphous structures.
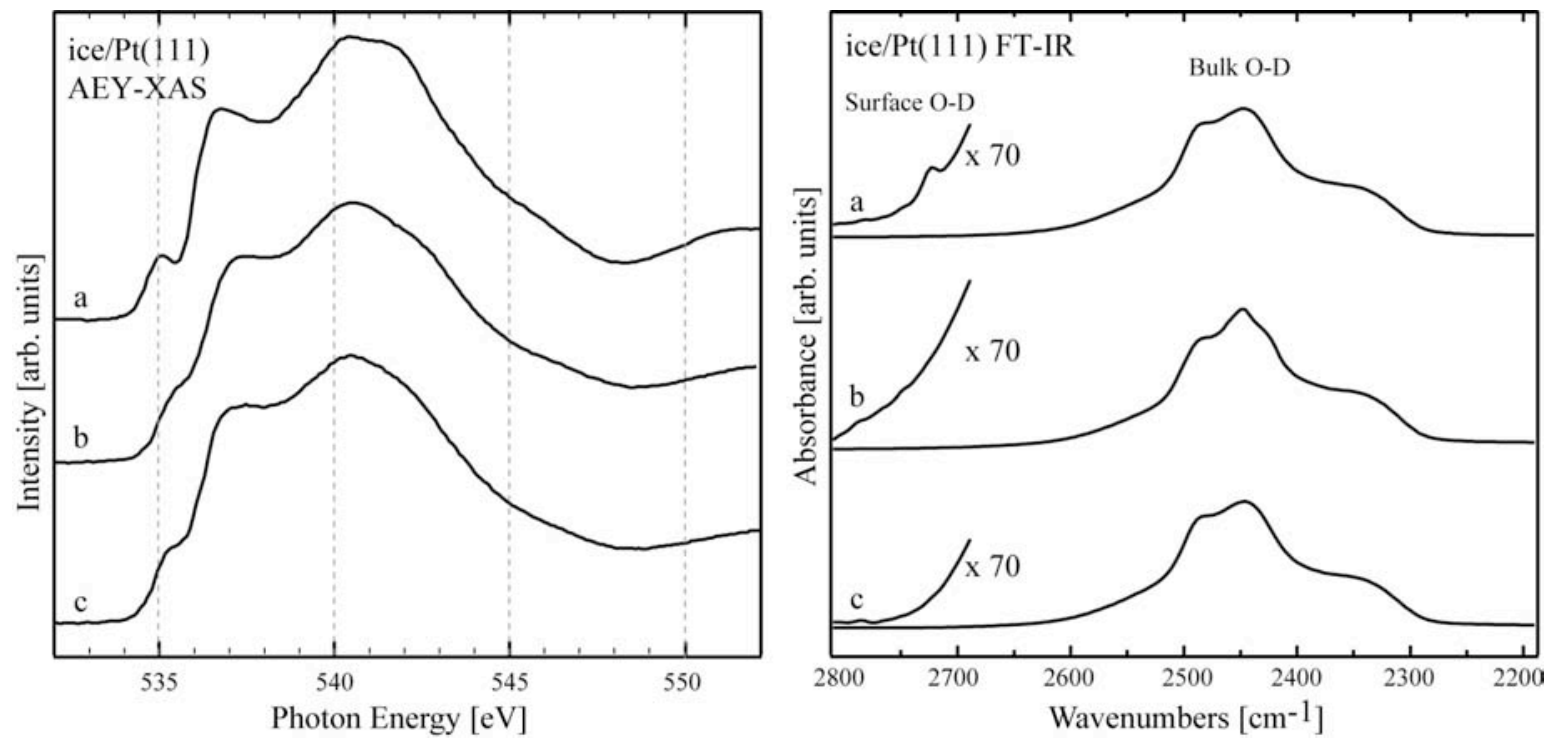

Fig. 26 AEY XAS (left) and IRAS (right) of (a) crystalline ice obtained by heating 80 layers of amorphous ice on $\operatorname{Pt}(111)$ to $150 \mathrm{~K}$ for 16 minutes, (b) crystalline ice covered with a multilayer of $\mathrm{NH}_{3}$, and (c) $\mathrm{NH}_{3}$-terminated crystalline ice after heating to $114 \mathrm{~K}$ for 5 minutes, leaving one layer of $\mathrm{NH}_{3}$ on the ice surface. The region of the surface O-D stretch in the IRAS spectra has been multiplied by a factor of 70.

In Wernet et al. the surface of ice was used to demonstrate the spectrum of an SD species at the surface of ice $(3 ; 30)$. Let us now revisit this question. Fig. 26 shows how the XAS spectra of crystalline $\mathrm{D}_{2} \mathrm{O}$ ice evolve after we deposit a few layers of ammonia and then subsequently 
anneal the film to desorb the ammonia multilayer in order for only a monolayer of ammonia to remain on top of the ice film. In the same setup (106) infrared absorption spectroscopy (IR) was also conducted in the $\mathrm{OH}$ stretch region of films prepared exactly the same way as for the XAS measurements. Let us focus on the free OD stretch seen as a small sharp feature at around 2700 $\mathrm{cm}^{-1}$ in the top spectrum of fig. 26; this peak has been identified as due to dangling free $\mathrm{OH}$ groups at the ice surface (107). This sharp feature in the clean crystalline ice film disappears upon ammonia adsorption onto the ice film. This means that we have saturated the dangling $\mathrm{OH}$ groups by H-bonding to ammonia molecules and we can now observe the consequences in the XAS spectrum. There are substantial changes in all spectral regions but in particular the sharp pre-edge feature seems to be quenched and only a shoulder is observed. Let us now take a detailed look at the surface sensitive spectrum obtained by Nordlund et al. on an ice film prepared using similar preparation techniques (30). The spectrum was obtained using AEY detection with the electron analyzer positioned such that emitted electrons were detected at extreme grazing angle to give a maximally surface-sensitive spectrum (30). Fig. 27 shows a curve-fitting of the spectra before and after adsorption of ammonia. We see that the pre-edge feature is not quenched, but shifted up in energy by $0.45 \mathrm{eV}$ and now resides at the onset of the main-edge. We also observe that the main-edge is less sharp and that there is an increase in the post-edge intensity after ammonia adsorption. We note that after adsorbing ammonia there is no residue of the pre-edge peak at the pre-edge energy position of the clean film. This must imply that all the pre-edge intensity in the clean film is related to dangling $\mathrm{OH}$ on the surface.

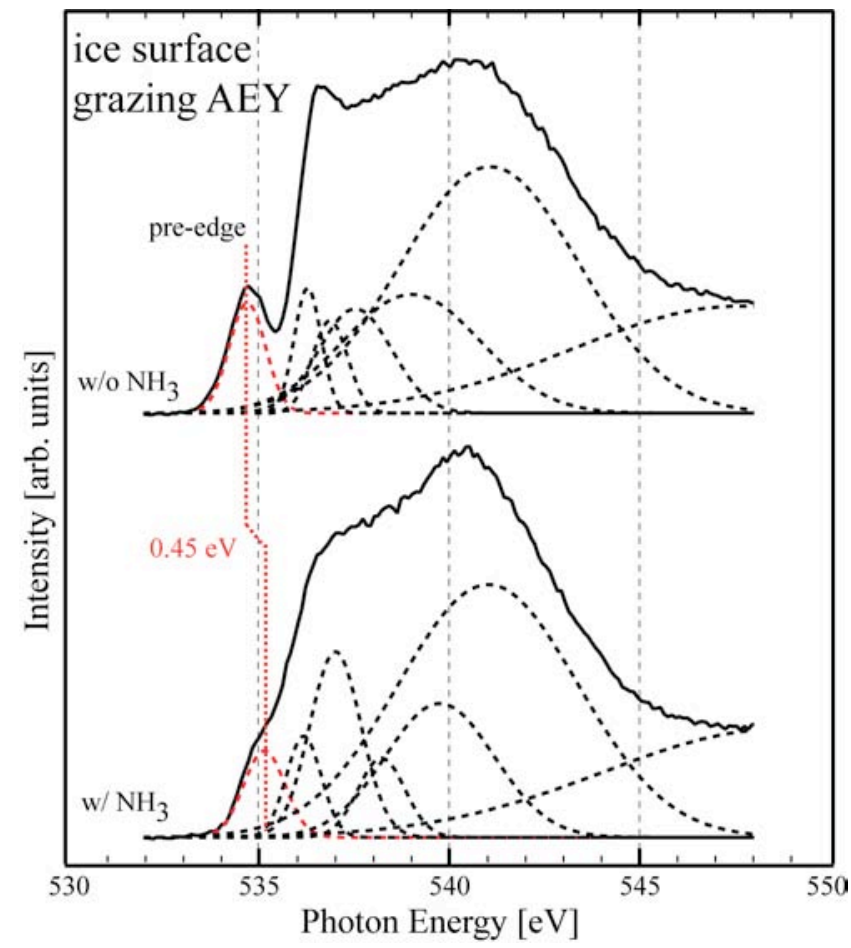

Fig. 27 Surface-sensitive grazing AEY XAS spectra of (a) crystalline ice and (b) $\mathrm{NH}_{3}$-terminated crystalline ice (30). Both spectra are area-normalized and fitted with Gaussian peaks (dashed lines). The red dashed peak in each spectrum indicates the fitted pre-edge peak, which is shifted by $0.448 \mathrm{eV}$ as the surface $\mathrm{O}-\mathrm{H}$ groups are saturated with ammonia. 
In fig. 28 we show a curve-fitting of the XAS spectra from the crystalline ice films with and without ammonia measured with normal AEY which is more bulk-sensitive. The top figure also shows the liquid water spectrum for comparison. The right part of the figure shows a close-up of the pre-edge region. We can fit the crystalline XAS spectrum with two peaks in the pre-edge region, one that has exactly the same energy position as in the surface-sensitive spectrum while the other is a pre-edge component at $0.4 \mathrm{eV}$ higher energy connected to the bulk of ice. Upon ammonia adsorption only the surface pre-edge peak shifts with the same amount as in the surface-sensitive spectrum, whereas the bulk pre-edge peak is at constant energy. In the latter case the bulk peak can be clearly seen. The intensity of the two peaks remains constant. The shift to higher energy is consistent with the difference between TEY and total ion yield (TIY) of crystalline ice in the study of Parent et al. (31). The TIY is based on detection of protons emitted at the surface and is therefore extremely surface sensitive $(31 ; 108 ; 109)$. Note that the number of Gaussians used to fit the spectra in figs. 27 and 28 is arbitrary and that the Gaussians don't reflect discrete transitions. We aimed here at determining the pre-edge position and in order to do this reliably we had to fit the whole spectrum.
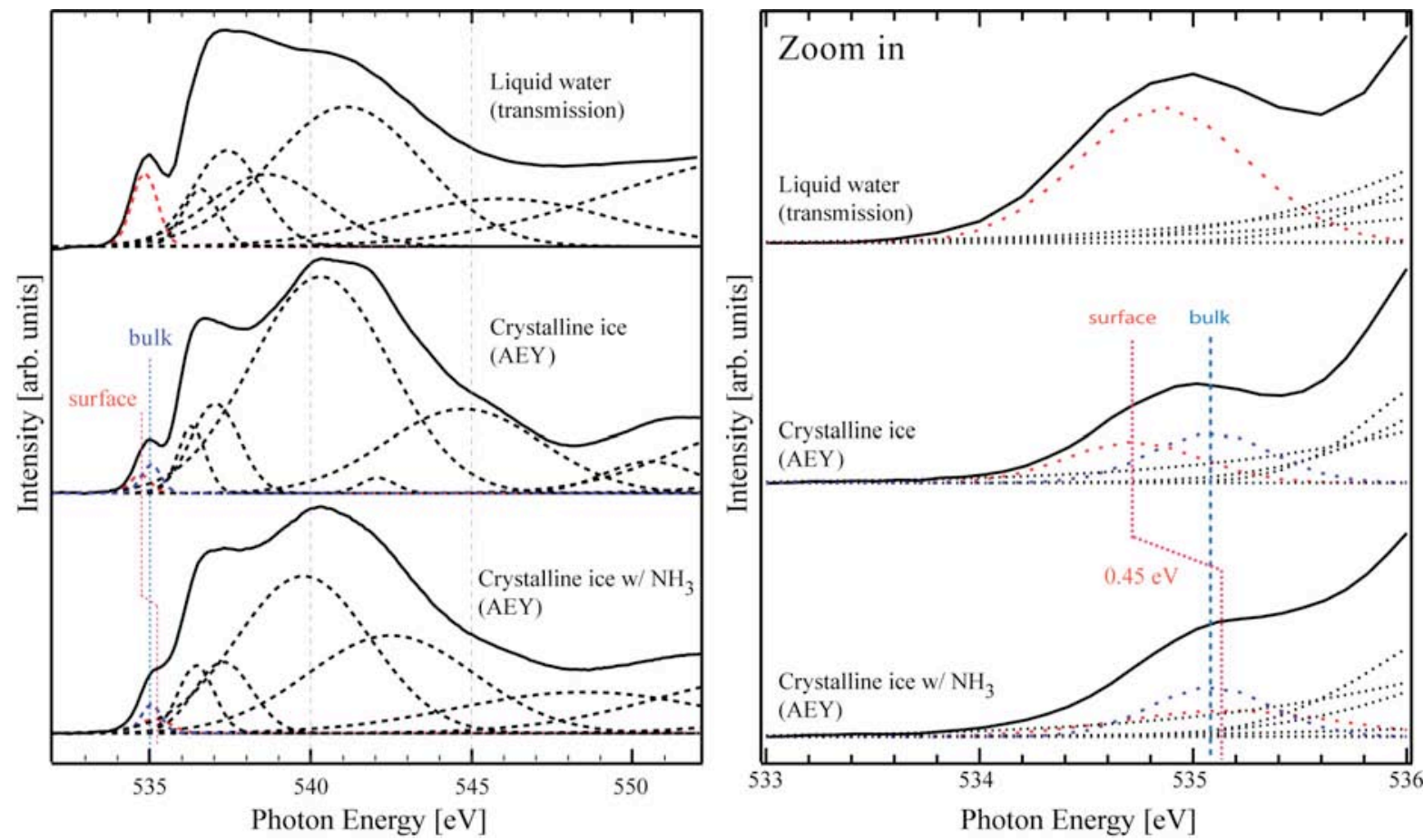

Fig. 28 XAS spectra of (a) room temperature liquid water (transmission mode), (b) 45 layers crystalline ice (AEY), and (c) $\mathrm{NH}_{3}$-terminated crystalline ice (AEY). All spectra are area-normalized and fitted with Gaussian peaks (dashed lines).

We have thereby established that there is indeed a surface pre-edge feature which is related to the dangling $\mathrm{OH}$ bonds and can be assigned to an SD species. Let us now address the criticism by Smith et al. (23) that the pre-edge peak in the Parent et al. study (31) had much higher intensity in comparison to the rest of the spectrum than was seen in the Wernet et al. and Nordlund et al. studies $(3 ; 30)$. They based their comparison on the TIY spectrum of Parent et al. (31) whereas the spectrum in Wernet et al. (3) was obtained with surface-enhanced AEY. As pointed out already in the paper by Parent et al. (31) the large increase in the TIY cross section for the pre-edge feature is connected to two aspects. Promoting an electron into the pre-edge 
feature, which corresponds to occupying a strongly antibonding orbital, will enhance the dissociation probability and increase the cross section for proton emission. The second aspect is that the $\mathrm{OH}$ dangling group is sticking out from the surface and the protons can be emitted directly along the $\mathrm{OH}$ bond direction without any scattering processes whereas the other resonances in the TIY spectrum could give rise to proton emission processes of other OH groups which are oriented into the substrate, which will increase the probability for neutralization. It has previously been shown by Coulman et al. (108) using angle-resolved proton detection that the proton emission from the dangling $\mathrm{OH}$ group is strongly peaked in the forward direction along the $\mathrm{OH}$ group. This clearly shows that the relative intensities in the TIY spectrum are not quantitative but subject to large variations in the proton emission and detection cross section. It is therefore not appropriate to compare relative intensities between TIY and AEY spectra as done by Smith et al. (23).

It is clear that there is a pre-edge peak connected to the bulk in ice. This feature is also seen in XRS studies of various ices $(26 ; 60)$ and in the transmission spectra in fig. 25 . Tse et al. claimed that the pre-edge feature lacks structural sensitivity since it is a core exciton (26). However, in this section we have demonstrated that the energy position of the pre-edge feature is indeed sensitive to the surrounding environment. We observe the lowest energy for the surface of ice at $534.7 \mathrm{eV}$, the position in bulk ice is then shifted to higher energy at $535.05 \mathrm{eV}$ and the highest position is for the ammonia-terminated surface, which is at $535.15 \mathrm{eV}$. As demonstrated in section III. 6 the pre-edge feature corresponds to an excited state that is excitonic in nature, and we thereby agree with Tse et al. (26) about its nature, but disagree with the statement that there is no structural sensitivity. Indeed if we curve fit the liquid water spectrum and normalize the preedge feature with respect to the high energy continuum, which is atomic and not sensitive to the chemical surroundings (excluding EXAFS oscillations), we find that the liquid water spectrum has a 4 times more intense pre-edge feature than the pre-edge peak that remains in bulk ice after ammonia termination as shown in fig 28. We will in section V.1 demonstrate that the pre-edge peak both increases in intensity and shifts to lower energy with increasing temperature of liquid water. Here we can note that the pre-edge is shifted to lower energy at the surface in comparison to the bulk ice. There seems to not only be an intensity effect of the pre-edge peak, but also a sensitivity to the energy position where larger distortions shift the peak more towards the gas phase value.

The intensity of the post-edge feature is not that strong in the spectra of crystalline ice after ammonia deposition, shown in fig. 26. Furthermore, there is a shoulder at $542 \mathrm{eV}$ in the spectra of crystalline ice. The latter can be attributed to a surface feature which is quenched upon ammonia adsorption and much weaker if not absent in the transmission spectra (fig. 25). The post-edge in fig. 1 is significantly higher than in fig. 25 and this is related to the detection technique. Fig. 29 shows XAS spectra of ice grown on $\mathrm{Pt}(111)$ at $130 \mathrm{~K}$ and detected using secondary electron yield (SEY) where close to zero kinetic energy electrons are detected using an electron spectrometer (30). As discussed in section II.1 this gives an extremely bulk-sensitive spectrum and for thin films of ice it means that the whole film is detected in the spectra. Fig. 29 shows spectra of two films which are 10 layers and 50 layers thick. First we note that the bulksensitive spectra have a much higher post-edge than found in the surface-sensitive spectra shown in fig. 25. There seems to be much more disorder in the surface region as manifested in the AEY measured spectrum (30). Furthermore, we note that there is quite a variation of the relative preedge to post-edge intensity in the spectra of the two ice films in fig. 29. The post-edge is much 
more intense in the 50 layer film. This indicates that there must be significant disorder in the surface region which extends down rather deep into the bulk.

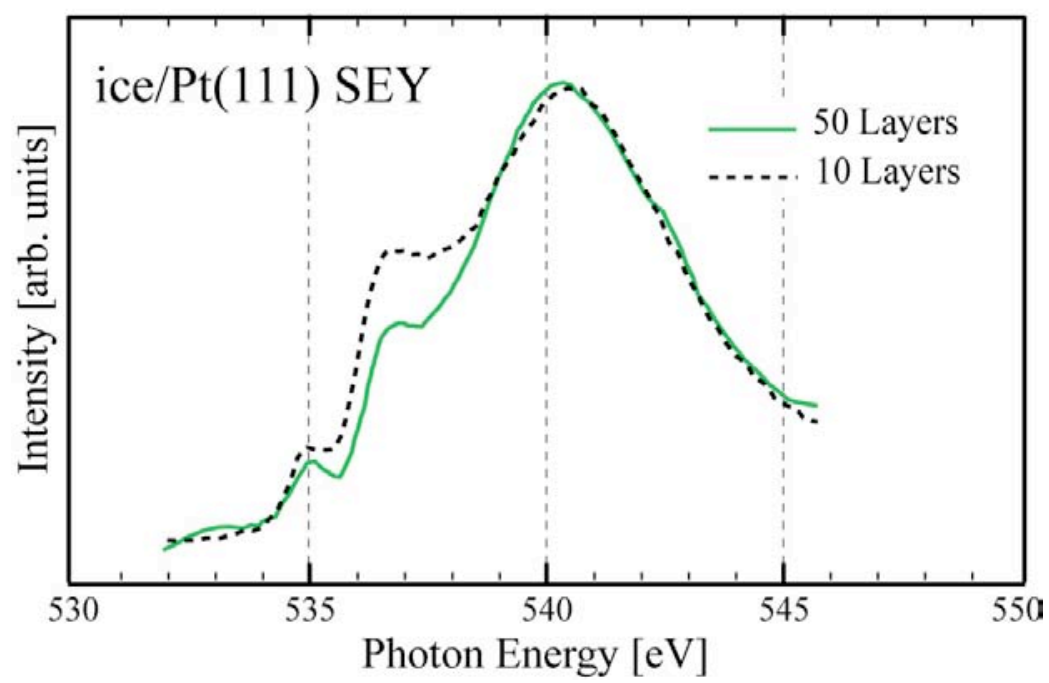

Fig. 29 Two differently prepared ice films on Pt(111) which are 10 (dashed) (30) and 50 (full line) monolayers thick measured with secondary electron yield (SEY).

What is the nature of the pre-edge peak that is also seen in the bulk-sensitive spectra? First we note that the pre-edge feature in amorphous ice has higher intensity than in crystalline ice. It has been seen from a recent Extended X-ray Absorption Fine Structure (EXAFS) study of ice films grown on $\mathrm{Au}(111)$ under UHV conditions that the films do not correspond to a pure phase (104). In Zubavichus et al. (104) the ice films were prepared at 90 and $150 \mathrm{~K}$ and gave XAS spectra with AEY similar to the amorphous and crystalline ice films on $\operatorname{Pt}(111)$ as shown in fig. 25. We thereby expect that the films are rather similar in structure. The EXAFS data of the crystalline phase ( $150 \mathrm{~K}$ preparation) could not be fitted to a structural model of cubic ice $\mathrm{I}_{\mathrm{c}}$ and instead only gave a satisfactory fit when the structural model also included mixtures of highdensity ice structures such as ice II, ice III and high-density amorphous ice (HDA) (104). The amount of the high density structures was higher in the $90 \mathrm{~K}$ prepared ice film than for the $150 \mathrm{~K}$ preparation. The most surprising result was that the $\mathrm{I}_{\mathrm{c}}$ contribution was in minority. This implies that what is often denoted as crystalline ice grown on metal substrates under UHV conditions at temperatures close to desorption is not completely crystalline in nature and most likely contains a mixture of different amorphous structures. Maybe the pre-edge and also the sharp main-edge features can be related to high-density ice structures. The difference between the spectra of lowdensity amorphous ice (LDA) and HDA in the Tse et al. (26) study using XRS is an increase in the pre-edge and main-edge and decrease of the post-edge for HDA.

It was shown earlier by Narten et al. and Jenniskens et al. using x-ray and electron diffraction that condensing water on a hydrophobic substrate at temperatures around $10 \mathrm{~K}$ results in HDA type of ice films being generated $(110 ; 111)$. Heating then causes various transformations between different amorphous phases to take place. We have observed large differences in ice spectra, as shown in fig. 30, when deposited as a few monolayers on $\mathrm{BaF}_{2}$ at two temperatures, $100 \mathrm{~K}$ and $150 \mathrm{~K}$ (112). The low-temperature deposition, where the ice spectrum shows an increased pre-edge and a very intense and sharp main-edge, might generate structures similar to what Narten et al. and Jenniskens et al. reported. This would be consistent with the XRS spectrum of HDA from the Tse et al. study (26) but with much sharper spectral 
features. The ice film on $\mathrm{BaF}_{2}$ deposited at $150 \mathrm{~K}$ shows a more intense and sharper post-edge than for the ice film prepared at the same temperature on $\operatorname{Pt}(111)$. This could indicate a higher fraction of ice $\mathrm{I}_{\mathrm{c}}$ in the film prepared on $\mathrm{BaF}_{2}$. The spectra of the two ice films on $\mathrm{BaF}_{2}$ could perhaps be described as a linear combination of spectra from cubic $I_{c}$ and HDA ice with surface contributions as well. We would anticipate that for a perfect $I_{c}$ crystal the pre-edge and mainedge features have much lower intensity and that the post-edge is more intense and sharper than for the crystalline ice spectra in fig. 25. Furthermore, this would also explain the differences in spectra using SEY techniques with varying film thicknesses shown in fig. 29. This would be consistent with the spectra for ice monolayer structures shown in the next sections where the Evector is parallel to the surface and all waters have saturated all donor H-bonds. The, in that case, only faint remaining intensity of the pre-edge in the water overlayer spectra could be related to asymmetrical $\mathrm{OH}$ vibrations giving intensity in the $4 \mathrm{a}_{1}$ spectral feature in a similar manner as seen in the gas phase spectrum of methane as discussed in section III.5.

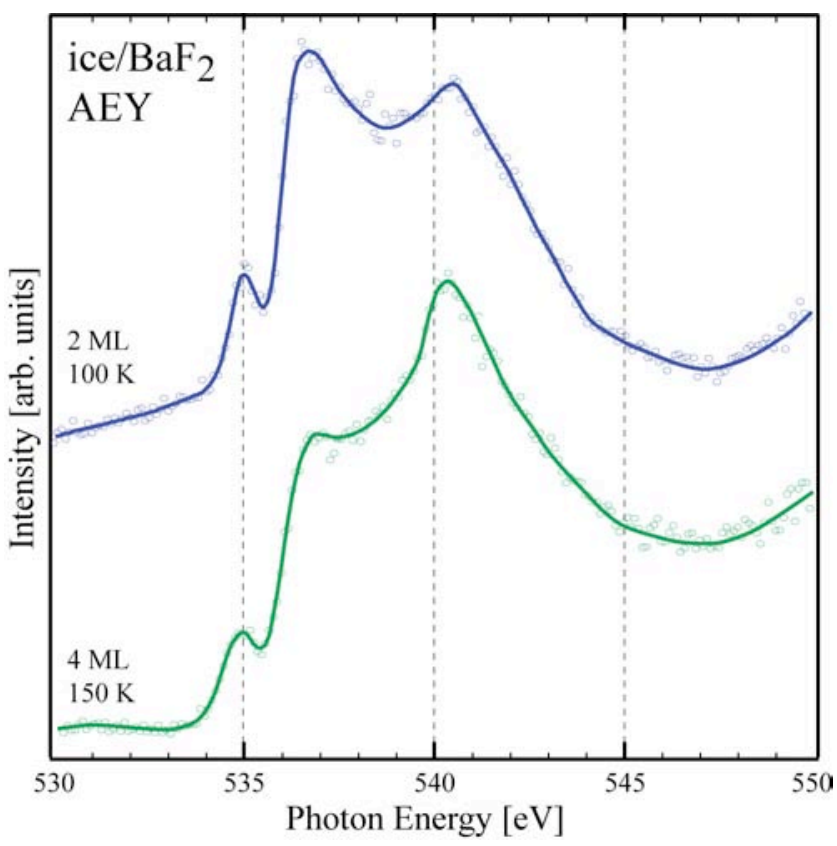

Fig. 30 In-plane polarized XAS spectra of thin ice layers on $\mathrm{BaF}_{2}$ prepared at $100 \mathrm{~K}$ (top) and $150 \mathrm{~K}$ (bottom).

Would the above picture of the spectra of thin ice films be consistent with the Tse et al. study of various bulk ices (26), including high-pressure ones, in which all spectra showed a preedge feature with more intensity than what was discussed above for a hypothetical perfect $I_{c}$ spectrum? The transmission spectra of crystalline and amorphous ice shown in fig. 25 were obtained from $300-500 \mathrm{~nm}$ thin films, which are still thick enough to be regarded as bulk; and the resulting spectra are also rather similar to the XRS spectra by Tse et al. It has been shown with diffraction experiments that there exists a multitude of different states between HDA and LDA (113-116). There seems to be a heterogeneity in the amorphous ices depending on the preparation conditions although LDA has been shown to be homogeneous disordered when prepared from HDA (113). However, maybe there could still be some small but significant amounts of HDA in such ices prepared for the XRS measurements $(26 ; 60)$ and for the transmission spectra in fig. 25. In particular grain boundaries could give rise to regions with 
disorder that extend down rather far into the crystallites, as discussed in connection to fig. 29. In these regions we can speculate that heterogeneities could exist and, if the grains are small, this could give rise to a significant intensity. The same could also be the case for the crystalline phases of ice $I_{h}$ and high pressure ices. Such a picture would then make the discussion of the above thin film ices become consistent with the various bulk ices. However, this is more of a speculative nature and it will be essential to perform further experiments on various forms of ice as thin films and also generated as bulk structures. The ideal would be to generate pure singlecrystal forms of ice, but this is less than trivial.

As a final note on the discussion of the ice spectra we like to bring up that in the Wernet et al. study (3) the shown bulk ice spectrum was obtained by subtracting from the SEY spectrum of 10 layer ice (shown in figs. 1 and 29) the more surface sensitive AEY spectrum in order to enhance the bulk contribution. This generated spectrum has much smaller pre-edge and more intense post-edge than seen in the spectra of crystalline ice from figs. 25 and 29. The assumption that the pre-edge feature is entirely due to the surface of ice is clearly incorrect based on the experimental spectra. However, a perfect ice $\mathrm{I}_{c}$ phase without any disorder or heterogeneity might be close to the spectrum shown as bulk ice in Wernet et al.(3).

\section{IV.2 Two-dimensional overlayers on metal surfaces.}

Here we will discuss another class of model systems for understanding the XAS spectra where we make use of two-dimensional overlayers of adsorbed water on metal surfaces. Due to the directionality of the H-bond and the fact that X-rays produced as synchrotron radiation can be linearly polarized, it is particularly advantageous to study the H-bond environment in lower dimensions where the $\mathrm{x}$-ray E-vector can be aligned to selectively probe the in-plane H-bond interactions. The changes in the electronic structure due to the chemical bonding to the substrate are mainly observed in the out-of-plane orientation of the E-vector (117).

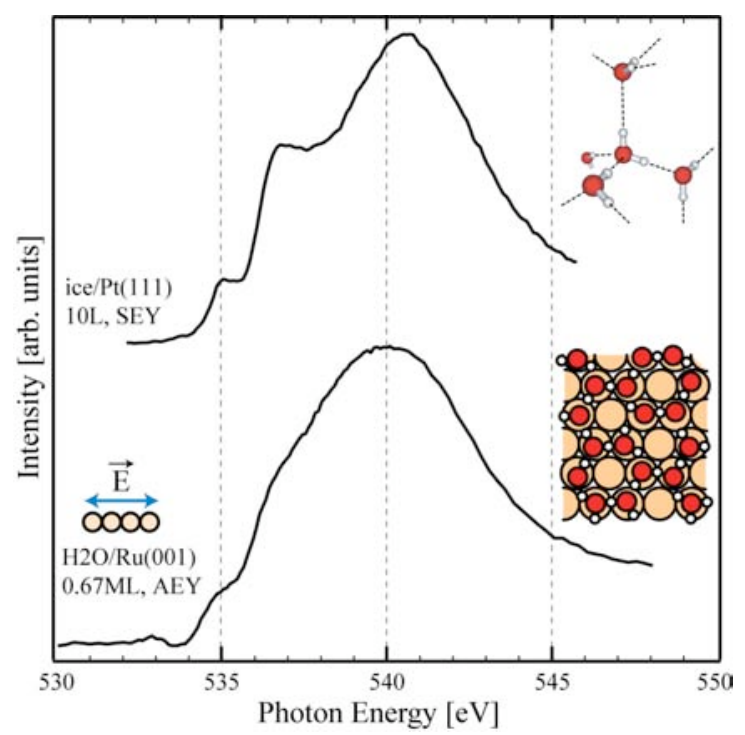

Fig. 31. XAS spectra (top) detected with secondary electron yield (SEY) of 10 ML ice grown on $\mathrm{Pt}(111)$ at $130 \mathrm{~K}$ (30) and (bottom) detected with AEY of a saturated layer of adsorbed water on $\mathrm{Ru}(0001)$ which has been annealed to $150 \mathrm{~K}$.

Fig. 31 shows the in-plane XAS spectrum of a saturated layer of water on $\mathrm{Ru}(0001)$ with a coverage of 0.67 with respect to the surface $\mathrm{Ru}$ atoms (118). The spectrum shows a broad and 
strong post-edge peak. Since the overlayer is at maximum coverage all water molecules have all donating in-plane H-bonds fully saturated $(119 ; 120)$ resulting in nearly negligible intensity in the pre-edge peak. The spectrum is rather similar to the SEY ice spectrum on Pt(111) discussed in the previous section, except for the width of the post-edge and the missing sharp pre-edge. The broad post-edge can be related to strong disorder in the overlayer (120-122). The remaining faint pre-edge feature can be related to vibrational motions that break the symmetry as discussed in the previous section.

Another adsorbate system where the water molecules have all in-plane $\mathrm{OH}$ groups involved in donating $\mathrm{H}$-bonds is the coadsorbate system of $\mathrm{OH}-\mathrm{H}_{2} \mathrm{O}$ on $\mathrm{Pt}(111)$ (120). This overlayer is formed when water reacts with adsorbed oxygen to form $\mathrm{OH}$ groups. The mixed overlayer forms a $3 \times 3$ structure with alternating $\mathrm{OH}$ and $\mathrm{H}_{2} \mathrm{O}$ with equal proportions as shown in fig. 32 (120). The in-plane XAS spectrum in fig. 32a shows a strong post-edge with no intensity in the mainor pre-edge regions, but a tail towards $531 \mathrm{eV}$ which is the onset for the $\mathrm{OH}$ species spectrum contributions (123). This again demonstrates that with both $\mathrm{OH}$ groups in a water molecule involved in H-bonds a strong post-edge appears in the XAS spectrum. If we lower the oxygen coverage prior to the reaction with water we will lower the $\mathrm{OH}$ concentration which will then give rise to defects in the overlayer where water molecules at these sites will not have fully saturated H-bonds (124). Fig. $32 \mathrm{~b}$ and c show XAS spectra for decreasing oxygen coverage and now a growing sharp pre-edge feature becomes visible. This demonstrates again the connection of the pre-edge with weak or broken H-bonds.
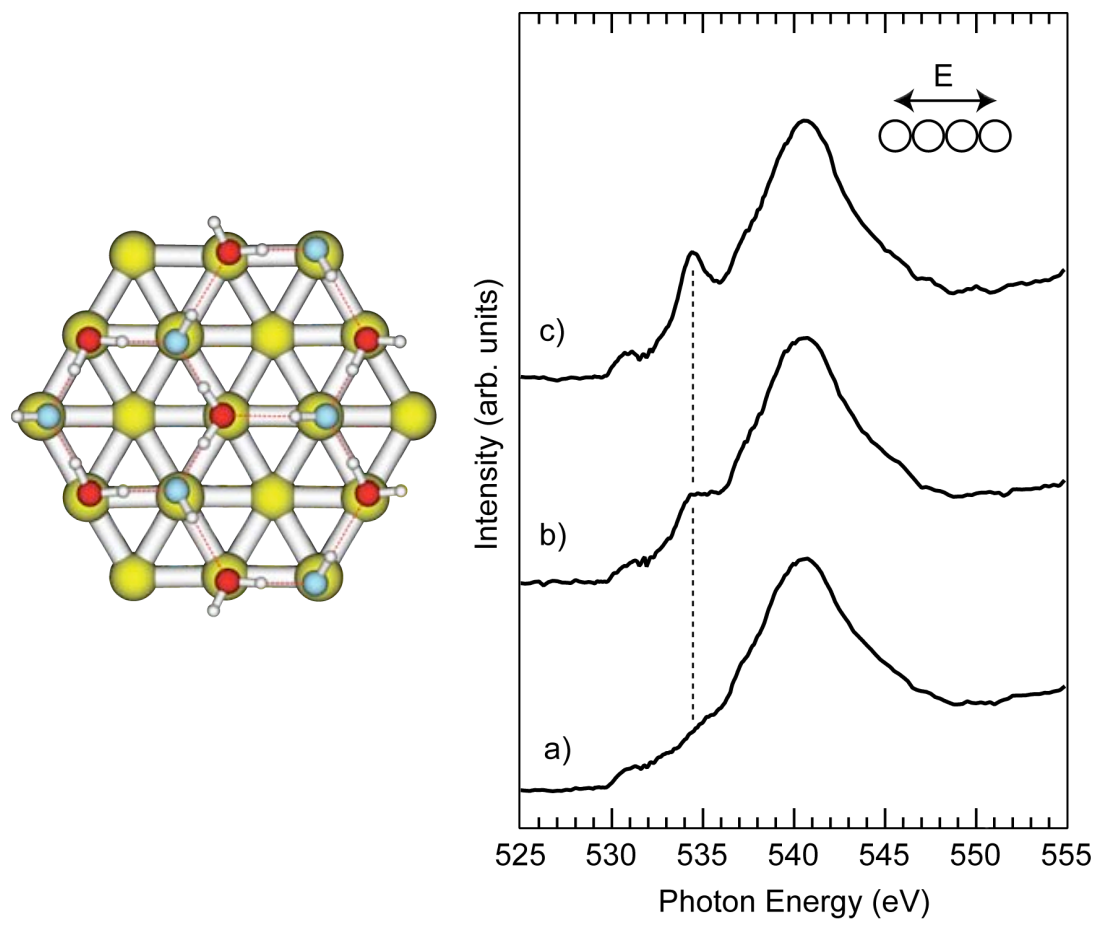

Fig. 32. (left) Structural model of the coadsorbed $\mathrm{OH}-\mathrm{H}_{2} \mathrm{O}$ layer on $\mathrm{Pt}(111)$ (123) and (right) XAS spectra of the overlayer with different initial oxygen coverages.

In these water overlayer structures the orientation of the water molecules parallel to the surface is not well defined such that free and H-bonded OH-groups cannot be separately measured. It would, however, be most interesting if we could utilize the E-vector orientation to 
selectively excite into the molecular states that are made up of $p$-orbitals either along the H-bond or the weak or broken H-bonded group in an SD species.

In fig. 33 we show symmetry-resolved XAS spectra of the amino group in deprotonated glycine (glycinate) on $\mathrm{Cu}(110)$ taken from $(125 ; 126)$. The $\mathrm{Cu}(110)$ surface has two-fold symmetry with the surface $\mathrm{Cu}$ atoms oriented into rows. The glycine molecular skeleton bridges over two $\mathrm{Cu}$ rows on the surface in order to bond through both the carboxylic and amino functional groups $(125 ; 127 ; 128)$. The formation of $\mathrm{H}$-bonds between the carboxylic and amino functional groups on neighboring molecules gives rise to an ordered $(3 \times 2) p g$ overlayer on the $\mathrm{Cu}(110)$ surface with one of the amino hydrogens $\mathrm{H}$-bonded and the other not. This leads to an asymmetrical H-bonding situation in the $\mathrm{NH}_{2}$ group with one strong donating bond whereas the other hydrogen atom is not involved in any H-bonds as shown in fig. 33. Here, the alignment of the E-vector relative to the rows in the open $\mathrm{Cu}(110)$ surface reduces the dimensionality to one, and we can get a very direct connection between geometrical symmetry and x-ray absorption transition amplitudes by selectively orienting the E-vector along either of the two N-H groups. The highly oriented system means that we have three directions defined for the $p$-components, $p_{x}, p_{y}$ and $p_{z}$, respectively. Fig. 33 directly demonstrates the excited state localization on the free $\mathrm{N}-\mathrm{H}$ group due to lowering of the symmetry resulting from the asymmetrical H-bonding. Similar to asymmetrically $\mathrm{H}$-bonded water we observe pre-edge and main-edge features in the spectrum with the E-vector along the free N-H while the post-edge feature is observed with the E-vector along the $\mathrm{H}$-bonded $\mathrm{N}-\mathrm{H}$.

\section{Glycinate on $\mathrm{Cu}(110)$}
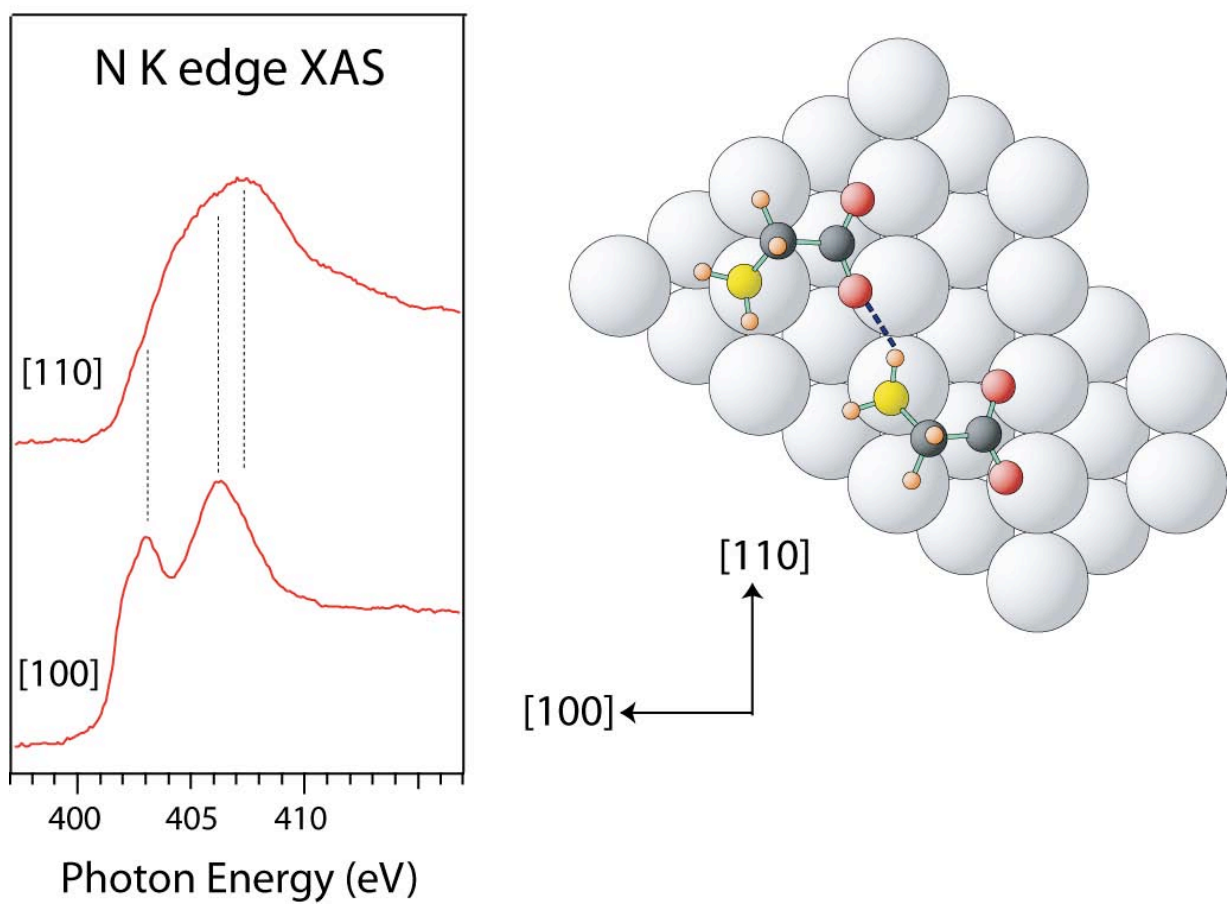

Fig. 33 (left) N K edge XAS spectra of glycinate on $\mathrm{Cu}(110)$ with the E-vector in-plane and either in the 100 or the 110 direction (125). The pre-edge and main-edge spectral features are observed at 403 and $406 \mathrm{eV}$, respectively, in the spectrum with the E-vector aligned with the 100 direction whereas the post-edge is observed at $407 \mathrm{eV}$ in the spectrum in the 110 direction. (right) Structural model of the adsorption geometry of glycinate on $\mathrm{Cu}(110) \mathrm{where}$ the amino group in the lower molecule has two $\mathrm{N}-\mathrm{H}$ where one donates an $\mathrm{H}$-bond to the carboxylate group on a neighboring molecule and the other is non-H-bonded. 


\section{Water and Aqueous Solutions}

In the following we will discuss the current understanding of water that recently has been obtained based on a multitude of new experimental methods. We will base the discussion on new high-resolution temperature-dependent XRS measurements and relate the spectral changes to the previous discussion to make a consistent picture. At the end we will also include the spectral changes as we increase the concentration of simple $\mathrm{NaCl}$ salt solutions.

\section{1 Temperature-dependent XRS spectra}

In Wernet et al. the XRS temperature-dependent spectra, shown in fig. 2, were discussed in terms of an interconversion between SD and DD species (3). Since then two other important experimental findings, using respectively XES and small angle x-ray scattering (SAXS), have been reported supporting this interpretation. First, the XES spectra of water show a split into two peaks in the lone pair $1 b_{1}$ region $(13 ; 25 ; 37)$ denoted in ref. $(25)$ as $1 b_{1}{ }^{\prime}$ and $1 b_{1}{ }^{\prime \prime}$, where the former is close in energy position to the $1 b_{1}$ in crystalline ice and the latter to $1 b_{1}$ in water vapor (25). The two peaks were thereby related to tetrahedral, or DD, species $\left(1 b_{1}{ }^{\prime}\right)$ and H-bond distorted or SD (1 $\left.b_{1} "\right)$ local structures (25) with a relative population of around 1:2 to 1:4 depending on the assumptions in the curve-fitting procedure $(25 ; 38)$. The relative populations based on the XES data are thus within the error bars in the Wernet et al. study (3). Resonantly excited XES spectra at various energy positions in the XAS spectrum make a connection between the two X-ray spectroscopies (25). It was shown that the high energy $1 b_{1}$ " XES peak is linked to the pre-edge spectral feature and the low energy $1 b_{1}{ }^{\prime}$ to the post-edge in XAS $(25 ; 38)$. Since the post-edge feature is clearly enhanced in ice $(3 ; 30)$ it is consistent with that the $1 b_{1}{ }^{\prime}$ is tetrahedral-like, whereas the pre-edge peak, in XES associated to the $1 b_{1}{ }^{\prime \prime}$, has been discussed in terms of distorted H-bonding configurations $(3 ; 21 ; 23)$. The temperature-dependence of both XAS and XES give the same experimental observations where, in XRS, the post-edge diminishes and the pre-edge increases with increasing temperature exactly in the same manner as the $1 b_{1}{ }^{\prime}$ converts to $1 b_{1} "$ in XES $(25 ; 38)$.

Small angle X-ray scattering (SAXS) shows a small enhancement in the low momentum $(q)$ transfer region demonstrating the existence of a density heterogeneity in the liquid (38). The enhancement increases with decreasing temperature which makes a nice link to the increasing anomalies of water with decreasing temperature. The SAXS data at $q=0$ can, via a thermodynamic relationship (129), be related to the isothermal compressibility where a nearly perfect agreement with previous experiments using other techniques is obtained (38). This demonstrates that the enhancement in SAXS is related to density fluctuations in the liquid which increase at lower temperature, but where the time scale of the fluctuations is not yet been determined.

Through the combination of SAXS and x-ray spectroscopy a picture of ambient water as a fluctuating liquid with a bimodal distribution of local structures in terms of spatially segregated strongly tetrahedral patches and H-bond distorted structures is obtained (38). This is also within the range of possible structures that x-ray and neutron diffraction data allow, as shown in a recent analysis using reverse Monte Carlo modeling (39). Since no intermediate structures are seen in the XES or XRS data with increasing temperature and since the scattering process takes place on an attosecond time-scale, much shorter than any time-scale related to H-bond dynamics (73), the two structures can be regarded as static on the time-scale of the experiment and the SAXS data was consequently analyzed in terms of a Guinier analysis developed for static 
macroparticle scattering (38). The derived size of the tetrahedral patches gave a diameter in the order of around $1 \mathrm{~nm}$ and weakly increasing with decreasing temperature (38).

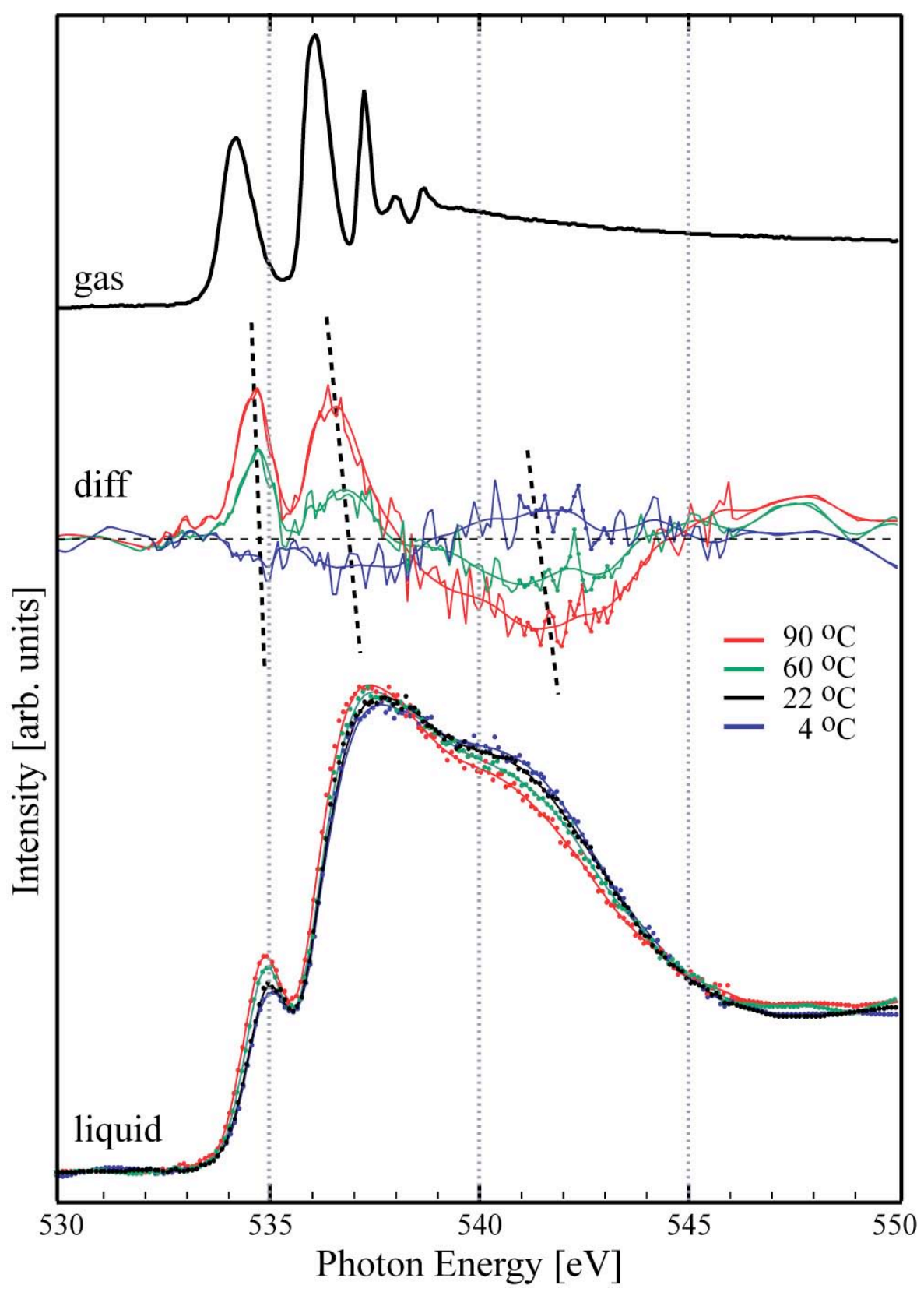

Fig. 34. (bottom) $\mathrm{O}$ K-edge XRS spectra for liquid water $\mathrm{H}_{2} \mathrm{O}$ at various temperatures $\left(4,22,60\right.$, and $\left.90{ }^{\circ} \mathrm{C}\right)(38)$. (middle) Difference spectra relative to the room temperature spectrum $\left(22{ }^{\circ} \mathrm{C}\right)$. Help lines are used to illustrate the shift of the pre-edge and main-edge towards lower energies. (top) The FY-XAS spectrum of gas-phase $\mathrm{H}_{2} \mathrm{O}$ is shown for comparison. All spectra are on a common energy scale where the $3 p$ Rydberg state of the gas-phase spectrum is calibrated to $537.25 \mathrm{eV}$.

Let us now take a look at the XRS high resolution spectra of water at temperatures of 4, 22, 60 and $90{ }^{\circ} \mathrm{C}$ shown in fig. 34 (38). We observe the same trend as shown in fig. 2 and discussed in Wernet et al. (3) with an increase in pre-edge and main-edge and decrease in post-edge intensities. However, there is some additional information that becomes accessible due to the 
improved energy resolution. First we note that there is no additional broadening of the pre-edge peak with increasing temperature. This implies that the structure that gives rise to the pre-edge feature is still relatively well defined although disorder due to temperature must be present. The top part of fig. 34 shows difference spectra with respect to room temperature. We observe an energy shift and enhanced intensity of both the pre-edge and the main-edge features with increasing temperature in the difference spectra. This implies that both the pre-edge and mainedge spectral features shift to lower energies with temperature towards the gas phase values of the $4 a_{1}$ and $2 b_{2}$ spectral peaks. The species generating the distorted H-bonded structures thus become more and more loosely $\mathrm{H}$-bonded with temperature.

Fig. 35 shows the temperature difference between $4-22{ }^{\circ} \mathrm{C}, 22-60{ }^{\circ} \mathrm{C}$ and $60-90{ }^{\circ} \mathrm{C}$ but in this case the intensity scale has been renormalized to the difference in temperature. We can see that the change in the pre-edge and post-edge features is larger at the higher temperatures. This is consistent with the observation in Huang et al. that the change in the intensities of the two XES $1 b_{1}$ peaks is larger for higher temperatures (38). In a Boltzmann type of analysis, assuming that entropy changes can be neglected, it was shown in Huang et al. that the energy difference between tetrahedral and distorted H-bonded structures increases with increasing temperature (38). Furthermore, that in the XRS spectrum the pre-edge and main-edge features both shift in energy towards gas phase with increasing temperature is also made consistent in Huang et al. with the shift of the distorted H-bond $1 b_{1}$ " XES peak towards the gas phase position (38). This directly implies that the H-bond distorted species becomes more thermally excited and changes its structure becoming more disordered with increasing temperature. Although the tetrahedral XES $1 b_{1}$ peak remains at constant energy position with temperature (38) we observe a change in the XRS difference spectra in the post-edge region. Fig. 35 shows that both the width increases and the center energy position shifts to higher energy for the higher temperature difference due to loss of intensity at higher energy. Following the concept of "bond length with a ruler", discussed in section III.7, we would interpret this as preferential loss of tetrahedral structures with shorter H-bond distances at higher temperatures. This indicates that the tetrahedral structures undergoes some expansion with increasing temperature which is, however, too small to cause any detectable shift in the XES $1 b_{1}{ }^{\prime}$ peak position. We also see in fig. 35 that the mainedge change is different in the different temperature intervals becoming sharper with increasing temperature.

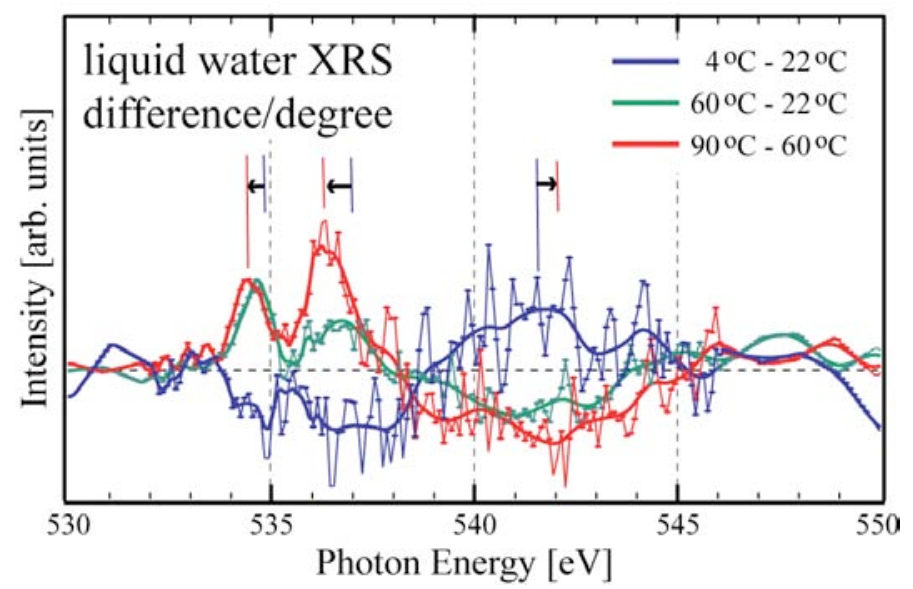

Fig. 35. O K-edge XRS difference taken at various temperature ranges. The difference spectra have been normalized to the temperature difference to show the temperature effect on the XRS spectra per degree. 
The picture that emerges is that the tetrahedral-like structure forms as a low-energy/lowentropy structure of lower density than the average of the liquid (38). The higher density, thermally excited $\mathrm{H}$-bond distorted structure, on the other hand, is a high entropy structure where the higher density reduces the loss of enthalpy through a larger number of, but less specific, Hbond interactions. As we increase the temperature the fraction of tetrahedral structures is decreased while those present at the higher temperature undergo only minor changes whereas the H-bond distorted become more thermally excited with a concomitant more significant expansion; the availability of thermally excitable degrees of freedom allows this species to contribute more to the entropy.

Let us connect to the discussion in section IV.1 regarding the spectra of various ices. It is clear that the overall spectrum of liquid water is rather remote from that of cubic ice, in particular considering that the experimental ice spectrum also contains contributions from other forms of ice. However, the spectrum of the HDA type of ice has a close resemblance to the water spectrum. Fig. 36 shows a comparison of the XRS spectrum of water at $22{ }^{\circ} \mathrm{C}$ and the XRS spectrum of HDA as obtained by Tse et al. (26). We note a clear similarity with a high intensity in both the pre-edge and main-edge spectral regions. At the bottom of fig. 36 we also show the XAS spectrum of a thin amorphous ice film on $\mathrm{BaF}_{2}$ deposited at $100 \mathrm{~K}$ (also shown in fig. 30). This ice film was speculated in section IV.1 as being similar to HDA. There is a strong and sharp pre-edge and main-edge feature but also clearly a discernible post-edge. Somehow it looks similar to the water spectrum, but with the difference that all spectral features are much sharper.

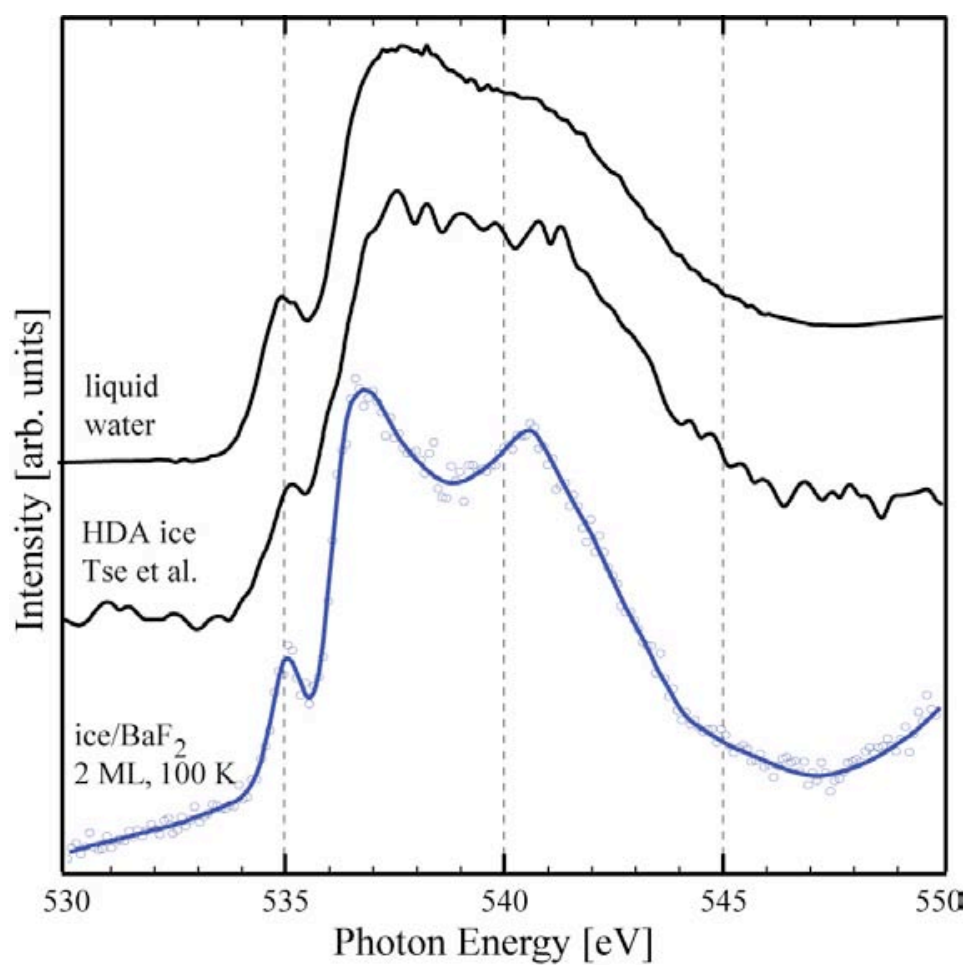

Fig. 36. O K-edge XRS spectra comparing liquid water at room temperature with high density amorphous (HDA) ice (26) and ice on $\mathrm{BaF}_{2}$ from fig. 30. The energy scale is calibrated using the XRS spectrum of liquid water $\mathrm{H}_{2} \mathrm{O}$ in (26).

The question is if some of the post-edge is related to tetrahedral structures. In Huang et al. (38) it was put forward that the spectra of the two fluctuating structures in water show 
resemblance to those of the amorphous ices which spectroscopically would indicate a model of water in terms of high density water (HDL) and low density water (LDL) similar to what has been proposed by Stanley in the supercooled water regime (130-134). The distorted H-bonded structures would then correspond to HDL which has some resemblance to HDA but is thermally excited and thereby representing a significantly expanded structure. The existence of the two fluctuating structures in water at ambient conditions would then simply be a consequence of the by Stanley and coworkers proposed $2^{\text {nd }}$ critical point $(133 ; 134)$. The domination of HDL at ambient temperatures is then a reflection of the fact that it is located on the high-temperature side of the phase separation, or Widom line $(135 ; 136)$ (defined as an extension of the coexistence line between low- and high-density liquid in the "one-phase region") whereas the tetrahedral structure is on the low-temperature side.

What can be the structure of the HDL species? We have shown throughout this review that structures, such as the surface of ice, defects in the mixed $\mathrm{OH}-\mathrm{H}_{2} \mathrm{O}$ overlayer on $\mathrm{Pt}(111)$ and $\mathrm{H}$ bonded glycinate on $\mathrm{Cu}(110)$, corresponding to asymmetrically H-bonded situations with one strong donating H-bond and the other weak or broken, give rise to sharp pre-edge and main-edge structures. We know for HDL structures, as obtained with increasing pressure in water, that diffraction indicates a major change from tetrahedral structures (137-139). In the O-O pair correlation functions the $4.5 \AA$ next-nearest neighbor distance is missing and instead a new correlation is enhanced at $3.5 \AA$ (137-139). The latter has also been proposed to exist at ambient conditions in the diffraction data (140-142). The question is if the $3.5 \AA$ can be regarded to be in the first coordination shell and then corresponds to the weak or broken $\mathrm{H}$-bond in an asymmetrical situation. Since we observe an increase in the $3.5 \AA$ and decrease in the $2.75 \AA$ peaks with increasing temperature in the O-O correlation $(139 ; 141)$ we can assume that the conversion of LDL to HDL leads to much more loosely H-bonded structures. Since HDL will be of slightly higher density than the tetrahedral LDL, the HDL with increasing longer distances will require a larger number of molecules in the first coordination shell but with less specific $\mathrm{H}$ bond interactions. The question is if the HDL structures could resemble what has been discussed as SD or asymmetrical species. Although we do not yet know let us for the time being assume that this would be the case. The changes in the XRS spectra in fig. 34 would then indicate, through the shifts of the pre-edge and main-edge energy positions towards the gas phase value, that the SD structures convert more towards a non-donor (ND) type of structure with increasing temperature. If the SD species also contribute to the first peak in the O-O correlation through its strong H-bond we would expect that this peak in the radial distribution function should shift to longer distances if the structures become more ND-like. This is indeed observed in the diffraction data $(139 ; 141)$. Furthermore, we can anticipate that the less specific H-bonds in the HDL component should soften the librational modes resulting in a downward shift in energyspacing allowing these modes to be thermally populated. It was proposed in Leetmaa et al. that the frequency shift in non-linear spectroscopy in the $\mathrm{OH}$ stretch region on ultrafast time scales could be interpreted in terms of the non-H-bonded $\mathrm{OH}$ in a librationally highly excited SD species trading roles with the $\mathrm{H}$-bonded $\mathrm{OH}$ of the same molecule, a process which does not change the H-bonding situation (5). As we increase the temperature the HDL becomes thermally excited, expanding the bond distance which allows for a further softening of the libration modes and the successful attempts where the two $\mathrm{OH}$ are trading roles become more prominent. Eventually there could be no barrier at all and the SD species has become fully an ND species. However, this could appear at very high temperatures maybe close to supercritical water, which does show a large fraction of ND species (64). 


\section{2. Difference between normal and heavy water}

Investigations of the structure and dynamics of light $\left(\mathrm{H}_{2} \mathrm{O}\right)$ and heavy $\left(\mathrm{D}_{2} \mathrm{O}\right)$ liquid water provide an experimental way of addressing isotope quantum effects in the liquid, associated with a number of observed differences in macroscopic properties (143). Notably, several x-ray and neutron scattering experiments have measured the difference in the derived $\mathrm{O}-\mathrm{O}$ pair distribution functions upon $\mathrm{H} / \mathrm{D}$ substitution (144-151) to find that $\mathrm{H}_{2} \mathrm{O}$ at room temperature is similar to $\mathrm{D}_{2} \mathrm{O}$ if the temperature of the latter is raised by $\sim 5^{\circ} \mathrm{C}$.

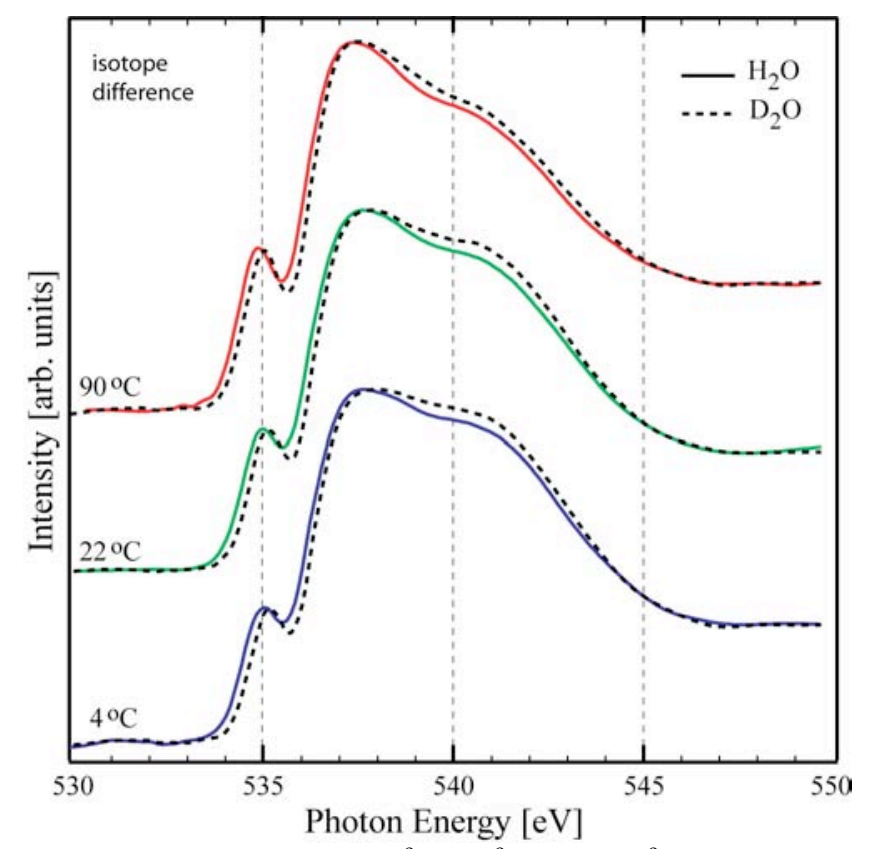

Fig. 37. O K-edge XRS spectra of $\mathrm{D}_{2} \mathrm{O}$ and $\mathrm{H}_{2} \mathrm{O}$ at $4^{\circ} \mathrm{C}, 22^{\circ} \mathrm{C}$, and $90^{\circ} \mathrm{C}$. Due to the small differences, only the splines for the spectra are shown for clarity (in Figure 34 both the raw spectra and their splines are shown).

Despite the small isotope effect, XRS is sensitive to H/D substitution, as was recently shown in Bergmann et al. (59), and the effect can be studied by analyzing the spectral differences in relation to, e.g., temperature effects. In fig. 37, we show an expanded dataset with new high resolution $(0.5 \mathrm{eV}) \mathrm{XRS}$ spectra, where special care has been given to the relative energy scale (error bars within $50 \mathrm{meV}$ ). For each temperature we show the area normalized and smoothened spectra for both $\mathrm{H}_{2} \mathrm{O}$ and $\mathrm{D}_{2} \mathrm{O}$ (see the liquid water data for raw data, fig. 34).

The most obvious difference between $\mathrm{H}_{2} \mathrm{O}$ and $\mathrm{D}_{2} \mathrm{O}$ in the figure (left) is the apparent shift of all $\mathrm{D}_{2} \mathrm{O}$ spectra to higher energy. This can be understood by the difference in ZPE (Zero Point Energy) as schematically illustrated in fig. 38 (left). Although the details of the different final state potential energy surfaces vary with excitation energy, all core-hole states along the XAS profile are dissociative for H-bonded water (152), and can be schematically represented by a steeply sloping potential along a generalized H(D) dissociation coordinate as depicted in figure 38 (left); since the potential is dissociative or only weakly bound there is no zero-point energy difference for the isotopes. In the ground state there is, however, and, since the ZPE is lower for $\mathrm{D}_{2} \mathrm{O}$, we would thus expect the excitation energy for $\mathrm{D}_{2} \mathrm{O}$ to be higher than for $\mathrm{H}_{2} \mathrm{O}$ by the difference in vibrational $\mathrm{ZPE}$ in the ground state. Experimentally, based on the isotope difference in the onset of VUV absorption spectra of $\mathrm{H}_{2} \mathrm{O}$ and $\mathrm{D}_{2} \mathrm{O}(153)$, the vibrational zero-point energy 
of ground-state water is lower in $\mathrm{D}_{2} \mathrm{O}$ than in $\mathrm{H}_{2} \mathrm{O}$ by about $0.16 \mathrm{eV}$. This shift is in analogy with the correction applied in VUV radiation chemistry measurements of liquid water (154) for which the final state potential is also dissociative in the low-energy region. The exact final state potential is of course different from the core-hole excited case in XAS, but the effective shift related to differences in the ground state vibrational energy for vertical transitions should be the same as long as the final state is dissociative. Early ZPE calculations based on perturbation theory calculations of triatomic molecules (155) led to a similar value of about $155 \mathrm{meV}$ (156) for $\triangle \mathrm{ZPE}$, whereas recent $a b$ initio methods applied to a large number of clusters find slightly higher values of $\sim 170-185 \mathrm{meV}$ depending on the local structure. When we correct for the isotope difference in zero point energy, $\triangle \mathrm{ZPE}$, using the experimental value of $160 \mathrm{meV}$ (153), we find that the absolute energy scale for the pre-edge and main edge transitions are very well aligned between $\mathrm{H}_{2} \mathrm{O}$ and $\mathrm{D}_{2} \mathrm{O}$ (fig. 38 b).

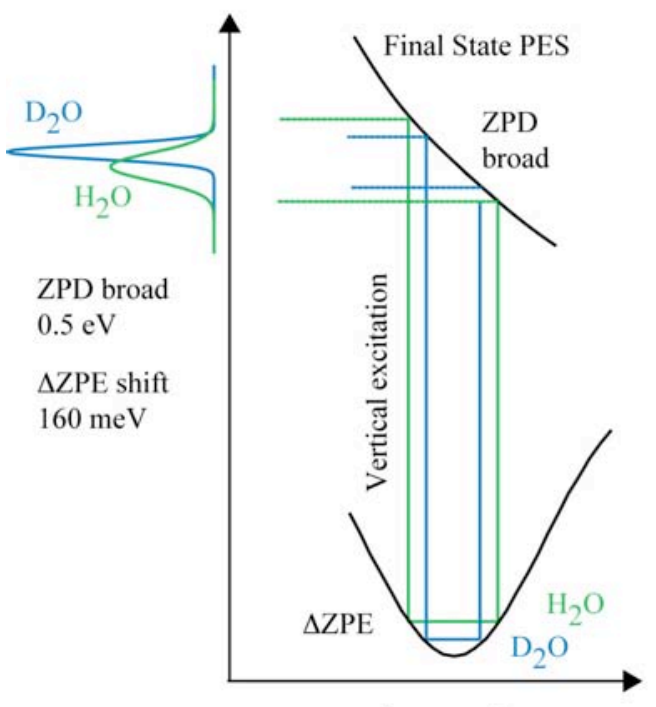

nuclear coord

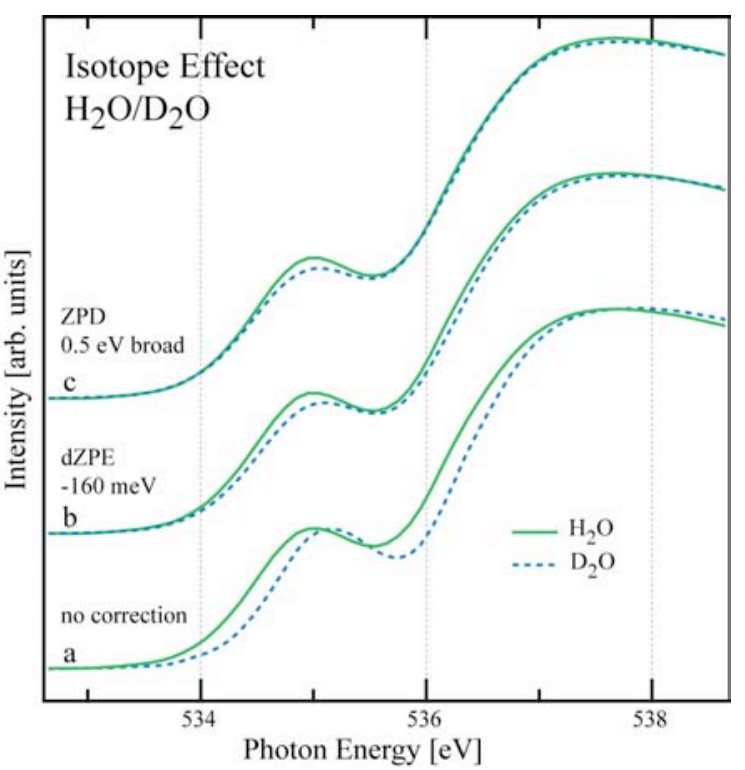

Fig. 38. (left) Schematic drawing illustrating the isotope effect resulting from the Zero Point Distribution, denoted ZPD, and the Zero Point Energy, denoted ZPE. (right) $\mathrm{O}$ K-edge XRS spectra of $\mathrm{D}_{2} \mathrm{O}$ and $\mathrm{H}_{2} \mathrm{O}$ at $22^{\circ} \mathrm{C}$. From bottom to top a) the experimental (splined) XRS spectra b) $\mathrm{D}_{2} \mathrm{O}$ shifted by $+160 \mathrm{meV}$ to compensate for the ZPE. c) $\mathrm{D}_{2} \mathrm{O}$ convoluted with a Gaussian broadening with full width at half maximum of $0.5 \mathrm{eV}$ to compensate for the ZPD.

The ground state zero-point spatial probability distribution (ZPD) also leads to a difference in line-width of the transition. As the schematic shows, a more confined distribution in space for the heavier isotope can be expected to produce a narrower distribution in the vertical transition to a dissociative potential. This is clearly observable in the experimental spectra as a narrower preedge peak in $\mathrm{D}_{2} \mathrm{O}$. We compensate for this zero point effect by convoluting the $\mathrm{D}_{2} \mathrm{O}$ spectrum with a Gaussian function, chosen to have $0.5 \mathrm{eV} \mathrm{FWHM,} \mathrm{similar} \mathrm{to} \mathrm{the} \mathrm{analysis} \mathrm{of} \mathrm{Bergmann} \mathrm{et}$ al. (59). In the $\mathrm{D}_{2} \mathrm{O}$ spectrum corrected for both zero point effects described above (fig. 38c), we find a close resemblance to the $\mathrm{H}_{2} \mathrm{O}$ spectrum with small but significant dissimilarities discussed as follows. In fig. 39 we show the zero point corrected XRS spectra for $\mathrm{H}_{2} \mathrm{O}$ and $\mathrm{D}_{2} \mathrm{O}$ at the three different temperatures (ZPE shift of $160 \mathrm{meV}$ and ZPD broadening of $\mathrm{D}_{2} \mathrm{O}$ with $0.5 \mathrm{eV}$ ). The isotope difference spectra at each temperature are displayed in the top panel. For comparison we show the difference spectrum between $4^{\circ} \mathrm{C}$ and $90^{\circ} \mathrm{C} \mathrm{H}_{2} \mathrm{O}$ divided by 8.6 , representing the 
spectral change per $10^{\circ} \mathrm{C}$. We note that the isotope effect as seen through XRS is rather insensitive to the temperature, and that there are similarities between the differences upon H/D substitution and the changes induced by decreasing the temperature of $\mathrm{H}_{2} \mathrm{O}$ water. However, a structural effect similar to a temperature change, as has been suggested based on other local probes, such as X-ray and neutron diffraction measurements (144-151), can only partly account for the observed differences. For the $\mathrm{H}_{2} \mathrm{O}-\mathrm{D}_{2} \mathrm{O}$ isotope difference spectra at each temperature (top panel) we observe an increase in pre-edge intensity for $\mathrm{H}_{2} \mathrm{O}$ over $\mathrm{D}_{2} \mathrm{O}$, which is not accompanied by a similar increase in the main-edge as would be expected for a temperature effect; the loss in post-edge intensity for $\mathrm{H}_{2} \mathrm{O}$ compared to $\mathrm{D}_{2} \mathrm{O}$ is furthermore mostly at lower energies (lacking the higher energy component around $543 \mathrm{eV}$ seen in the temperature difference spectrum). The interpretation given in Bergmann et al. (59) was that isotope substitution affects the local asymmetry in the H-bonded network: H-bonding configurations are more asymmetric on the donor side for $\mathrm{H}_{2} \mathrm{O}$ than for $\mathrm{D}_{2} \mathrm{O}$ as seen in the difference spectra through enhanced preedge intensity for $\mathrm{H}_{2} \mathrm{O}$ without concomitant main-edge enhancement and through the main difference in the post-edge feature occurring at lower energy than when induced by an increased temperature. The present data reaffirm the experimental results in Bergmann et al. (59). Regarding the interpretation in Bergmann et al., we maintain that - other than the zero point effect - the stronger pre-edge indicates that the species that give rise to the pre-edge and mainedge intensity, i.e. the high-entropy highly disordered species with large H-bond asymmetries, are even more elongated on the weakly bonded donor side in $\mathrm{H}_{2} \mathrm{O}$ relative to $\mathrm{D}_{2} \mathrm{O}$.

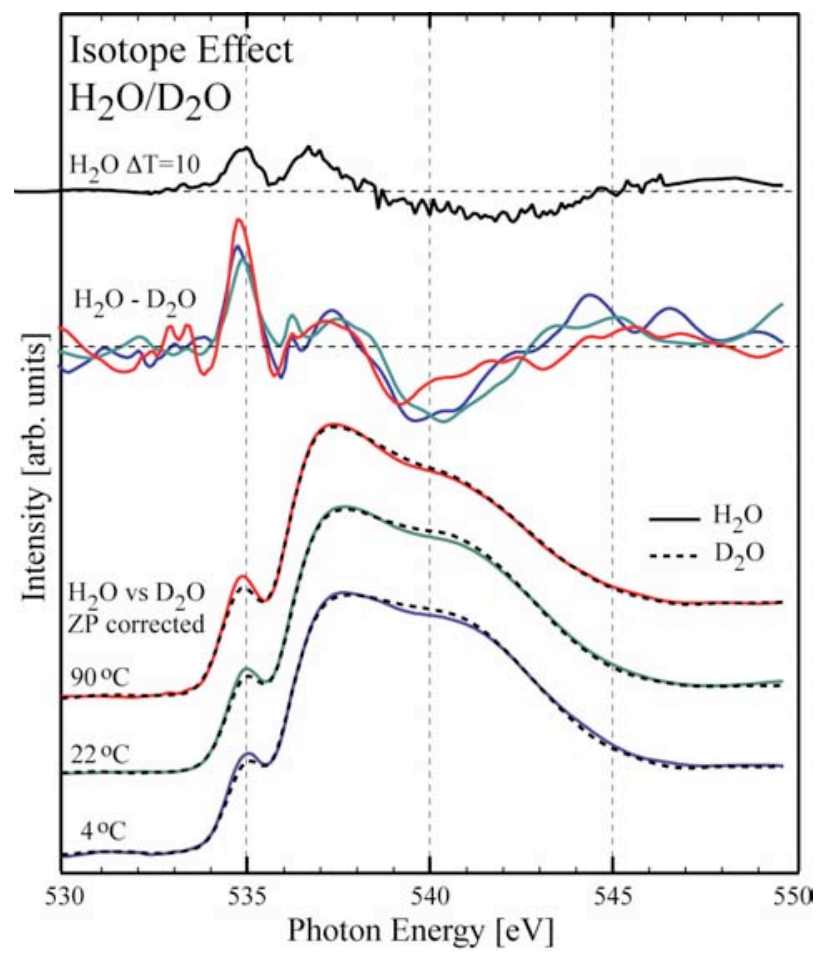

Fig. 39. (bottom) $\mathrm{O}$ K-edge $\mathrm{XRS}$ spectra of $\mathrm{D}_{2} \mathrm{O}$ and $\mathrm{H}_{2} \mathrm{O}$ at $4^{\circ} \mathrm{C}, 22^{\circ} \mathrm{C}$, and $90^{\circ} \mathrm{C}$ after the $\mathrm{D}_{2} \mathrm{O}$ spectra have been shifted by $+160 \mathrm{meV}$ and broadened by $0.5 \mathrm{eV}$ to compensate for zero-point effects.

The differences in the post-edge between the isotopes are found at lower energy than for a temperature increase which we would interpret, following the "bond length with a ruler" 
concept and the discussion in section III.7, as an H-bond shortening, or a preferential loss of structures with longer $\mathrm{H}$-bonds for $\mathrm{H}_{2} \mathrm{O}$ relative to $\mathrm{D}_{2} \mathrm{O}$. The interpretation in Bergmann et al., (59) based on theory, was that this shift indeed indicates an H-bond shortening, and it was argued that the H-bond shortening took place for the same SD species that gave rise to the H-bond elongation on the other donor side. We note that Soper and Bemore (149) have found an $\mathrm{OH}$ internal bond elongation and an associated H-bond lengthening based on analysis of x-ray and neutron diffraction data. This is consistent with shift in the post-edge position and interpretation in the Bergmann et al. It should be noted, however, that the difference in zero-point distribution could also have non-negligible effects on the shape of the post-edge feature resulting in a broader post-edge for the lighter isotope. This should give rise to a more narrow distribution in $\mathrm{D}_{2} \mathrm{O}$ compared to $\mathrm{H}_{2} \mathrm{O}$ as found in theoretical simulations of the water ice spectrum (28); this was not taken into account in the previous study (59). Lacking detailed knowledge of the appropriate broadening to apply to compensate for this additional zero-point-distribution effect, we should at this point partly relax the conclusions based on the post-edge difference.

Regarding the isotope difference as a function of temperature, if anything, there is a sharpening of the pre-edge at higher temperature and an additional shift and slight broadening of the post-edge region (see isotope difference spectrum at $90^{\circ} \mathrm{C}$ ). These are small differences which should not be over-interpreted, but we can speculate about their origin. As discussed in the previous section we could in the interpretation of the HDL structure in terms of SD species anticipate that it becomes more of ND character as the temperature increases and the more thermally excited libration modes make for faster switching between H-bonded and non-bonded $\mathrm{OH}$. There will still be a barrier between the conformations but the lighter isotope could allow for more tunneling. This could potentially increase the pre-edge intensity since the pre-edge is more intense in ND structures than in $\mathrm{SD}(3 ; 6)$.

Overall we observe a shift in the fluctuating equilibrium slightly towards LDL for the heavier isotope similar to a lowering in temperature with a magnitude which is of the order of $10^{\circ} \mathrm{C}$ in close agreement with conclusions based on diffraction data. However, there are some additional spectral changes which are beyond such a simple interpretation. The difference in the pre-edge peak intensity is larger at higher temperatures and there is a shift in the post-edge region of the difference spectra which deviates from the temperature difference. The latter is also enhanced at higher temperature. This could indicate that there are quantum effects which are observed in XRS but are not detectable in diffraction. However, a full understanding of these subtle effects will require a detailed dynamical model of the liquid together with spectral calculations.

\section{3. The effect of ion hydration in $\mathrm{NaCl}$ aqueous solutions}

In nature, water rarely exists in its pure bulk form. In many environmental and biological systems, water acts as a solvent and the most common solutes are ions. Let us now briefly address how ions such as dissolved $\mathrm{NaCl}$ could affect the balance between HDL and LDL in water and how XAS could be used to monitor the resulting structural changes.

The most commonly used description for the effects of ions on the structure of water as either structure-making or structure-breaking is based on macroscopic properties. However, the extent of the water-ion interaction at the molecular level is not yet fully understood. $\mathrm{NaCl}$ solutions have in the past been studied with XAS using both FY and TEY XAS detection schemes $(48 ; 157)$. Fig. 40 shows transmission XAS spectra of pure water and 1-6 molar $\mathrm{NaCl}$ solutions with difference spectra with respect to pure water shown in the top part of the figure. 
We clearly see an increase in the pre-edge and main-edge and decrease of the post-edge features with increasing concentration. The changes in the main-edge and post-edge features seem to vary linearly with concentration whereas the pre-edge shows a larger change between pure water and the $1 \mathrm{~m}$ solution. It is clear that the change in the spectra is mainly seen for high concentrations at $1 \mathrm{~m}$ or higher, which implies that the restructuring of the H-bond network will only be in the proximity of the ions. It was proposed by Cappa et al. (157) that most of the spectral changes are related to modifications of the electronic structure of the water molecules due to the electric field generated by the ions. This was based on spectrum calculations, but there are many questions around how the calculations were carried out (28). In Näslund et al. (48) the spectral changes were discussed in terms of conversion between DD and SD species, which in the current picture would mean LDL and HDL structures. Based on the spectral changes this would imply that for $\mathrm{NaCl}$ solutions we have a conversion of LDL to HDL. We note that the changes in the spectra are rather similar to what was observed with increasing temperature, but with one exception. There are no shifts in the pre-edge or main-edge spectral features as we convert LDL to HDL in $\mathrm{NaCl}$ solutions. If the conversion of LDL to HDL is at constant temperature we could expect that thermal excitations would remain constant. Maybe this would be the change as expected from an increase in pressure at constant temperature where low density is converted into high density. Indeed it has been proposed by Leberman et al. based on neutron diffraction measurements that the change in structure in $\mathrm{NaCl}$ solutions in comparison to pure water is similar to an increase in pressure (158). It has also been proposed, as derived from x-ray diffraction, that the changes in the $\mathrm{O}-\mathrm{O}$ pair correlation function due to increasing pressure and increasing temperature are rather similar (139). There is thereby a consistent argument for the interpretation along the lines of Näslund et al., but in a new terminology in terms of LDL to HDL conversion.

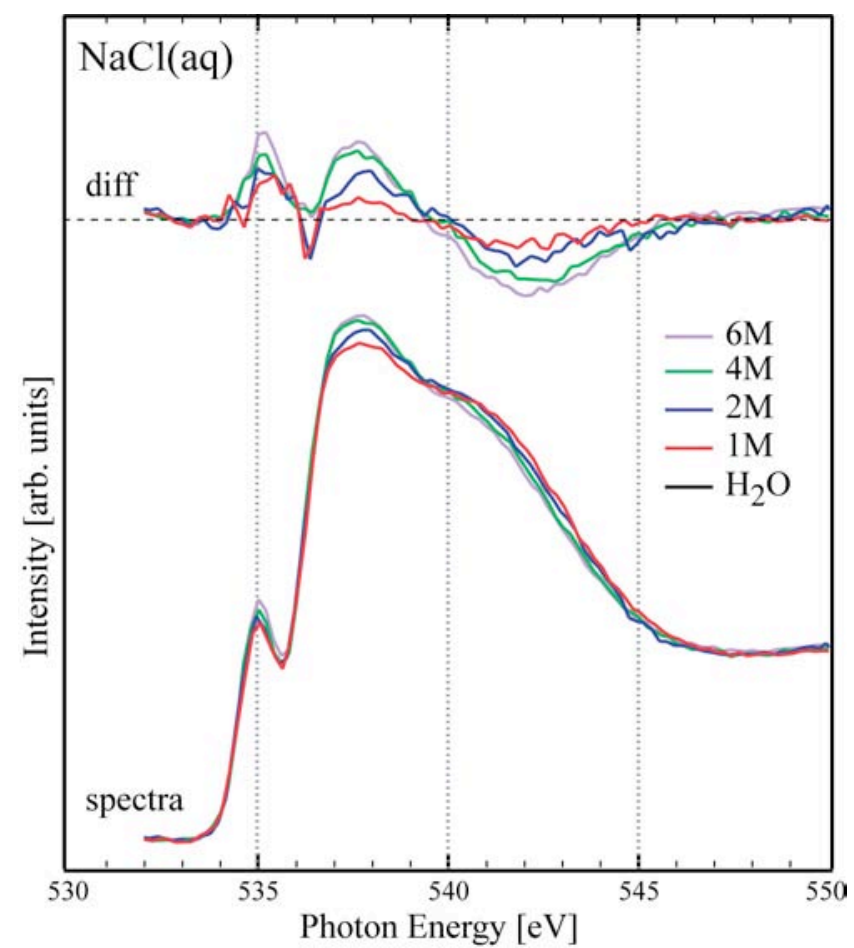

Fig. 40. XAS spectra of $\mathrm{NaCl}$ solutions with increasing concentration measured in transmission mode, compared with the spectrum of pure water. The difference spectra are shown at the top of the figure. 


\section{Summary}

In the previous sections we have focused on the experimental content in XAS/XRS based on various model experiments and on our basic understanding of the x-ray absorption process, demonstrating the general applicability of x-ray absorption spectroscopy to analyze H-bonding in water, summarized as follows.

We described the nature of the x-ray absorption process and the importance of the dipole approximation, the one-center approximation and the Franck-Condon principle and their implications to the water XAS spectrum. The $\mathrm{C}_{2 \mathrm{v}}$ symmetry in gas-phase water gives rise to significant $2 p$ character and strong intensity for the LUMO orbital $\left(4 \mathrm{a}_{1}\right)$ as opposed to the symmetric situation in $\mathrm{CH}_{4}$. Differences in the initial and final state potential energy curves lead to broad spectral lines as seen in the $4 \mathrm{a}_{1}$ and $2 \mathrm{~b}_{2}$ peaks of gas phase water, whereas excitation into the non-bonding Rydberg states results in sharp features. The disappearance, upon condensation, of the features associated with the free molecule unoccupied molecular orbitals is the result of a strong rehybridization of the unoccupied molecular orbitals due to their large spatial extent relative to the short distance between water molecules in the condensed phase resulting from the formation of H-bonds. In particular, the LUMO is almost completely suppressed in the near-tetrahedral H-bond symmetry of ice, and we form an intense post-edge peak that is directly associated with states formed along H-bonds. The delocalized nature of the post-edge resonance is directly derived from resonant Auger and x-ray emission spectroscopies and its shape and position can be understood by the "bond-length-with-a-ruler" concept in which the energy of the anti-bonding band reflects the H-bond distance, resulting in, e.g., a significant broadening in the amorphous ice phase and a shift to higher energies due to shorter H-bonds in spectra for the Eigen form of protonated water. When the symmetry of the H-bond network is broken, e.g., at the surface of ice and in defects in the water overlayer on a metal surface, intensity is shifted from the post-edge to lower energies where a distinct pre-edge and a mainedge are formed that are different from the gas-phase. From ammonia termination of the ice surface, from adsorbates and ice films on metal surfaces we can unambiguously associate the pre-edge with water molecules having an asymmetric H-bond situation in which one of the donor sides is $\mathrm{H}$-bonded and the other side has a dangling $\mathrm{OH}$. Model 2-D systems and resonant Auger, as well as DFT calculations, furthermore show that the pre-edge corresponds to a strongly localized state along the dangling $\mathrm{OH}$.

We discussed the fact that spectra of ice, as obtained from thin films grown on single-crystal surfaces, are more complicated than previously anticipated due to heterogeneities in the structure. It was proposed that various forms of high density amorphous ice could co-exist with crystalline cubic ice and low density amorphous ice in the ice films giving rise to variable intensities of the pre-edge and main-edge sharp spectral features depending on the preparation conditions of the ice films. It will be essential to develop single-crystal ice model systems which are both fully homogeneous and for which the structure is well characterized in order to finally determine the detailed cubic ice spectrum.

The most reliable manner to measure the liquid water x-ray absorption spectrum is by using transmission with a sample area small enough that the thickness is constant or by x-ray Raman scattering at low momentum transfer. It is clear that the x-ray absorption process provides information regarding the $\mathrm{H}$-bonding network in water with some unique, sharp spectral features. The pre-edge and main-edge intensities show an increase and the post-edge a decrease in intensity with increasing temperature. There is also a shift of the pre-edge and main-edge energy 
positions towards the gas phase value with increasing temperature. Bringing in also recent SAXS and XES results these observations can be understood in a picture based on that water is inhomogeneous with a fluctuating $\mathrm{H}$-bonding network around two structures, strongly tetrahedral and H-bond distorted. The two types of local structures are driven by incommensurate requirements for minimizing enthalpy (tetrahedral) and maximizing entropy (H-bonds distorted). We can consider that these structures are related to high density liquid (HDL) water and low density liquid (LDL) water, as proposed by Stanley and coworkers (133). The HDL structure dominates in the liquid at ambient conditions. We change the balance slightly towards LDL when we increase the mass of protons towards $\mathrm{D}_{2} \mathrm{O}$, but the spectra indicate additional quantum effects beyond a simple comparison to a temperature change. If we put $\mathrm{NaCl}$ salt in water the spectral changes are similar to a temperature increase, but without any shifts in the positions of the spectral features. This can be understood if the effect of adding salt is similar to an increase in pressure which converts water towards higher concentrations of HDL.

Determining the detailed local structures of the HDL and LDL species will require theoretical simulations. At this point no theoretical spectrum calculations based on structures from MD simulations have been shown to reproduce the intensity and the absolute energy position of the pre-edge, main-edge and post-edge features. Using the half-core-hole or transition-potential approximation, which so far theoretically best describes the ice spectrum (28), the computed spectra, based on structures from MD simulations of the liquid, mainly show an intense post-edge at an energy position intermediate between the main-edge and the post-edge in ice, see fig 41. Following the bond length with a ruler concept this would then be consistent with small $\mathrm{H}$-bond distortions from the tetrahedral coordination in ice resulting in a downward

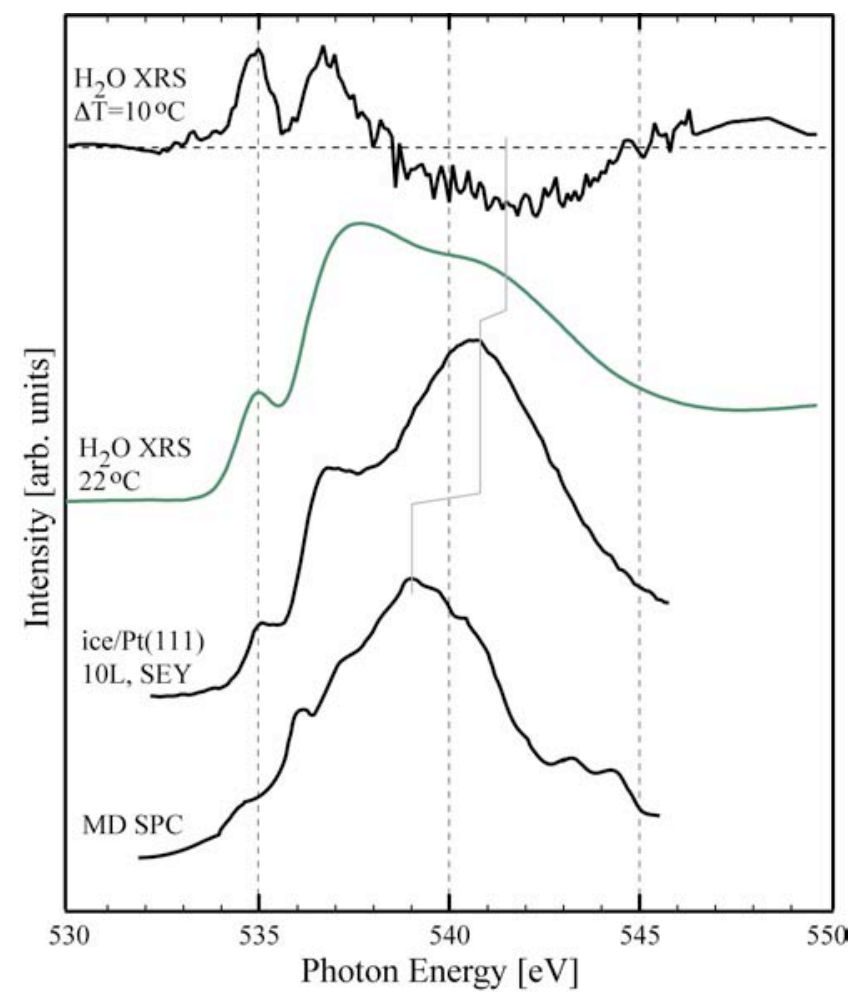

Fig. 41. Comparison between experimental XRS temperature difference and XAS spectra of water (3) with theoretical spectrum calculations of a sum of all configurations in an SPC MD dump (6). 
shift in the post-edge position. On the contrary the experimental post-edge position in water is nearly the same as in ice (small shift to higher energies), which indicates that the tetrahedral structures contain shorter distances than in the simulations. This directly indicates that the tetrahedral structures in water are locally similar to those in ice. Fig. 41 also shows, in the spectrum based on MD structures, small pre-edge intensity reflecting only a minor contribution of strongly distorted H-bonded structures. The question is if the MD simulations give structures which are more similar to an average between the two fluctuating structures in water. This would be consistent with the $\mathrm{O}-\mathrm{O}$ pair correlation function obtained from x-ray diffraction where the first peak is of lower height and broader than the pair correlations derived from MD simulations (5; 39). The liquid has a much broader range of structures with a larger variation in the H-bond length due to the appearance of the bimodal distribution of local structures than the neartetrahedral structures as obtained from many of the popular MD simulations. However, to fully understand the structure of water including its dynamics will require a combination of many different experimental techniques together with various theoretical simulations.

\section{Acknowledgement}

Supported by the National Science Foundation (US) CHE-0809324 and CHE-0431425 and the Swedish Research Council. Portions of this research were carried out at the Stanford Synchrotron Radiation Lightsource (SSRL), a national user facility operated by Stanford University on behalf of the U.S. Department of Energy, Office of Basic Energy Sciences.

\section{References}

[1] W.C. Röntgen, Ann. Phys. 45 (1892) 91.

[2] J.D. Bernal, R.H. Fowler, J. Chem. Phys. 1 (1933) 515.

[3] Ph. Wernet, D. Nordlund, U. Bergmann, M. Cavalleri, M. Odelius, H. Ogasawara, L.Å. Näslund, T.K. Hirsch, L. Ojamäe, P. Glatzel, L.G.M. Pettersson, A. Nilsson, Science 304 (2004) 995.

[4] M. Leetmaa, M. Ljungberg, H. Ogasawara, M. Odelius, L.Å. Näslund, A. Nilsson, L.G.M. Pettersson, J. Chem. Phys. 125 (2006) 244510.

[5] M. Leetmaa, K.T. Wikfeldt, M.P. Ljungberg, M. Odelius, J. Swenson, A. Nilsson, L.G.M. Pettersson, J. Chem. Phys. 129 (2008) 084502.

[6] M. Odelius, M. Cavalleri, A. Nilsson, L.G.M. Pettersson, Phys. Rev. B 73 (2006) 024205.

[7] A.K. Soper, J. Phys.:Condens. Matter 17 (2005) S3273.

[8] G. Brancato, R. Nadia, V. Barone, Phys. Rev. Lett. 100 (2008) 107401.

[9] M. Cavalleri, M. Odelius, A. Nilsson, L.G.M. Pettersson, J. Chem. Phys. 121 (2004) 10065.

[10] M. Cavalleri, M. Odelius, D. Nordlund, A. Nilsson, L.G.M. Pettersson, Phys. Chem. Chem. Phys. 7 (2005) 2854.

[11] M. Cavalleri, H. Ogasawara, L.G.M. Pettersson, A. Nilsson, Chem. Phys. Lett. 364 (2002) 363.

[12] M.V. Fernandez-Serra, E. Artacho, Phys. Rev. Lett. 96 (2006) 016404.

[13] O. Fuchs, M. Zharnikov, L. Weinhardt, M. Blum, M. Weigand, Y. Zubavichus, M. Bär, F. Maier, J.D. Denlinger, C. Heske, M. Grunze, E. Umbach, Phys. Rev. Lett. 100 (2008) 027801. 
[14] J.H. Guo, Y. Luo, A. Augustsson, J.E. Rubensson, C. Såthe, H. Ågren, H. Siegbahn, J. Nordgren, Phys. Rev. Lett 89 (2002) 137402.

[15] B. Hetényi, F. De Angelis, P. Giannozzi, R. Car, J. Chem. Phys. 120 (2004) 8632-8637.

[16] M. Iannuzzi, J. Chem. Phys. 128 (2008) 204506.

[17] S. Kashtanov, A. Augustsson, Y. Luo, J.H. Guo, C. Såthe, J.E. Rubensson, H. Siegbahn, J. Nordgren, H. Ågren, Phys. Rev. B 69 (2004) 024201.

[18] S. Myneni, Y. Luo, L.Å. Näslund, M. Cavalleri, L. Ojamäe, H. Ogasawara, A. Pelmenschikov, Ph. Wernet, P. Väterlein, C. Heske, Z. Hussain, L.G.M. Pettersson, A. Nilsson, J. Phys.:Condens. Matter 14 (2002) L213-L219.

[19] A. Nilsson, Ph. Wernet, D. Nordlund, U. Bergmann, M. Cavalleri, M. Odelius, H. Ogasawara, L.-Å. Näslund, T.K. Hirsch, L. Ojamäe, P. Glatzel, L.G.M. Pettersson, Science 308 (2005) 793a.

[20] D. Nordlund, H. Ogasawara, H. Bluhm, O. Takahashi, M. Odelius, M. Nagasono, L.G.M. Pettersson, A. Nilsson, Phys. Rev. Lett. 99 (2007) 217406.

[21] D. Prendergast, G. Galli, Phys. Rev. Lett. 96 (2006) 215502.

[22] J.D. Smith, C.D. Cappa, B.M. Messer, R.C. Cohen, R.J. Saykally, Science 308 (2005) $793 b$.

[23] J.D. Smith, C.D. Cappa, B.M. Messer, W.S. Drisdell, R.C. Cohen, R.J. Saykally, J. Phys. Chem. B 110 (2006) 20038-20045.

[24] J.D. Smith, C.D. Cappa, K.R. Wilson, B.M. Messer, R.C. Cohen, R.J. Saykally, Science 306 (2004) 851-853.

[25] T. Tokushima, Y. Harada, O. Takahashi, Y. Senba, H. Ohashi, L.G.M. Pettersson, A. Nilsson, S. Shin, Chem. Phys. Lett. 460 (2008) 387.

[26] J.S. Tse, D.M. Shaw, D.D. Klug, S. Patchkovski, G. Vanko, G. Monaco, M. Krisch, Phys. Rev. Lett. 100 (2008) 095502.

[27] R.L.C. Wang, H.J. Kreuzer, M. Grunze, Phys. Chem. Chem. Phys. 8 (2006) 4744.

[28] M. Leetmaa, M.P. Ljungberg, A. Lyubartsev, A. Nilsson, L.G.M. Pettersson, submitted to J. El. Spec. Rel. Phenom. (2009).

[29] G.E. Walrafen, J. Chem. Phys. 120 (2004) 4868.

[30] D. Nordlund, H. Ogasawara, Ph. Wernet, M. Nyberg, M. Odelius, L.G.M. Pettersson, A. Nilsson, Chem. Phys. Lett. 395 (2004) 161.

[31] P. Parent, C. Laffon, C. Mangeney, F. Bournel, M. Tronc, J. Chem. Phys. 117 (2002) 10842.

[32] U. Bergmann, Ph. Wernet, P. Glatzel, M. Cavalleri, L.G.M. Pettersson, A. Nilsson, S.P. Cramer, Phys. Rev. B 66 (2002) 092107.

[33] B. Winter, U. Hergenhahn, M. Faubel, O. Björneholm, I.V. Hertel, J. Chem. Phys. 127 (2007) 094501.

[34] A. Nilsson, L.G.M. Pettersson, Surf. Sci. Reps. 55 (2004) 49.

[35] M. Odelius, J. Phys. Chem. A 113 (2009) 8176.

[36] M. Odelius, Phys. Rev. B 79 (2009) 144204.

[37] T. Tokushima, Y. Harada, Y. Horikawa, O. Takahashi, Y. Senba, H. Ohashi, L.G.M. Pettersson, A. Nilsson, S. Shin, submitted to J. El. Spec. Rel. Phenom. (2009).

[38] C. Huang, K.T. Wikfeldt, T. Tokushima, D. Nordlund, Y. Harada, U. Bergmann, M. Niebur, T. Weiss, Y. Horikawa, M. Leetmaa, M.P. Ljungberg, O. Takahashi, A. Lenz, L. Ojamäe, A.P. Lyubartsev, S. Shin, L.G.M. Pettersson, A. Nilsson, Proc. National Acad. Sc. (USA) 106 (2009) 15214. 
[39] K.T. Wikfeldt, M. Leetmaa, M.P. Ljungberg, A. Nilsson, L.G.M. Pettersson, J. Phys. Chem. B 113 (2009) 6246.

[40] J. Stöhr, NEXAFS spectroscopy, Berlin ; New York, Springer-Verlag, 1992.

[41] B.X. Yang, J. Kirz, Phys. Rev. B 36 (1987) 1361.

[42] D.T. Bowron, M.H. Krisch, A.C. Barnes, J.L. Finney, A. Kaprolat, M. Lorenzen, Phys. Rev. B 62 (2000) R9223.

[43] L.Å. Näslund, J. Lüning, Y. Ufuktepe, H. Ogasawara, Ph. Wernet, U. Bergmann, L.G.M. Pettersson, A. Nilsson, J. Phys. Chem. B 109 (2005) 13835.

[44] The standard transmission data were obtained using BL 10-1 at SSRL and the SXTM transmission measurements using BL 11 at the ALS. Both measurements were conducted with an energy resolution of $0.1 \mathrm{eV}$.

[45] R. Carboni, S. Giovannini, G. Antonioli, F. Boscherini, Phys. Scr. T115 (2005) 986.

[46] S. Eisebitt, T. Boske, J.E. Rubensson, W. Eberhardt, Phys. Rev. B 47 (1993) 14103.

[47] R. Nakajima, J. Stöhr, Y.U. Idzerda, Phys. Rev. B 59 (1999) 6421.

[48] L.Å. Näslund, D.C. Edwards, Ph. Wernet, U. Bergmann, H. Ogasawara, L.G.M.

Pettersson, S. Myneni, A. Nilsson, J. Phys. Chem. A 109 (2005) 5995.

[49] T.J. Regan, H. Ohldag, C. Stamm, F. Nolting, J. Luning, J. Stohr, R.L. White, Phys. Rev. B 64 (2001) 214422.

[50] H. Bluhm, D.F. Ogletree, C.S. Fadley, Z. Hussain, N. Salmeron, J. Phys.;Condensed Matter 14 (2002) L227.

[51] N. Timneanu, C. Caleman, J. Hajdu, D.v.d. Spoel, Chem. Phys. Chem. 299 (2004) 277.

[52] A. Nilsson, E. Zdansky, H. Tillborg, O. Björneholm, N. Mårtensson, J.N. Andersen, R. Nyholm, Chem. Phys. Lett. 197 (1992) 12.

[53] Y. Mizuno, Y. Ohmura, J. Phys. Soc. Japan 22 (1967) 445.

[54] T. Suzuki, J. Phys. Soc. Japan 22 (1967) 1139.

[55] U. Bergmann, P. Glatzel, S.P. Cramer, Microchemical Journal 71 (2002) 221.

[56] M. Krisch, F. Sette, Surf. Rev. Lett. 9 (2002) 969.

[57] T.T. Fister, K.P. Nagle, F.D. Vila, G.T. Seidler, C. Hamner, J.O. Cross, J.J. Rehr, Phys. Rev. B 79 (2009) 174117.

[58] U. Bergmann, A. Di Cicco, Ph. Wernet, E. Principi, P. Glatzel, A. Nilsson, J. Chem. Phys. 127 (2007) 174504.

[59] U. Bergmann, D. Nordlund, Ph. Wernet, M. Odelius, L.G.M. Pettersson, A. Nilsson, Phys. Rev. B 76 (2007) 024202

[60] Y.Q. Cai, H.K. Mao, P.C. Chow, J.S. Tse, Y. Ma, S. Patchkovskii, J.F. Shu, V. Struzhkin, R.J. Hemley, H. Ishii, C.C. Chen, I. Jarrige, C.T. Chen, S.R. Shieh, E.P. Huang, C.C. Kao, Phys. Rev. Lett. 94 (2005) 025502.

[61] H. Fukui, S. Huotari, D. Andrault, T. Kawamoto, J Chem. Phys. 127 (2007) 134502.

[62] W.L. Mao, H.K. Mao, Y. Meng, P.J. Eng, M.Y. Hu, P. Chow, Y.Q. Cai, J.F. Shu, R.J. Hemley, Science 314 (2006) 636.

[63] I. Waluyo, D. Nordlund, U. Bergmann, L.G.M. Pettersson, A. Nilsson, J Chem. Phys. 131 (2009) 031103.

[64] Ph. Wernet, D. Testemale, J.-L. Hazemann, R. Argoud, P. Glatzel, L.G.M. Pettersson, A. Nilsson, U. Bergmann, J. Chem. Phys. 123 (2005) 154503.

[65] M.H. Krisch, F. Sette, C. Masciovecchio, R. Verbeni, Phys. Rev. Lett. 78 (1997) 2843.

[66] D. Nordlund, M. Odelius, H. Bluhm, H. Ogasawara, L.G.M. Pettersson, A. Nilsson, Chem. Phys. Lett 460 (2008) 86. 
[67] B. Winter, R. Weber, W. Widdra, M. Dittmar, M. Faubel, I.V. Hertel, J. Phys. Chem. A 108 (2004) 2625.

[68] R. Sankari, M. Ehara, H. Nakatsuji, Y. Senba, K. Hosokawa, H. Yoshida, A. De Fanis, Y. Tamenori, S. Aksela, K. Ueda, Chem. Phys. Lett. 380 (2003) 647.

[69] I. Hjelte, M.N. Piancastelli, R.F. Fink, O. Björneholm, M. Bässler, R. Feifel, A. Giertz, H. Wang, K. Wiesner, A. Ausmees, C. Miron, S.L. Sorensen, S. Svensson, Chem. Phys. Lett. 334 (2001) 151.

[70] A. Naves de Brito, R. Feifel, A. Mocellin, A.B. Machado, S. Sundin, I. Hjelte, S.L. Sorensen, O. Björneholm, Chem. Phys. Lett. 309 (1999) 377.

[71] O. Takahashi, M. Odelius, D. Nordlund, A. Nilsson, H. Bluhm, L.G.M. Pettersson, J. Chem. Phys. 124 (2006) 064307.

[72] A. Hiraya, K. Nobusada, M. Simon, K. Okada, T. Tokushima, Y. Senba, H. Yoshida, K. Kamimori, H. Okumura, Y. Shimizu, A.-L. Thomas, P. Millie, I. Koyano, K. Ueda, Phys. Rev. A 63 (2001) 042705.

[73] E.T.J. Nibbering, T. Elsaesser, Chem. Rev. 104 (2004) 1887.

[74] J. Schirmer, A.B. Trofimov, K.J. Randall, J. Feldhaus, A.M. Bradshaw, Y. Ma, C.T. Chen, F. Sette, Phys. Rev. A 47 (1993) 1136.

[75] The multilayer $\mathrm{H}_{2} \mathrm{O}$ and $\mathrm{CH}_{4}$ ice XAS spectra were measured at BL I511 at MAX-lab, Sweden. The SX700 monochromator was operated at around $0.1 \mathrm{eV}$ resolution with a c value of 1.8 to suppress second-order contributions. Details about the $\mathrm{H}_{2} \mathrm{O}$ ice preparation and acquisition can be found in ref [30]. A multilayer film of $\mathrm{CH}_{4}$ was adsorbed below 35 $\mathrm{K}$ on $\mathrm{Pt}(111)$ using a pulsed gas-delivery system. Coverage and cleanliness was checked using XPS, in particular to avoid CO contamination. The XAS spectra were recorded in AEY, using a $7 \mathrm{eV}$ wide energy window centered around the C KLL Auger line at 262 $\mathrm{eV}$ (using a SCIENTA hemispherical analyzer with pass energy $300 \mathrm{eV}$ and $0.8 \mathrm{~mm}$ slits with the electron analyzer oriented perpendicular to the E-vector of the incoming photons in order to suppress direct photoemission peaks.

[76] T. Moeller, Inorganic chemistry, New York, John Wiley \& Sons Inc., 1953.

[77] S. Hufner, Photoelectron Spectroscopy, Berlin-Heidelberg, Springer-Verlag, 1995.

[78] M. Neeb, J.E. Rubensson, M. Biermann, W. Eberhardt, J. El. Spec. Rel. Phenom. 67 (1994) 261.

[79] O. Björneholm, A. Nilsson, A. Sandell, B. Hernnäs, N. Mårtensson, Phys. Rev. Lett. 68 ( 1992) 1892.

[80] O. Björneholm, S. Sundin, S. Svensson, R.R.T. Marinho, A.N. deBrito, F. Gel'mukhanov, H. Ågren, Phys. Rev. Lett 79 (1997) 3150.

[81] P.A. Brühwiler, O. Karis, N. Mårtensson, Rev. Mod. Phys. 74 (2002) 703.

[82] A. Föhlisch, P. Feulner, F. Hennies, A. Fink, D. Menzel, D. Sanchez-Portal, P.M. Echenique, W. Wurth, Nature 436 (2005) 373.

[83] F. Gel'mukhanov, H. Ågren, Phys. Reps. 312 (1999) 87.

[84] O. Karis, A. Nilsson, M. Weinelt, T. Wiell, C. Puglia, N. Wassdahl, N. Mårtensson, M. Samant, J. Stöhr, Phys. Rev. Lett 76 (1996) 1380.

[85] A. Nilsson, J. El. Spec. Rel. Phenom. 126 (2002) 3.

[86] A. Sandell, O. Björneholm, A. Nilsson, B. Hernnäs, J.N. Andersen, N. Mårtensson, Phys. Rev. B 49 (1994) 10136.

[87] A. Sandell, O. Hjortstam, A. Nilsson, P.A. Brühwiler, O. Eriksson, P. Bennich, P. Rudolf, J.M. Wills, B.Johansson, N. Mårtensson, Phys. Rev. Lett. 78 (1997) 4994. 
[88] J. Schnadt, P.A. Brüwhiler, L. Patthey, J.N. O'Shea, S. Södergren, M. Odelius, R. Ahuja, O. Karis, M. Bässler, P. Persson, H. Siegbahn, S. Lunell, N. Mårtensson, Nature 418 (2002) 620.

[89] A. Nilsson, N. Wassdahl, M. Weinelt, O. Karis, T. Wiell, P. Bennich, J. Hasselström, A. Föhlisch, J. Stöhr, M. Samant, Appl. Phys. A 65 (1997) 147.

[90] F. Sette, J. Stöhr, A.P. Hitchcook, Chem. Phys. Lett. 110 (1984) 517.

[91] J. Stöhr, J.L. Gland, W. Eberhardt, D. Outka, R.J. Madix, F. Sette, R.J. Koestner, U. Doebler, Phys. Rev. Lett. 51 (1983) 2414.

[92] J. Stöhr, F. Sette, A.L. Johnson, Phys. Rev. Lett. 53 (1984) 1684.

[93] C. Puglia, Adsorption of oxygen and nitrogen on platinum and alkali modified graphite, PhD Thesis, Uppsala University, 1997.

[94] C. Puglia, A. Nilsson, B. Hernnäs, O. Karis, P. Bennich, N. Mårtensson, Surf. Sci. 342 (1995) 171.

[95] A. Nilsson, H. Ogasawara, M. Cavalleri, D. Nordlund, M. Nyberg, Ph. Wernet, L.G.M. Pettersson, J. Chem. Phys. 122 (2005) 154505.

[96] M. Cavalleri, L.Å. Näslund, D.C. Edwards, Ph. Wernet, H. Ogasawara, S. Myneni, L. Ojamäe, M. Odelius, A. Nilsson, L.G.M. Pettersson, J. Chem. Phys. 124 (2006) 194508.

[97] G.A. Kimmel, N.G. Petrik, Z. Dohnalek, B.D. Kay, Phys. Rev. Lett. 95 (2005) 166102.

[98] I. Waluyo, D. Nordlund, L.Å. Näslund, H. Ogasawara, L.G.M. Pettersson, A. Nilsson, Surf. Sci. 602 (2008) 2004.

[99] G. Zimbitas, S. Haq, A. Hodgson, J. Chem. Phys. 123 (2005) 17470.

[100] J.E. Schaff, J.T. Roberts, J. Phys. Chem. 100 (1996) 1415.

[101] Z. Dohnalek, R.L. Ciolli, G.A. Kimmel, K.P. Stevenson, R.S. Smith, B.D. Kay, J. Chem. Phys. 110 (1999) 548.

[102] P. Löfgren, P. Ahlström, D.V. Chakarov, J. Lausmaa, B. Kasemo, Surf. Sci. 367 (1996) L19.

[103] The experiments were performed at beamline 5-1 at SSRL using Auger yield detection with an energy resolution of $0.1 \mathrm{eV}$. The amorphous $\mathrm{D}_{2} \mathrm{O}$ ice films of around 80 layers were grown on the clean $\mathrm{Pt}(111)$ surface at $100 \mathrm{~K}$ at a deposition rate of 0.08 layers/s using a pulsed gas delivery system. Crystalline ice was obtained by isothermal heating at $150 \mathrm{~K}$ of the amorphous film and changes in the water desorption rate were monitored through mass spectrometry.

[104] Y. Zubavichus, M. Zharnikov, Y.J. Yang, O. Fuchs, E. Umbach, C. Heske, M. Grunze, Langmuir 22 (2006) 7241.

[105] The amorphous ice sample was prepared by condensing deionized water in ultrahigh vacuum onto a $\mathrm{Si}_{3} \mathrm{~N}_{4}$ membrane that was cooled to liquid Nitrogen temperature. The crystalline ice Ih sample was made from the amorphous sample by slowly heating it to about $150 \mathrm{~K}$. Crystallization was observed to be complete after approximately $30 \mathrm{~min}$. Both ice samples were approximately $200 \mathrm{~nm}$ thick.

[106] IR spectra were recorded in the reflection-absorption geometry using a Fourier transform infrared spectrometer (Bruker Vector 33), a mirror system and a narrow band mercury cadmium telluride (MCT) detector. A collimated IR beam was focused on the sample at an incident angle of $80^{\circ}$ by a $90^{\circ}$ off-axis parabolic mirror through a $\mathrm{BaF}_{2}$ window. The reflected beam from the sample was refocused on the detector by a pair of $90^{\circ}$ off-axis parabolic mirrors. A clean surface served as a background reference for the absorption spectrum. A resolution of $4 \mathrm{~cm}^{-1}$ or $8 \mathrm{~cm}^{-1}$ and p-polarized light were used for IRAS 
measurement. Notably, no absorption peak of the ice film was observed in s-polarized IRAS due to the surface dipole selection rule for metal surfaces.

[107] B. Rowland, P.J. Devlin, J. Chem. Phys. 94 (1991) 812.

[108] D. Coulman, A. Puschmann, U. Höfer, H.P. Steinruck, W. Wurth, P. Feulner, D. Menzel, J. Chem. Phys. 93 (1990) 58.

[109] R.A. Rosenberg, P.R. Laroe, V. Rehn, J. Stöhr, R. Jäger, C.C. Parks, Phys. Rev. B 28 (1983) 3026.

[110] P. Jenniskens, D.F. Blake, Science 265 (1994) 753.

[111] A.H. Narten, C.G. Venkatesh, S.A. Ruice, J. Chem. Phys. 64 (1976) 1106.

[112] $\mathrm{BaF}_{2}$ (111) single crystals were freshly cleaved and placed in the UHV chamber without prolonged exposure to air. Amorphous ice layers were prepared by dosing water vapor on the $\mathrm{BaF}_{2}(111)$ surface at $100 \mathrm{~K}$. A monolayer is assumed to be equivalent to the amount of a single layer of water on $\mathrm{Ni}(111)$ as calibrated by XPS and where the same dosage procedure was applied. Crystalline ice layers were prepared by annealing amorphous layers to the indicated temperatures. X-ray absorption spectra were recorded in PEY mode with a resolution of $0.1 \mathrm{eV}$.

[113] M.M. Koza, B. Geil, K. Winkel, C. Kähler, F. Czeschka, M. Scheuermann, H. Schober, T. Hansen, Phys. Rev. Lett. 94 (2005) 125506.

[114] M.M. Koza, T. Hansen, R.P. May, H. Schober, J. Non-Cryst. Solids 352 (2006) 4988.

[115] H. Schober, M. Koza, A. Tölle, F. Fujara, C.A. Angell, R. Böhmer, Physica B 241-243 (1998) 897.

[116] C.A. Tulk, C.J. Benmore, J. Utquidi, D.D. Klug, J. Neuefeind, B. Tomberli, P.A. Egelstaff, Science 297 (2002) 1320.

[117] H. Ogasawara, B. Brena, D. Nordlund, M. Nyberg, A. Pelmenschikov, L.G.M. Pettersson, A. Nilsson, Phys. Rev. Lett. 89 (2002) 27610.

[118] D. Nordlund, H. Ogasawara, K.J. Andersson, M. Tatarkhanov, M. Salmerón, L.G.M. Pettersson, A. Nilsson, submitted.

[119] K. Andersson, A. Nikitin, L.G.M. Pettersson, A. Nilsson, H. Ogasawara, Phys. Rev. Lett. 93 (2004) 196101.

[120] A. Hodgson, S. Haq, Surf. Sci. Reps. Doi:10.1016/j.surfrep.2009.07.001 (2009).

[121] M. Gallagher, A. Omer, G.R. Darling, A. Hodgson, Faraday Disc. Chem. Soc. 141 (2009) 231.

[122] T. Kondo, S. Mae, H.S. Kato, M. Kawai, Surf. Sci. 600 (2006) 3570.

[123] T. Schiros, L.Å. Näslund, K. Andersson, J. Gyllenpalm, G.S. Karlberg, M. Odelius, H. Ogasawara, L.G.M. Pettersson, A. Nilsson, J. Phys. Chem. C 111 (2007) 15003.

[124] The co-adsorbed layer was prepared through a heat ramp from $\mathrm{T}<100 \mathrm{~K}$ to $156 \mathrm{~K}$ of a multilayer $\mathrm{D}_{2} \mathrm{O}$ film on top of a $(2 \times 2)-\mathrm{O}$ layer. A full coverage of the $\mathrm{D}_{2} \mathrm{O}+\mathrm{OD}$ layer was obtained when the (2x2)-O coverage was 0.23 ML. At 0.09 ML (2x2)-O, however, the obtained coverage was $0.67 \mathrm{ML}$ and by extending the heat ramp to $166 \mathrm{~K}$ the obtained coverage became $0.58 \mathrm{ML}$.

[125] J. Hasselström, O. Karis, M. Weinelt, N. Wassdahl, A. Nilsson, M. Nyberg, L.G.M. Pettersson, M. Samant, J. Stöhr, Surf. Sci. 407 (1998) 221.

[126] M. Nyberg, M. Odelius, A. Nilsson, L.G.M. Pettersson, J. Chem. Phys. 119 (2003) 12577.

[127] S. Barlow, K. Kitching, S. Haq, N.V. Richardsson, Surf. Sci. 401 (1998) 322. 
[128] N.A. Booth, D.P. Woodruff, O. Schaff, T. Giessel, R. Lindsay, P. Baumgärtel, A.M. Bradshaw, Surf. Sci. 397 (1998) 258.

[129] R.W. Hendrick, P.G. Mardon, L.B. Shaffer, J. Chem. Phys. 61 (1974) 319.

[130] C.A. Angell, Science 319 (2008) 582.

[131] I. Brovchenko, A. Oleinikova, Chem. Phys. Chem. 9 (2008) 2660.

[132] P.G. Debenedetti, J. Phys.: Condens. Matter 15 (2003) R1669.

[133] O. Mishima, H.E. Stanley, Nature 396 (1998) 329.

[134] P.H. Poole, F. Sciortino, U. Essmann, H.E. Stanley, Nature 360 (1992) 324.

[135] P. Kumar, S.V. Buldyrev, S.R. Becker, P.H. Poole, F.W. Starr, H.E. Stanley, Proc. National Acad. of Sci. (USA) 104 (2007) 9575.

[136] L. Xu, P. Kumar, S.V. Buldyrev, S.-H. Chen, P.H. Poole, F. Sciortino, H.E. Stanley, Proc. National Acad. Sci. (USA) 102 (2005) 16558.

[137] A.V. Okhulkov, Y.N. Demianets, Y.E. Gorbaty, J. Chem. Phys. 100 (1994) 1578.

[138] A.K. Soper, M.A. Ricci, Phys. Rev. Lett. 84 (2000) 2881.

[139] J. Urquidi, S. Singh, C.H. Cho, G.W. Robinson, Phys. Rev. Lett. 83 (1999) 2348.

[140] L. Fu, X-ray study of the structure of liquid water, PhD Thesis, Stanford University, 2007.

[141] A.H. Narten, H.A. Levy, Science 165 (1969) 447.

[142] A.H. Narten, H.A. Levy, J. Chem. Phys. 55 (1971) 2263.

[143] G. Némethy, H.A. Scheraga, J. Chem. Phys. 41 (1964) 680.

[144] Y.S. Badyal, D.L. Price, M.L. Saboungi, D.R. Haeffner, S.D. Shastri, J. Chem. Phys. 116 (2002) 10833.

[145] C.H. Cho, J. Urquidi, S. Singh, G.W. Robinson, J. Phys. Chem. B 103 (1999) 1991.

[146] P.A. Egelstaff, Phys. Chem. Liq. 40 (2002) 203.

[147] R.T. Hart, C.J. Benmore, J. Neuefeind, S. Kohara, B. Tomberli, P.A. Egelstaff, Phys. Rev. Lett. 94 (2005) 047801.

[148] J.H. Root, P.A. Egelstaff, A. Hime, Chem, Phys. 109 (1986) 437.

[149] A.K. Soper, C.J. Benmore, Phys. Rev. Lett. 101 (2008) 065502.

[150] B. Tomberli, C.J. Benmore, P.A. Egelstaff, J. Neuefeind, V. Honkimäki, J. Phys. Condens. Matter 12 (2000) 2597.

[151] M. Vedamuthu, S. Singh, G.W. Robinson, J. Phys. Chem. 98 (1994) 2222.

[152] M. Odelius, H. Ogasawara, D. Nordlund, O. Fuchs, L. Weinhardt, F. Maier, E. Umbach, C. Heske, Y. Zubavichus, M. Grunze, J.D. Denlinger, L.G.M. Pettersson, A. Nilsson, Phys. Rev. Lett. 94 (2005).

[153] M.F. Fox, E. Hayon, J. Phys. Chem. 76 (1972) 2703.

[154] D.M. Bartels, R.A. Crowell, J. Phys. Chem. 104 (2000) 3349.

[155] W.H. Shaffer, R.P. Shuman, J. Chem. Phys. 12 (1944) 504.

[156] J.R. Houlston, J. Chem. Phys. 50 (1969) 1483.

[157] C.D. Cappa, J.D. Smith, K.R. Wilson, B.M. Messer, M.K. Gilles, R.C. Cohen, R.J. Saykally, J. Phys. Chem. B 109 (2005) 7046.

[158] R. Leberman, A.K. Soper, Nature 378 (1995) 364. 